\title{
El viaducto del río Ulla en el Eje Atlántico de Alta Velocidad: una celosía mixta récord del mundo
}

\author{
Viaduct over Ulla River in the Atlantic High Speed Railway Line: \\ A composite (steel-concrete) truss world record \\ Francisco Millanes Mato , Miguel Ortega Cornejo y Rubén A. Estévez Sánchez
}

\begin{abstract}
Resumen
Con la puesta en servicio del Eje Atlántico de Alta Velocidad entre Pontevedra y A Coruña, en la primavera de 2015, el viaducto sobre el río Ulla se ha convertido en el récord del mundo en la tipología de celosía mixta de alta velocidad con tres vanos de $225+240+225 \mathrm{~m}$ que superan al del puente de Nantenbach sobre el río Main, en Alemania, que ostentaba el récord desde su conclusión en 1993 con $208 \mathrm{~m}$ de luz.

Este artículo describe con detalle la concepción y las principales particularidades del viaducto, el novedoso sistema de control de calidad de la ejecución implementado con gran éxito, así como los complejos y singulares procesos constructivos empleados para la ejecución del tablero y de las cimentaciones de las pilas en el cauce, junto con los excepcionales medios auxiliares que ha sido necesario fabricar ex profeso para su ejecución.
\end{abstract}

Palabras clave: Estructura metálica; Celosía; Doble acción mixta; Avance en voladizos; Izados; Lanzamiento

\section{Abstract}

With the commissioning of the Atlantic High Speed Railway Line between Pontevedra and A Coruna, in the spring of 2015, the viaduct over the river Ulla has achieved the world record in the typology of high speed composite truss bridge, with three main spans of $225+240+225 \mathrm{~m}$. This exceeds the Nantenbach Bridge over Main River in Germany, which held the record since its conclusion in 1993 with a $208 \mathrm{~m}$ span.

This article describes in detail the design and the main features of the viaduct, the new system of quality control of the execution implemented with great success. A description is also presented on the complex and outstanding construction processes used for the execution of the deck of the bridge and the foundation of the piers located on the river, with the exceptional temporary works that have been fabricated ex profeso for the execution. 


\section{Introducción}

El viaducto sobre el río Ulla, en la desembocadura de la ría de Arosa, constituye la actuación de mayor alcance del Eje Atlántico de Alta Velocidad entre Pontevedra y A Coruña, y tras su conclusión y puesta en servicio se ha convertido en el viaducto mixto en celosía de alta velocidad con los 3 vanos centrales de mayor luz del mundo [1].

Su ubicación, próxima al estuario de la ría de Arosa, en un entorno natural de extraordinaria belleza y sometido a especiales condicionantes medioambientales, aconsejó a la Dirección General de Ferrocarriles la convocatoria de un concurso restringido de ideas. La solución presentada por IDEAM resultó la ganadora y se desarrolló en el proyecto constructivo, orientando la solución a resolver la siguiente problemática:

- Cuidar la estética de la solución y su integración medioambiental en el entorno.

- Reducir el número de pilas en el cauce, siempre dentro de la viabilidad de los viaductos de alta velocidad y de unos costes de ejecución asumibles.

- Plantear procesos constructivos lo más independientes del cauce y de sus riberas.

- Buscar la máxima transparencia y mínimo impacto visual en el entorno paisajístico.

La relevancia de la actuación y su inevitable afección paisajística obligaron a la búsqueda de un diseño insertado e integrado adecuadamente en el entorno, resultando incluso enriquecedores del mismo. La belleza, las cualidades ambientales y la variedad de matices de la ría orientaron la solución hacia la búsqueda de un diseño en celosía muy transparente, con una transición de cantos serenos, con un cambio de curvatura y forma de vientre de pez cercano a las pilas del cauce, que permitiera al tablero del puente pasar «flotando» sobre la ría (figs. 1 y 2 ), rompiendo con las variaciones de canto clásicas picudas que remarcarían con demasiada rotundidad su apoyo puntual en las pilas centrales.

Los colores elegidos - el color gris perla claro del hormigón y el acero de la celosía pintado en tono verde claro- permiten garantizar la discreta y elegante inserción del viaducto en el paisaje circundante, donde predominan los colores verdes de los bosques y azules grisáceos del río, tamizados y matizados por los reflejos brillantes de los días luminosos o las brumas y nieblas frecuentes en esta tierra.

\section{Concepto estructural y descripción del viaducto}

Los condicionantes descritos en el apartado anterior orientaron la solución hacia una celosía mixta continua de canto variable, con doble acción mixta en las zonas de flexión negativa, tres grandes vanos principales de $225+240+225 \mathrm{~m}$ de luz y vanos tipo de acceso de $120 \mathrm{~m}$ de luz, lo que supone un vano principal con una luz un $20 \%$ superior al anterior récord del mundo de su tipología, el puente de Nantenbach sobre el río Main [2], en Alemania, con un vano central de $208 \mathrm{~m}$ de luz.

$\mathrm{El}$ viaducto tiene una longitud total de $1.620 \mathrm{~m}$ con una distribución de luces de $50+80+3 \times 120+225+240+$

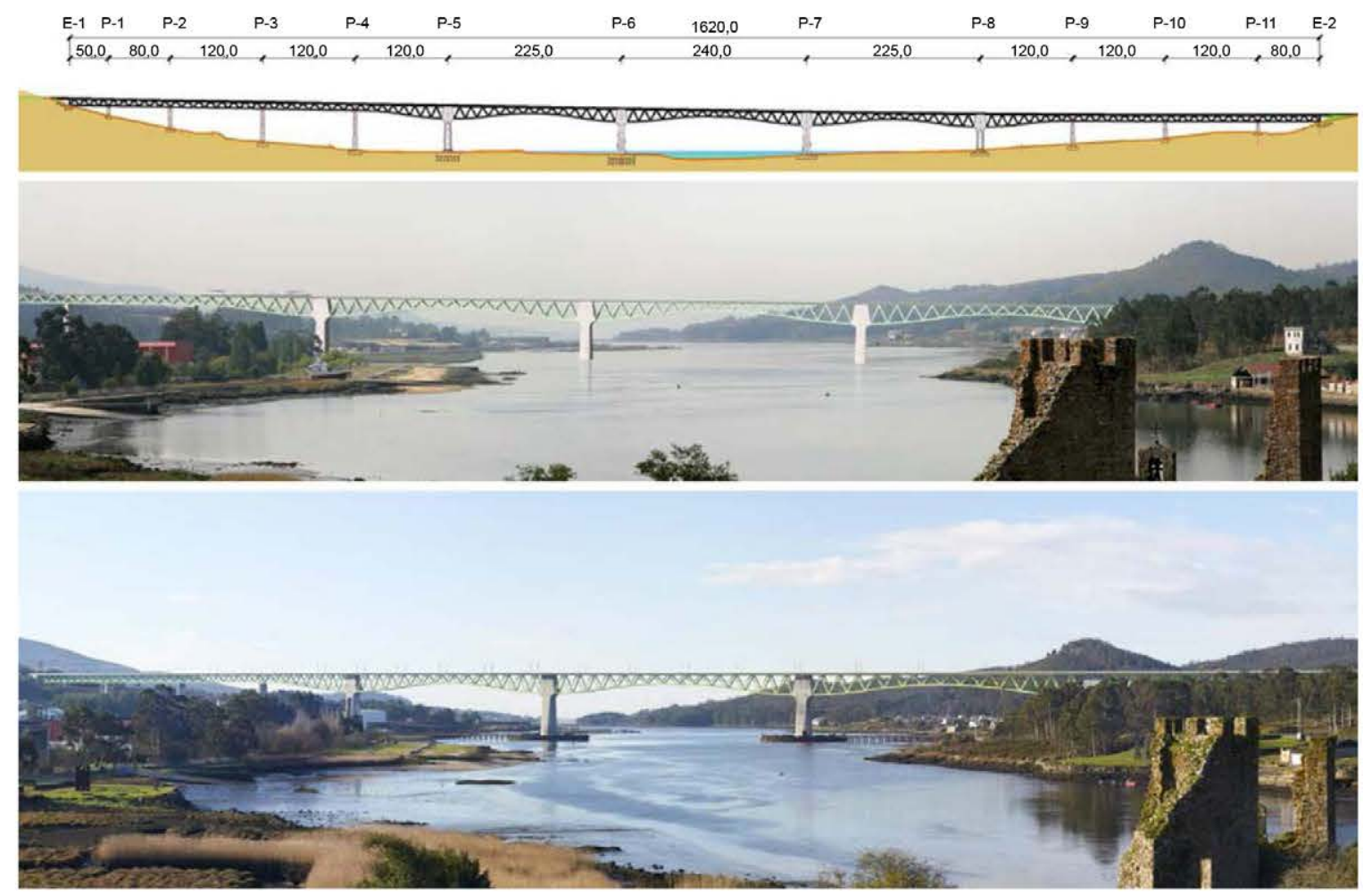

Figura 1. Alzado, fotomontaje y vista del viaducto sobre el río Ulla concluido. 


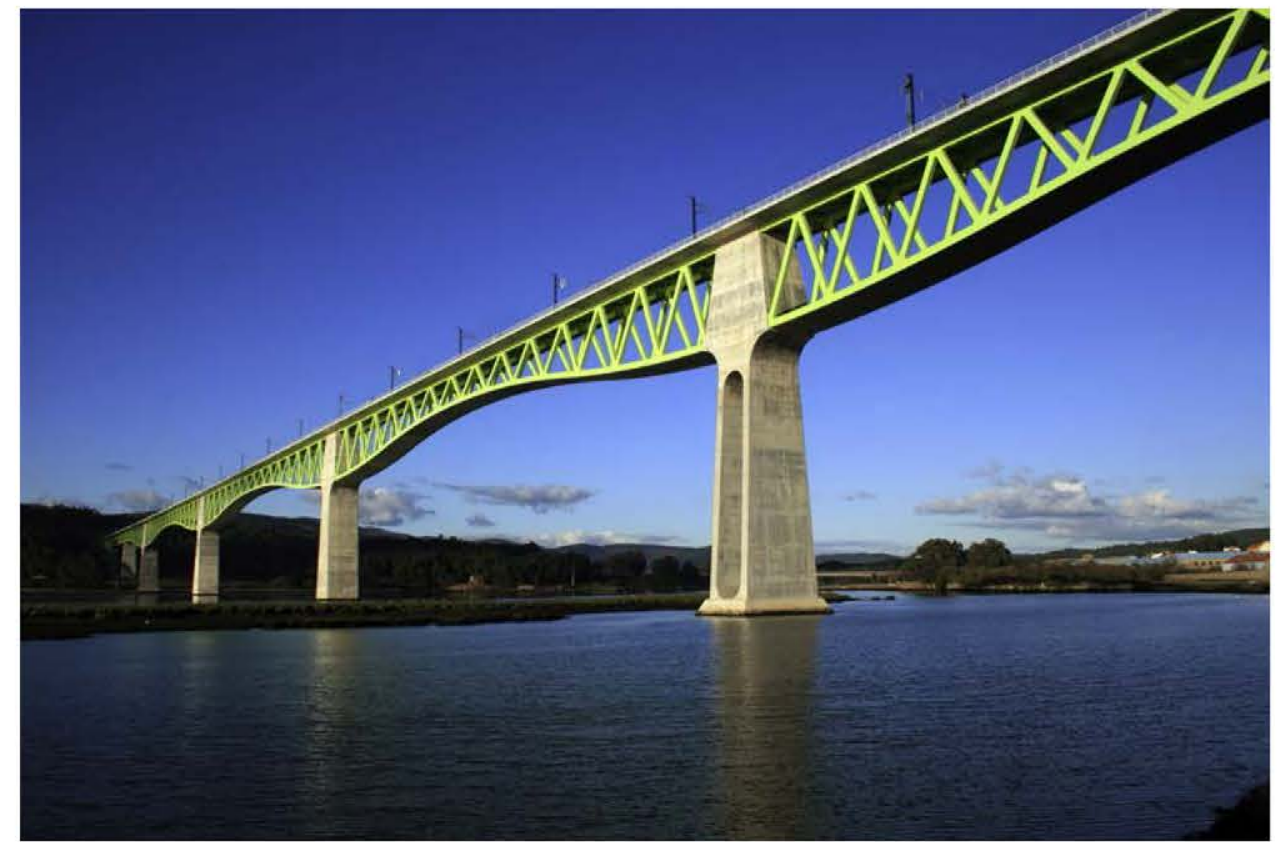

Figura 2. Imagen de los vanos de canto variable centrales del viaducto. Pila 5 en primer término.

$225+3 \times 120+80 \mathrm{~m}$ (fig. 1), y se disponen dos juntas con aparato de dilatación de vía en ambos estribos.

Los cinco vanos principales de canto variable (fig. 2) tienen $17,90 \mathrm{~m}$ de canto total sobre apoyos (17,5 $\mathrm{m}$ de canto de metal) y $9,15 \mathrm{~m}$ ( $8,75 \mathrm{~m}$ de metal) en centro de vano. Los vanos de los tramos de acceso, con luces tipo de $120 \mathrm{~m}$, mantienen la sección tipo en celosía mixta pero con canto constante de $9,15 \mathrm{~m}$ de canto total.

Las cuatro pilas centrales ( $\mathrm{P}-5$ a $\mathrm{P}-8)$, de cuidado diseño en forma de copa, se vinculan rígidamente a la celosía mixta del tablero, configurando un pórtico mixto con la suficiente rigidez en los tres vanos centrales, de $225+240+225 \mathrm{~m}$ (fig. 2), para hacer frente a las exigencias de deformación de la alta velocidad bajo la acción de sobrecargas que actúan sobre vanos alternos.

La rigidez de las cuatro pilas principales se ha optimizado de forma que, al mismo tiempo que confieren la necesaria coacción frente a los giros del tablero, controlan el nivel de flexión que, a través del efecto pórtico, se transmite a la cimentación de las pilas, evitando así su sobredimensionamiento [3].

Por ello, las pilas principales laterales (P-5 y P-8, fig. 2), situadas en el extremo exterior de los pórticos de $225 \mathrm{~m}$ de luz, se han proyectado con dos tabiques exentos empotrados en la cimentación y cabeza de pila. Se han controlado así los niveles de empotramiento a flexión derivados de la fuerte descompensación de luces, de 225 y $120 \mathrm{~m}$, de los vanos de tablero adyacentes, así como las flexiones derivadas de los desplazamientos impuestos en cabeza de carácter térmico y reológico, sensiblemente superiores a los de las dos pilas centrales (P-6 y P-7) a causa del considerable incremento de su distancia al punto neutro de desplazamientos, del orden de $350 \mathrm{~m}$.

El resto de las pilas de los vanos de acceso son convencionales, con sección rectangular con sección hueca y dimensiones variables en longitudinal y transversal. Los apoyos del tablero sobre dichas pilas son aparatos de apoyo esféricos libres longitudinalmente y con desplazamiento transversal impedido en uno de ellos.

La celosía tipo Warren se modula en segmentos de $15 \mathrm{~m}$ con los nudos del cordón superior separados en transversal $6 \mathrm{~m}$ y las diagonales inclinadas en la zona de canto constante unos $45^{\circ}$ respecto de la horizontal. Los cordones superior e inferior son paralelogramos formados por chapas de acero, con $0,80 \mathrm{~m}$ de ancho y cantos de 1,00 y $1,20 \mathrm{~m}$, respectivamente. Las diagonales tienen una sección similar a la del cordón superior.

Los cordones superiores presentan, soldada a su ala superior, una cabeza superior cerrada adicional, embebida en la losa de hormigón, a la que se sueldan los conectadores lateralmente, lo que permite aproximar el eje de transferencia de la conexión al baricentro de la losa superior, reduciendo así las flexiones parásitas por excentricidad en la citada conexión (fig. 3).

El acero de la celosía es de calidad S355-J2+N y S355-K2+N (para chapas superiores a $60 \mathrm{~mm}$ ) para los vanos de acceso y de calidad termomecánica S-460-M y S-460-ML (para chapas superiores a $65 \mathrm{~mm}$ ) en los tres vanos centrales de canto variable y mayor luz.

La losa superior tiene un canto variable entre 0,46 y $0,25 \mathrm{~m}$, y el hormigón in situ, HA-35, se ejecuta sobre prelosas prefabricadas colaborantes con el ancho completo de la sección transversal.

El hormigón de fondo, HA-50, se ubica entre los cordones metálicos inferiores de la celosía, conectándose a los mismos para materializar la doble acción mixta en las zonas sometidas a flexión negativa. En las zonas de centro de vano se da continuidad al hormigón inferior con un cierre simplemente formal no estructural, para garantizar y facilitar las tareas de inspección y mantenimiento (fig. 4). 

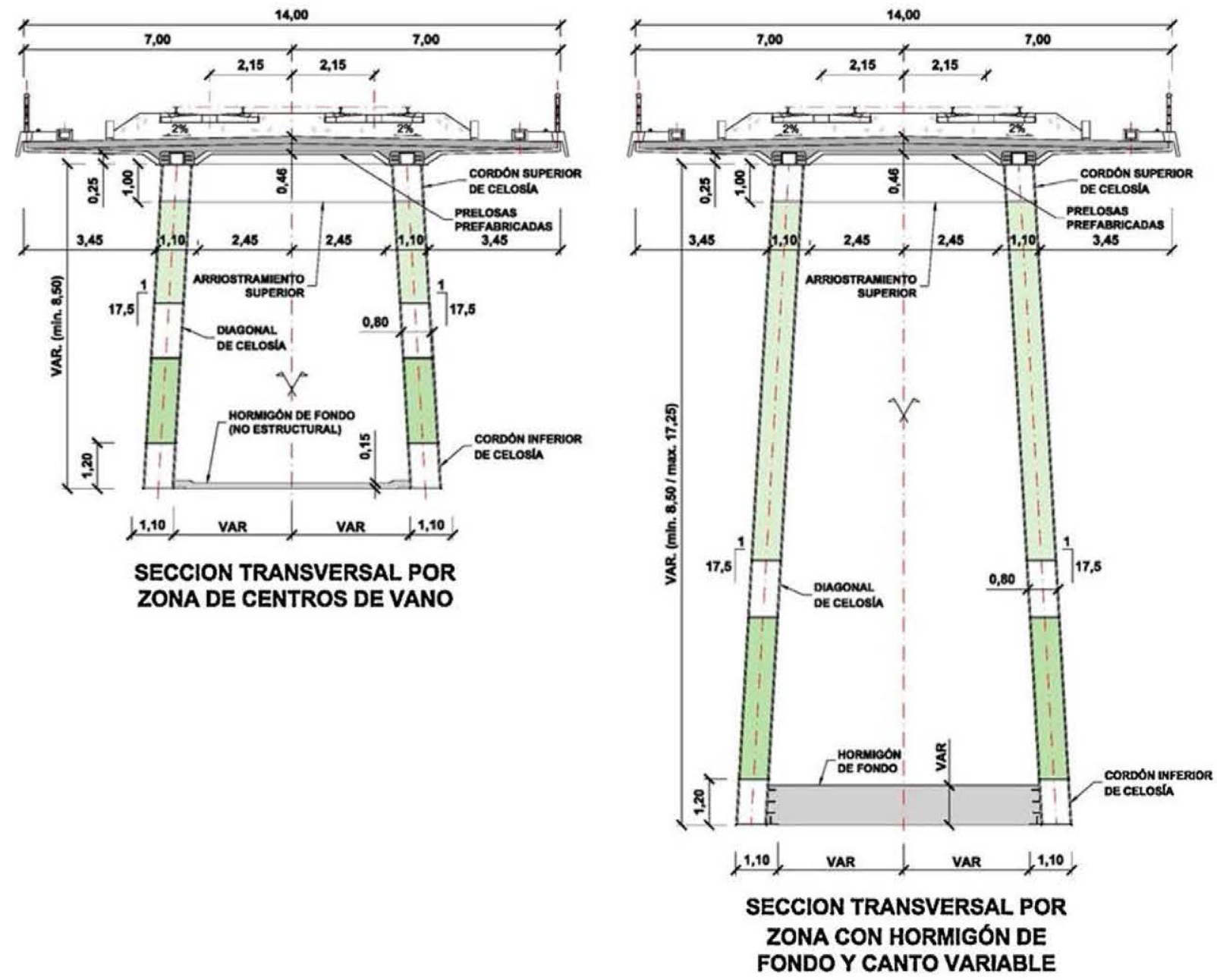

Figura 3. Secciones transversales tipo.

3. Descripción del proceso constructivo de las cimentaciones

La geología de la traza muestra una sucesión de niveles en la que primero se encuentran unos suelos de recubrimiento de escasa compacidad, de origen coluvial en las laderas y aluvial en la ría, bajo los que se encuentra el sustrato rocoso granítico, más o menos alterado en su capa más superficial.

Los estribos y las pilas P1 a P4 y P8 a P11, con accesos sencillos por vía terrestre, se cimentan mediante zapatas apoyadas
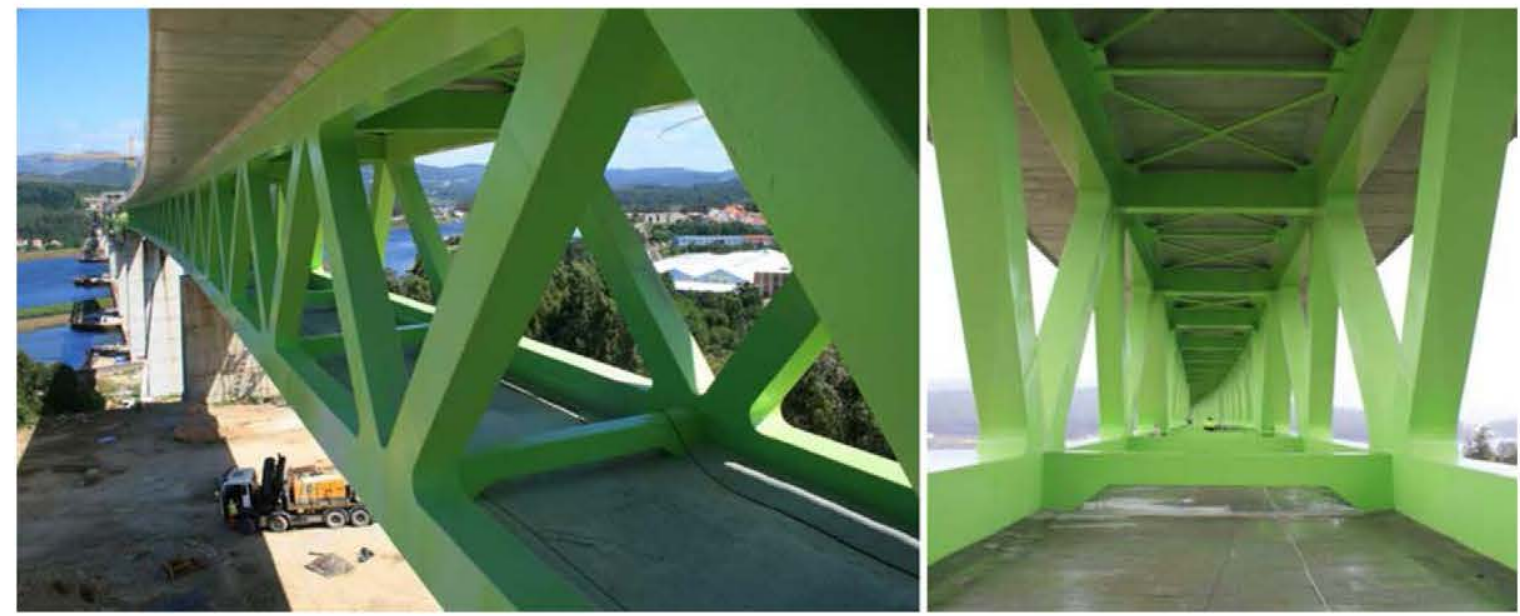

Figura 4. Vista lateral y del interior de la sección transversal desde el hormigón inferior. 


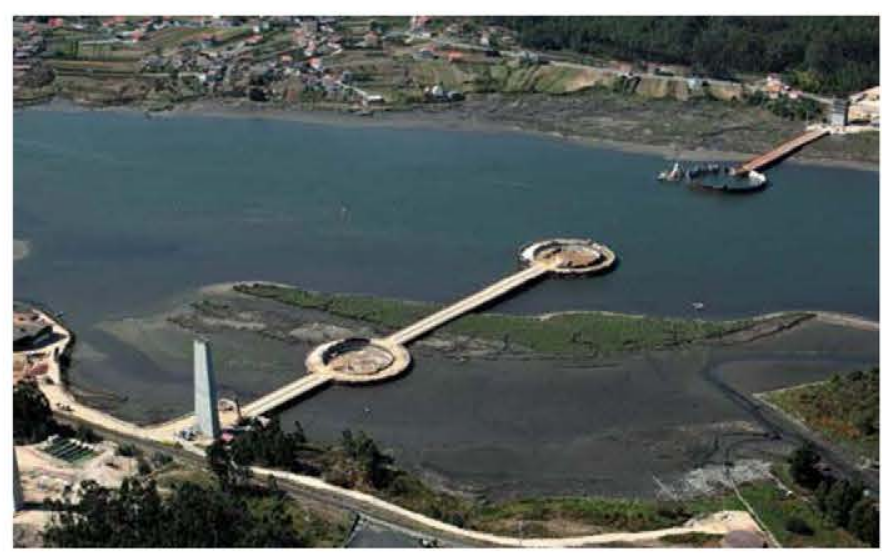

Figura 5. Vista del pantalán y los recintos de las cimentaciones de las pilas P-5, P-6 y P-7 en el cauce.

sobre el estrato granítico con una tensión media admisible de $1,00 \mathrm{MPa}$. De las tres pilas situadas en la ría (pilas 5, 6 y 7), las dos primeras (pilas 5 y 6 ) se cimentan con encepados de pilotes de $1,5 \mathrm{~m}$ de diámetro empotrados en el granito sano, mientras que la tercera (pila 7) se apoya en una cimentación superficial sobre el sustrato rocoso.

\subsection{Pantalán provisional de acceso a las cimentaciones en el cauce}

Para el acceso a las cimentaciones de las tres pilas del cauce (fig. 5), así como para permitir el transporte de las dovelas metálicas del tablero y su posterior izado, se ejecutó un pantalán provisional que ha permitido realizar la obra casi íntegramente con medios terrestres, mejorando los rendimientos ostensiblemente y respetando las zonas de las márgenes protegidas medioambientalmente.

El tablero del pantalán está formado por una sucesión de vanos isostáticos metálicos de $6 \mathrm{~m}$ de luz y $14 \mathrm{~m}$ de anchura (fig. 6) que permiten separar el tráfico en dos carriles de 4,5 m de anchura, una mediana de $1 \mathrm{~m}$ y dos bandas laterales de $2 \mathrm{~m}$ de anchura divididas en dos mitades, para la colocación de tuberías de suministro a los recintos y para la circulación de peatones. El tablero metálico se coloca a su vez sobre unos dinteles que se apoyan dos pilotes metálicos de sección tubular de $800 \mathrm{~mm}$ de diámetro y $12 \mathrm{~mm}$ de espesor, que se hincan mediante martillo de impacto hasta llegar a rechazo. La referencia [4] describe con detalle la sección tipo y el proceso constructivo empleado para la ejecución del pantalán.

\subsection{Recintos provisionales de tablestacas en las cimentaciones en el cauce}

En las tres pilas del cauce se ha construido un recinto provisional (fig. 5) para poder ejecutar las cimentaciones en seco por debajo del nivel del agua. A su vez los recintos se han empleado como plataforma de trabajo para ejecutar las pilas y permitir el transporte e izado de dovelas del tablero metálico.

Los tres recintos se han ejecutado mediante un doble ani1lo, concéntrico, de tablestacas metálicas separadas $10 \mathrm{~m}$ entre sí de $62 \mathrm{~m}$ de diámetro en P-5, $68 \mathrm{~m}$ en P-6 y $65 \mathrm{~m}$ en P-7, trabajando a flexión con arriostramientos circulares a distintos niveles [4]. Los exteriores son metálicos, trabajando a tracción, y los interiores son de hormigón armado, trabajando a compresión.

En las pilas 6 y 7 las tablestacas interiores se han hincado hasta la roca. La profundidad de excavación es de $8 \mathrm{~m}$ en P-5, de 10,5 m en P-6 y de $13 \mathrm{~m}$ en P-7 (fig. 7), y en pleamar máxima la cota del nivel de la ría se sitúa a la $+2,54 \mathrm{~m}$, muy próxima a la cota de la plataforma de los recintos $(+3,0 \mathrm{~m})$. Esta importante profundidad libre de excavación, en el entorno de $\operatorname{los} 15 \mathrm{~m}$, unido a la presencia exterior de agua, ha obligado a realizar contenciones muy importantes de los recintos.

El recinto exterior se ha arriostrado mediante unos zunchos metálicos perimetrales compuestos por vigas armadas de acero S-355. En las pilas 5 y 6 se ha colocado un zuncho y en la pila 7 han sido necesarios dos (fig. 7).

Los arriostramientos comprimidos de las tablestacas interiores se han materializado mediante anillos de HA-40 y sección rectangular de $1,0 \times 1,0 \mathrm{~m}$. En las pilas 5 y 6 han sido necesarios tres niveles de arriostramiento (fig. 8), y en la P-7 han hecho falta cuatro.

Adicionalmente, en todos los recintos se ha ejecutado una doble corona de columnas de suelo tratado mediante jet-grouting alrededor del recinto interior de tablestacas que se ha empleado para sellar el contacto entre la roca y las tablestacas, evitando
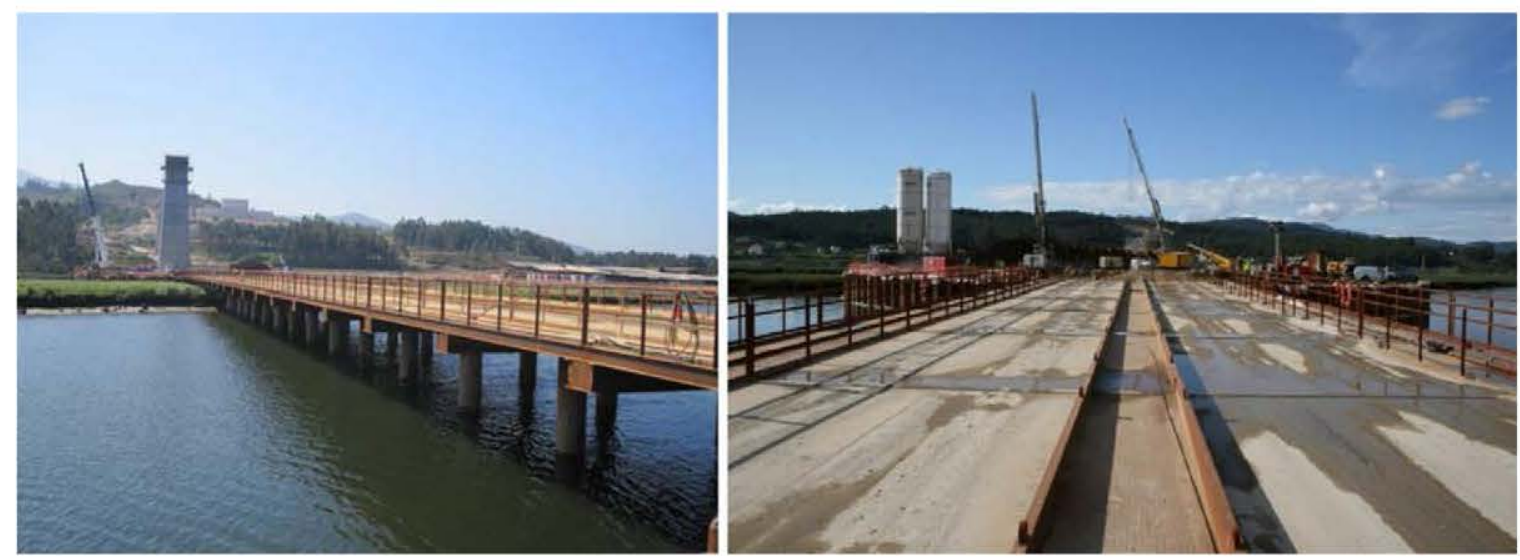

Figura 6. Vistas del pantalán de acceso a las cimentaciones de las pilas P-5, P-6 y P-7. 


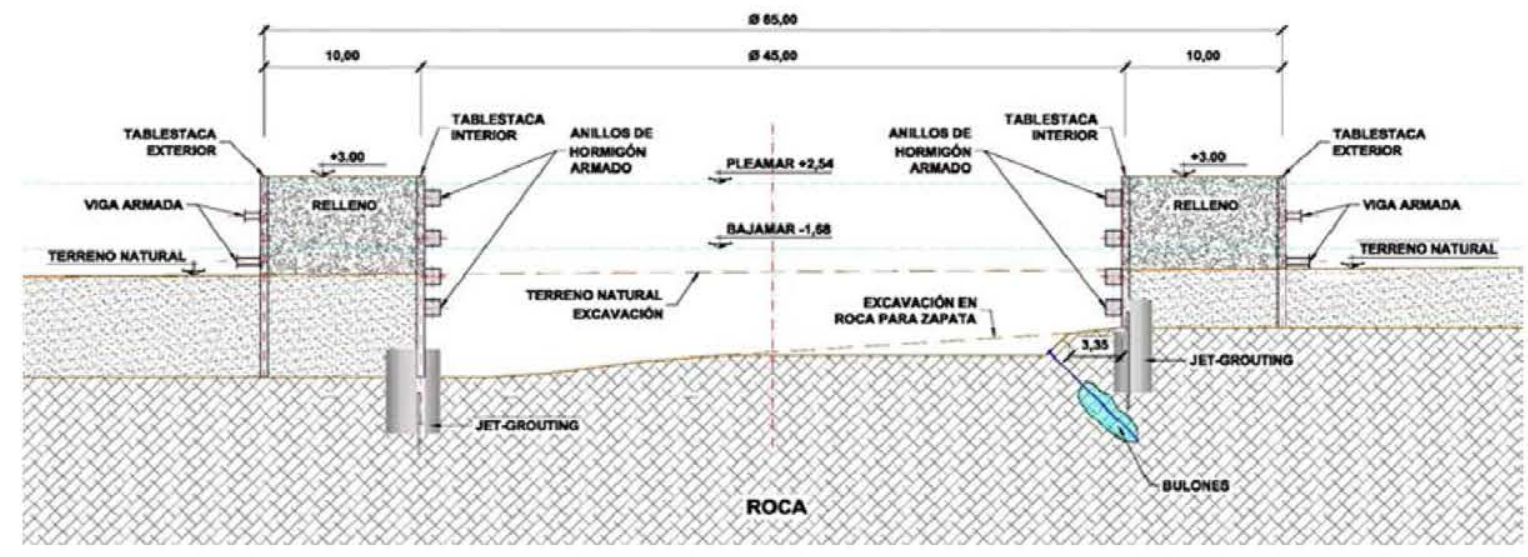

Figura 7. Esquema del recinto de tablestacas de la pila 7.

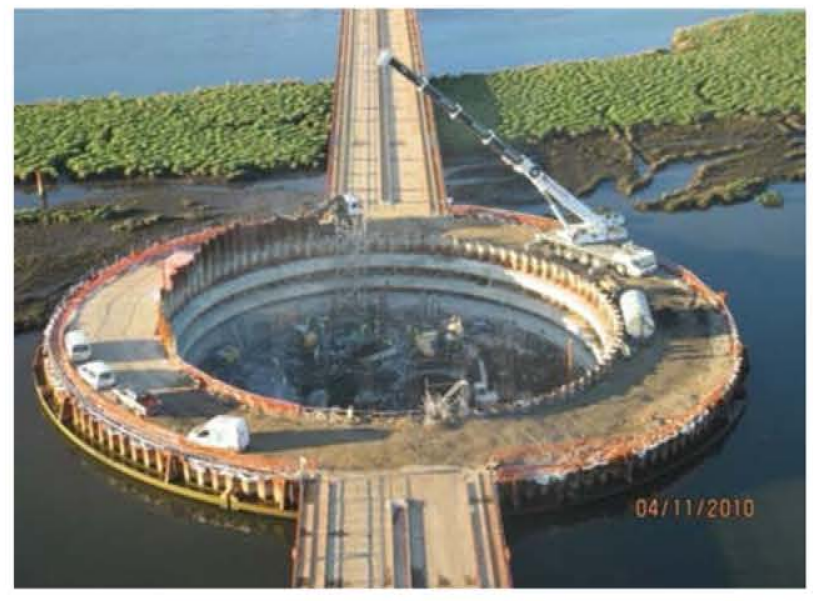

Figura 8. Excavación del interior del recinto de P-5.

filtraciones. Además, en la pila 6 su misión ha sido también estructural, ya que se ha aprovechado la capacidad resistente de su forma circular para sustentar lateralmente la punta de las tablestacas.

En la pila 7 se necesitaba más resistencia para contener las puntas de las tablestacas interiores, ya que se descalzaban durante la excavación, por lo que se ha ejecutado un micropilote armado en el pie de cada una de las tablestacas para resistir este esfuerzo cortante (fig. 7).

\subsection{Ejecución de los recintos y de las cimentaciones en el cauce}

La ejecución de los tres recintos de tablestacas se ha realizado hincando por vibración las tablestacas exteriores desde pontona y posteriormente se han arriostrado con los zunchos metálicos exteriores.

El relleno del recinto interior se hizo vertiendo el material desde camiones, con acceso por el pantalán, habiéndose dispuesto previamente un forro con un geotextil por el interior para reducir al máximo el escape de finos hacia la ría.

Posteriormente se hincaron las tablestacas de los recintos interiores desde la superficie del relleno, una vez concluido se realizó el tratamiento de superjet-grouting en los pies de las tablestacas y finalmente se ejecutaron los micropilotes (en el caso de la P-7) desde la plataforma.

La cimentación de la pila 5 se ha ejecutado mediante un encepado de 42 pilotes de $1,5 \mathrm{~m}$ de diámetro y la de la pila 6 con 56 pilotes, empotrados $1,5 \mathrm{~m}$ en el sustrato granítico de grado de meteorización I. En ambos casos los pilotes se han ejecutado desde la superficie del relleno del recinto a cota $+3,0 \mathrm{~m}$. Se ha perforado al amparo de una camisa recuperable hasta llegar a la roca, evitando la utilización de lodos para evitar posibles fugas en la ría. La camisa se ha hincado bien hasta el contacto con el sustrato rocoso, más o menos alterado, o hasta el jabre de compacidad muy densa en los casos en que este ha aparecido. Una vez ejecutados todos los pilotes, se ha procedido a la excavación de los recintos (fig. 8), realizando los arriostramientos interiores correspondientes en cada escalón, y al descabezado de los pilotes (fig. 9) para proceder al ferrallado (fig. 10) y hormigonado de los encepados.

El encepado de la pila 5 tiene unas dimensiones de $30 \times 24 \times 4,5 \mathrm{~m}$ (fig. 10 ) y el de pila 6 , de $34,5 \times 30,5 \times 5 \mathrm{~m}$. La cota inferior del encepado es la $-5,0 \mathrm{~m}$ para la pila P-5 y de $-7,5 \mathrm{~m}$ para la pila 6 .

Dadas las dimensiones de los encepados, el mayor de $5.260 \mathrm{~m}^{3}$, el hormigonado de los mismos ha sido una tarea de notable relevancia. El hormigonado de las cimentaciones de las tres pilas del cauce ha sido continuo, en algún caso durante más de $50 \mathrm{~h}$, y se han utilizado varios tipos de hormigones dependiendo de la zona del encepado. En los $0,7 \mathrm{~m}$ inferiores se ha empleado HA-35 autocompactable para mejorar su puesta en obra en la zona de las densas parrillas inferiores de armado; en la zona intermedia del encepado se ha empleado HA-35 convencional vibrado, y en la capa superior, de un metro de profundidad, se ha empelado HA-35 con fibras para evitar la fisuración superficial. Además, en la zona central de entronque con el cáliz de la pila el hormigón empleado tiene la misma calidad que la pila, HA-70. Los pilotes, de hormigón HA-35, se han diseñado para trabajar a $8 \mathrm{MPa}$ en servicio.

La pila 7 se cimenta mediante una zapata de $27 \times 24 \times 5,25 \mathrm{~m}$. La cota de la cara inferior de la cimentación es la $-8,6 \mathrm{~m}$. Se apoya sobre el sustrato granítico de grado de alteración menor o igual a III mediante un relleno de hormigón pobre HM-15 trabajando a una tensión cobaricéntrica 


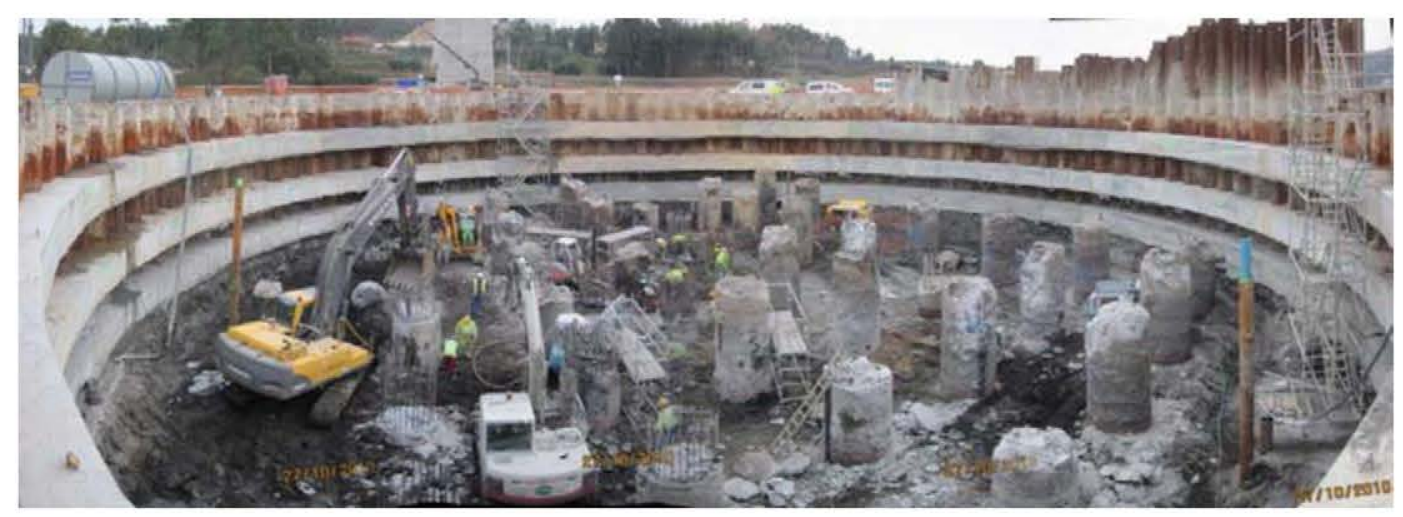

Figura 9. Descabezado de pilotes de la cimentación de P-5.

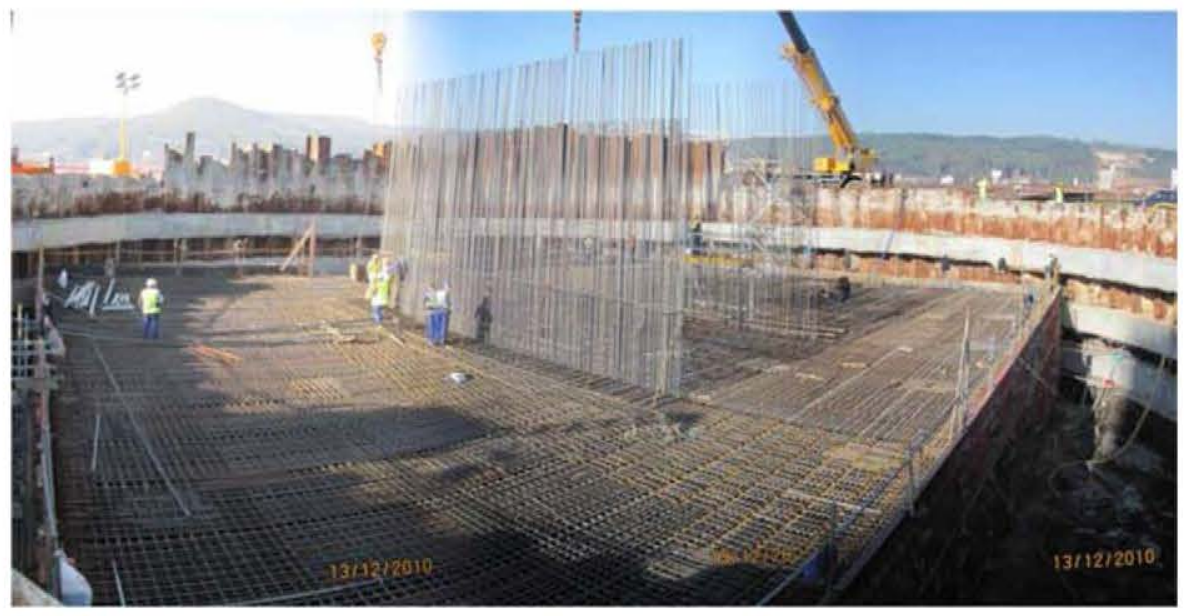

Figura 10. Vista de la ferralla del encepado de P-5 $(30 \times 24,5 \times 4,5 \mathrm{~m})$.

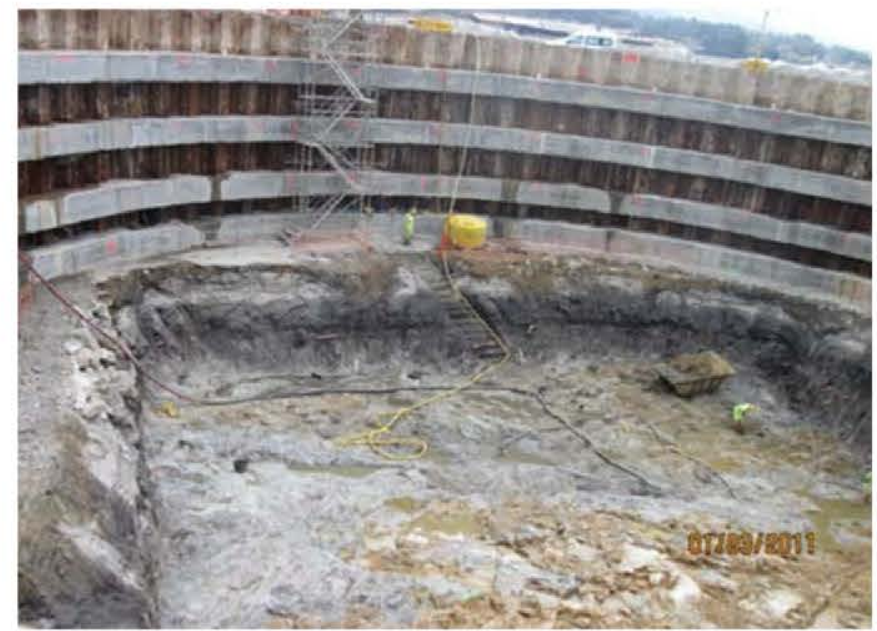

Figura 11. Vista del fondo rocoso de P-7 previo hormigón de limpieza $(27 \times 24 \times 5,25 \mathrm{~m})$.

máxima de $0,76 \mathrm{MPa}$, con una tensión de punta máxima de 1,00 MPa (fig. 11).

Una vez ejecutadas las cimentaciones del cauce, se rellenan los recintos interiormente demoliendo los zunchos interiores y siguiendo, según se va rellenando, el proceso inverso al de su ejecución. Una vez finalizado el relleno, se extraen las tablestacas del recinto interior dejando las islas listas para el trasporte e izado de dovelas del tablero.

La cota superior de los encepados de P-5 y P-6 y de la zapata de P-7 queda por debajo del fondo natural del cauce del orden de un metro en su parte más superficial. Una vez concluido todo el tablero, se procede a la retirada del relleno interior, de los recintos exteriores y del pantalán, restituyendo el aspecto original de la ría.

\section{Fabricación de la estructura metálica del tablero}

La singularidad y complejidad de la estructura metálica del viaducto, así como el gran volumen de acero estructural, con cerca de $20.000 \mathrm{t}$, indujo al Contratista a proponer a la Dirección de Obra el reparto de la fabricación entre dos grupos de talleres. Inicialmente se pensó en fabricar medio viaducto en los talleres de Ascamón y Joama, en Asturias, y la otra mitad en los talleres portugueses de Martifer. La complejidad de la obra y los plazos de ejecución obligaron posteriormente a la inclusión de dos talleres adicionales en Galicia: Emesa y Dizmar (fig. 12).

Con objeto de poder manipular, fabricar y transportar los elementos metálicos a obra, las celosías metálicas del tablero se concibieron desde la fase de proyecto descompuestas en los elementos simples siguientes (fig. 13): nudos superiores, cordones 


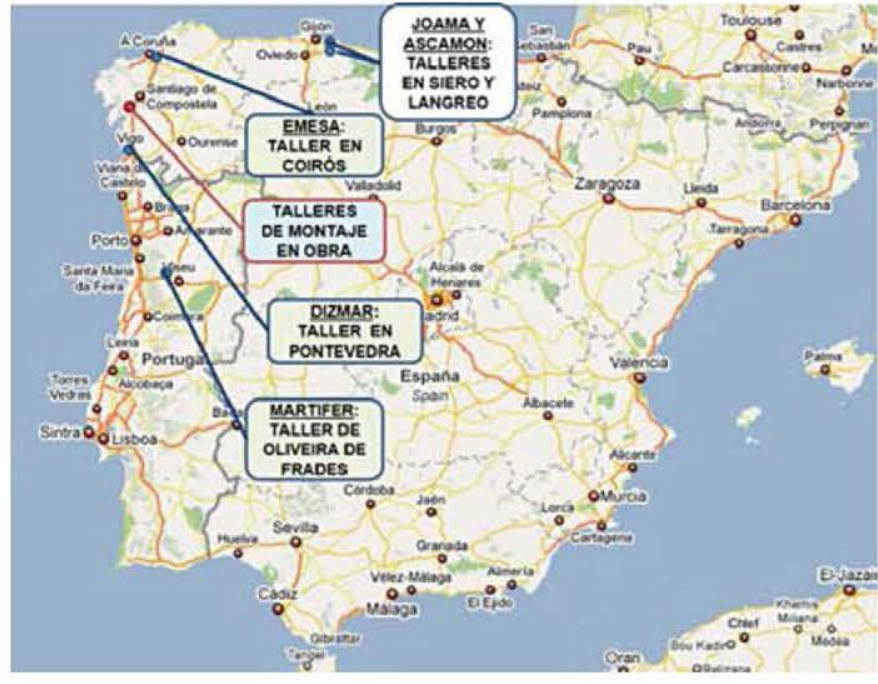

Figura 12. Ubicación de talleres que han participado en la ejecución de la estructura metálica del viaducto.

superiores, diagonales, nudos inferiores, cordones inferiores, montantes horizontales y arriostramientos en cruz.

Una vez ejecutados estos elementos individuales de cada dovela, en función de cada caso y previamente al transporte a obra, se sueldan en subconjuntos mayores: nudo+cordón, o nudo+cordón+nudo. Estos subconjuntos y el resto de elementos simples (diagonales, montantes y arriostramientos) se transportan a obra para posteriormente terminar de ensamblar, en los parques de montaje de obra, las dovelas de la celosía.

Para poder realizar las labores de ensamblaje de las piezas individuales y los subconjuntos armados en taller, se han tenido que ejecutar tres grandes talleres de ensamblaje de dovelas (fig. 14) con capacidad todos ellos para montar dovelas en obra de hasta 17,5 m de canto, además de unas amplias zonas de acopio y montaje cerca del E-1, y en la sombra del viaducto entre las pilas P-9 y el E-2.

\subsection{Planteamiento y organización del control de calidad de la estructura metálica}

El Pliego de Prescripciones Técnicas Particulares (PPTP) del Proyecto establecía las bases generales que han servido para desarrollar en obra el control de ejecución de la estructura metálica [5]. La base de todo el sistema se fundamenta en asignar al Contratista la responsabilidad de acreditar la conformidad de la correcta ejecución de la estructura metálica, a través de su Programa de Control de Calidad, de acuerdo a los requisitos de las normativas de ejecución de estructuras metálicas, para lo cual el PPTP establecía la posibilidad de que el Contratista se encargase directamente de realizar, y acreditar, todo el control establecido en el Pliego o bien asumir como suyos los autocontroles del taller metálico y contrastarlos con al menos un $20 \%$ de ensayos y controles de contraste. El precio de la unidad incluía, por tanto, todos los costes relativos a la realización de estas tareas de control por parte del Contratista.

El marco de juego establecido en el PPTP ha obligado al Contratista a exigir a cada uno de los talleres a cumplir con el $100 \%$ de los requisitos de control establecidos en este, y a contar con una Entidad de Control independiente, con presencia permanente en los talleres y en la obra, que se ha responsabilizado de la acreditación, en su nombre, frente a la Dirección de Obra de la conformidad de la ejecución de la estructura metálica.

Esta Entidad de Control independiente ha supervisado y validado todo el proceso de ejecución de la estructura metálica en talleres y en obra, desde el encargo y recepción de los materiales, las homologaciones de soldadores y cualificaciones de los procedimientos de soldadura, pasando por la revisión de todos los controles de ejecución y ensayos no destructivos (END) que realizan los talleres.

Además de supervisar y auditar continuamente a los talleres metálicos y su sistema de control de calidad, la Entidad de Control del Contratista (Alfainstant y luego AMT) ha realizado al menos un $20 \%$ de controles de contraste. Estos controles de contraste no se limitan solo a repetir ensayos no destructivos (líquidos penetrantes, partículas magnéticas, ultrasonidos, o radiografías), sino que se ha incidido, de forma muy intensa, en la fase de control de ejecución: corte de chapas, preparaciones de bordes (biseles), controles dimensionales, controles visuales previos a la soldadura, controles geométricos y de los procesos de montaje en blanco, etc.

Adicionalmente al sistema de control del Contratista, la Dirección de Obra ha contado, además, con una Asistencia Técnica (TYPSA), en la que está incorporado el proyectista como asesor especializado en estructuras metálicas (IDEAM),

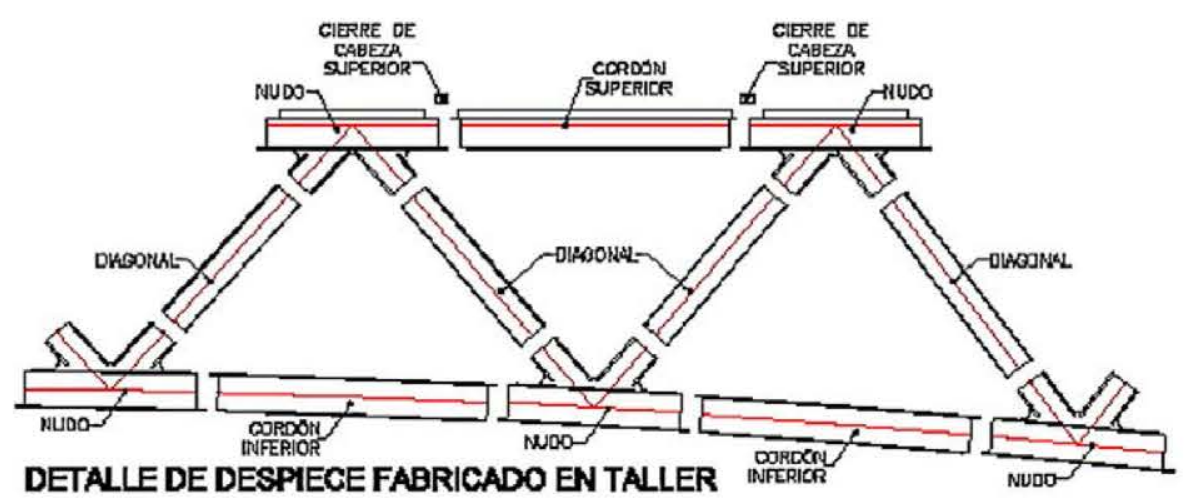

Figura 13. Despiece de elementos simples en la fabricación en taller. 

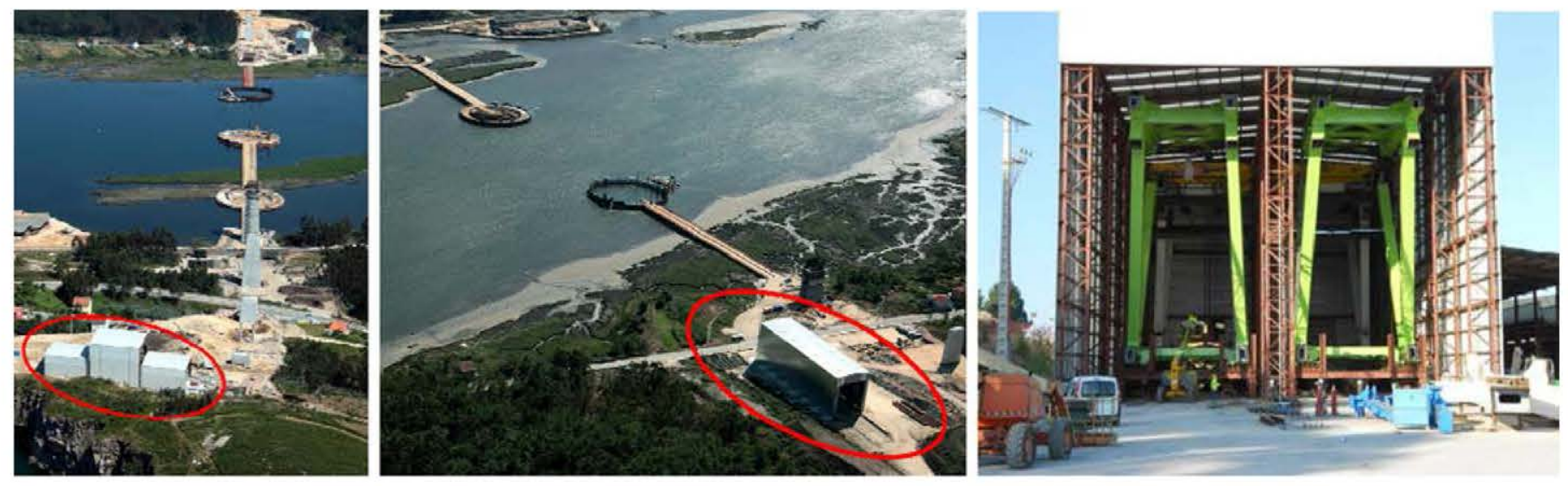

Figura 14. Talleres de obra para ensamblaje de dovelas.

así como con otra Entidad de Control de Calidad independiente (Applus), que se ha encargado de auditar todo el sistema de control de ejecución del Contratista, además de su contraste adicional (del orden del 20\%), con el apoyo de un Laboratorio de Control que además ha realizado también END aleatorios de contraste.

Todo el sistema de control del Contratista ha quedado perfectamente recogido en tres Programas de Puntos de Inspección (PPI), de ejecución en taller metálico, de montaje en los talleres de obra y de montaje in situ en obra, en los cuales se establecen los porcentajes de control que realiza el taller metálico mediante su autocontrol, junto con los controles de contraste que realiza la Entidad de Control del Contratista.

El Plan de Aseguramiento de Calidad (PAC) del Contratista establece los sistemas y mecanismos de control, así como el organigrama y las funciones y responsabilidades de todos los agentes implicados en la fabricación y el control de calidad, y fija también los mecanismos para abrir incidencias de ejecución y no conformidades.

Una incidencia de ejecución es un fallo o no conformidad detectada por el taller metálico durante alguna de las fases del proceso de fabricación de la estructura metálica. Una incidencia se comunica siempre, independientemente de su importancia, a la Entidad de Control del Contratista y a la Entidad de Control de la Dirección de Obra. Si la importancia es menor, el taller metálico informa sobre la incidencia, la documenta y la resuelve, $\sin$ necesidad de tener que parar la producción. Si, en cambio, la incidencia presenta mayor transcendencia, se comunica a las dos Entidades de Control y se consensua la solución mediante la propuesta previa de reparación por parte del taller metálico y la Entidad de Control del Contratista.

Un fallo o defecto de ejecución detectado por la Entidad de Control del Contratista que el control de calidad del taller ha pasado por alto, implica una no conformidad.

Una no conformidad se puede abrir, también, si el taller metálico está detectando incidencias de ejecución de manera repetida en un mismo proceso, como por ejemplo un fallo repetitivo en el control dimensional de las piezas, o en el control de ultrasonidos de una soldadura tipo, ya que ello implica que algo está fallando de manera sistemática y requiere una actuación como el replanteamiento del proceso de ejecución o del proceso de control, con mayor calado que la simple reparación puntual de un fallo o defecto individual.

Ante una no conformidad abierta por el Contratista, se debe parar, documentar la no conformidad, analizar la causa y proponer no solo una solución puntual, sino una medida correctora que evite su repetición sistemática. Solo tras la validación de la propuesta por parte de la Entidad de Control de la Dirección de Obra se permite continuar con la reparación y la producción.

En cada uno de los controles establecidos en los PPI se establecen las normativas específicas de control, así como los criterios de aceptación de los controles. Este punto es fundamental coordinarlo antes del inicio de los trabajos, para que todos los estamentos implicados en el control de ejecución tengan claros los criterios para controlar y cuáles son los baremos que sirven para validar cada control específico. En el viaducto del río Ulla se ha decidido tender a un control basado en normas europeas (normas EN) y sus traducciones españolas (UNE-EN). Solo en los casos de no existir una norma EN se ha recurrido a alguna normativa específica de control diferente (principalmente americanas: AWS, etc.).

Finalizado el trabajo de soldadura, solo se autoriza la salida de cada pieza simple o subconjunto de los talleres metálicos, o de cada dovela metálica de las naves de montaje en obra, respectivamente, tras haberse documentado y acreditado por completo la conformidad de todos los controles de ejecución y todos los END establecidos en el PPI correspondiente.

\subsection{Desarrollo de los planos de taller de la estructura metálica}

La complejidad de la obra, la gran dispersión de talleres y los múltiples agentes implicados en el control han conducido a la necesidad de crear, por parte del Contratista, la figura del Coordinador de Ejecución de la Estructura Metálica, quien ha sido el principal interlocutor frente a la Dirección de Obra y su Asistencia Técnica en los aspectos relacionados con la estructura metálica. Entre otras, una de sus principales misiones ha sido la de coordinar cualquier tipo de propuesta técnica de adaptación, modificación o ajuste de algún detalle entre todos los talleres, unificando las propuestas. 
Otro aspecto fundamental en el que se basa todo el control de ejecución es el sistema de aprobación y validación de los planos de taller de la estructura metálica.

Los talleres metálicos desarrollan los planos del proyecto en los planos de taller y las hojas de despiece. El responsable de acreditar la conformidad de los planos de taller y las hojas de despiece es el Contratista, quien a su vez ha contado con un consultor externo especializado que se ha encargado de realizar dicha labor. Una vez que los planos de taller se han aprobado, se remiten a la Asistencia Técnica de la Dirección de Obra, que supervisa los planos de taller y da su visto bueno o bien realiza las consideraciones que se estimen oportunas para su corrección.

La complejidad de la estructura metálica ha requerido un trabajo previo muy importante de estudio y desarrollo de una serie de detalles muy repetitivos, en común entre todas las partes implicadas, para el desarrollo de los planos de taller (talleres metálicos, Contratista y su Apoyo Técnico, junto con el Proyectista que asiste técnicamente a la Dirección de Obra), de manera que los planos de taller resuelven todos los encuentros, soldaduras, transiciones y detalles específicos, evitando así problemas futuros durante la ejecución. La referencia [6] describe con detalle el nivel de definición de los planos de taller de la estructura metálica del viaducto.

En ningún caso se han aceptado detalles tipo, adimensionales o tabulados, que no representen en verdadera magnitud los espesores, geometrías, ángulos de incidencia, separaciones libres, talones y biseles de las chapas en cada uno de los encuentros de soldadura específicos, evitando así un gran número de posibles problemas de ejecución.

Los 131 planos del proyecto, que definen con detalle la estructura metálica, se desarrollan en más de 6.000 planos de taller y cerca de 20.000 hojas de despiece, definiendo con toda exactitud cada una de las chapas, encuentros y soldaduras del puente. Este importante desarrollo de ingeniería ha resultado imprescindible para poder garantizar la correcta concepción de todos los detalles, que deben cumplir requisitos muy estrictos relativos a la resistencia a fatiga al tratarse de un viaducto mixto de alta velocidad.

\subsection{Desarrollo de planos con las secuencias de montaje}

Adicional al desarrollo de los planos de taller y las hojas de despiece, se han desarrollado planos de montaje para cada elemento tipo (nudos superiores, cordones superiores, diagonales, nudos inferiores, cordones inferiores, montantes superiores e inferiores y arriostramientos en cruz), definiendo en ellos:

- Las bancadas de montaje.

- El orden y la secuencia de ejecución de las soldaduras, incluso de los punteos para el armado.

- El procedimiento, el método y las posiciones de soldeo, y los pretratamientos térmicos en las soldaduras.

- Los elementos auxiliares necesarios para arriostrar provisionalmente las chapas de cada elemento.

- Los útiles de volteo de piezas, y todos aquellos elementos auxiliares necesarios para la manipulación.

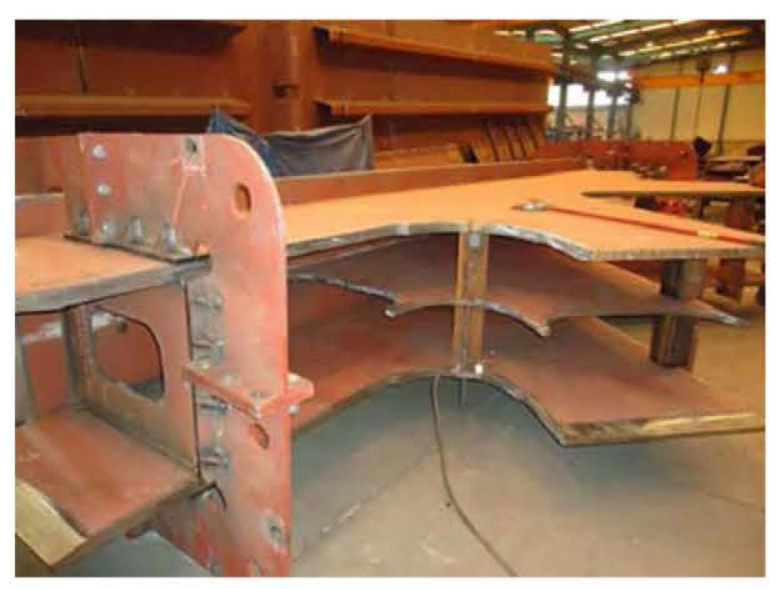

Figura 15. Vista de fabricación de un nudo tumbado.

- Todas las fases intermedias por las que pasa cada elemento simple, subconjunto o dovela durante el montaje.

Cualquier medio auxiliar soldado a un elemento definitivo del puente es susceptible de empeorar la categoría de fatiga de la pieza. Por este motivo se ha concebido la manipulación de cada elemento sin la necesidad de soldar medios auxiliares, gracias al diseño de unos útiles de izado y volteo (fig. 15) que permiten su reutilización en el resto de los diferentes nudos, cordones, diagonales, etc. Esta decisión se ha demostrado además muy acertada y eficaz dada la modularidad de la estructura, con multitud de elementos con geometría exterior idéntica.

De cualquier manera, en los casos en que ha sido necesario disponer orejetas para el montaje en obra de dovelas de mayores dimensiones, siempre se han definido en planos de taller con detalles adecuados a fatiga, y su retirada se ha realizado siguiendo los procedimientos específicos de retirada y reparación con un intenso control posterior.

\subsection{Empalmes de elementos simples y diseño frente a fatiga}

Las uniones entre cordones y nudos se han concebido mediante uniones que evitan 4 soldaduras en la misma sección transversal, decalando las uniones de alas y de almas, materializando una geometría que facilita la colocación de un elemento apoyado sobre el ya soldado. La figura 13 muestra cómo, en general, los nudos se arman con el ala inferior del arranque del cordón volada y con el ala superior retranqueada. Esta geometría se reproduce de forma inversa en los cordones para permitir un más sencillo ajuste y montaje, y evitar cuatro soldaduras en la misma sección (fig. 16).

Esta geometría permite además la realización de despieces con un cupón de ala superior del extremo de los cordones, lo cual facilita la ejecución de tres soldaduras a tope con penetración completa para sanearlas por dentro de la sección transversal, convirtiendo un detalle tipo de fatiga 71 [7] con chapa de respaldo en uno con categoría mínima de 80 con soldadura a tope y saneo de la raíz [7].

Es muy recomendable concebir la estructura metálica, siempre que sea posible, evitando el empleo de soldaduras con acceso 


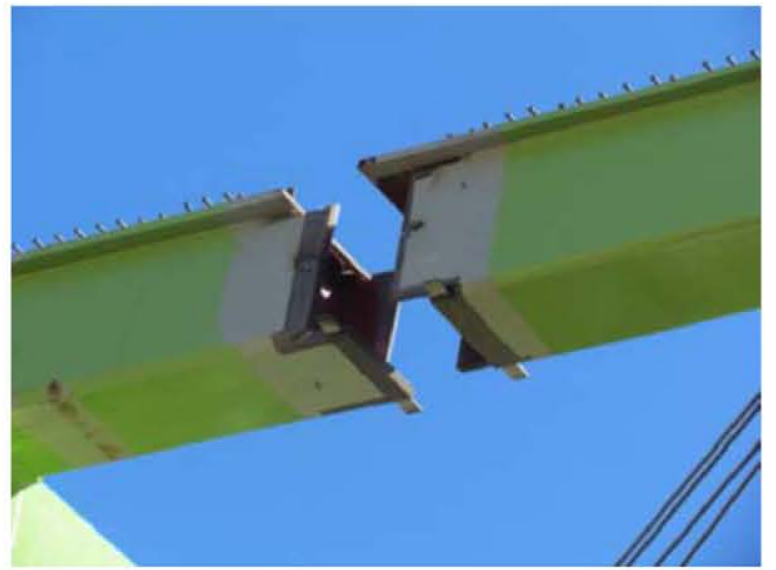

Figura 16. Detalle de empalme de nudo-cordón superior, con soldaduras decaladas.

solo por un lado, lo cual obliga irremediablemente al empleo de chapas de respaldo metálicas. Siempre que sea posible es conveniente ejecutar las soldaduras con acceso por ambos lados con detalles con preparación de bordes en $\mathrm{X}$, o bien en $\mathrm{V}$ mediante el empleo de backing de respaldo provisional cerámico pero con el saneo posterior de la soldadura en la raíz, ya que la ejecución de soldaduras a tope con chapa metálica de respaldo, además de presentar una menor categoría de fatiga, puede generar una serie de problemas que se describen con detalle en [6].

En general, en un puente algo más convencional con sección transversal mediante vigas o cajones mixtos, como son los viaductos mixtos de Alta Velocidad Arroyo las Piedras [8] o de Archidona [9], las soldaduras a tope principales de almas y platabandas casi siempre son accesibles por ambos lados y, por lo tanto, no es necesario tener que recurrir a soldaduras ejecutadas por un solo lado con chapa de respaldo metálica. En cambio, en celosías sí es frecuente tener que recurrir a este detalle, dado que los empalmes entre nudos y cordones o nudos y diagonales suelen tener solo acceso desde el exterior y, por lo tanto, requerir de forma obligada el empleo de chapa de respaldo metálica.

En el viaducto del río Ulla, aunque siempre que ha sido posible se ha intentado minimizar este detalle, quedan muchas uniones, como las del empalme de diagonales con nudos (fig. 13) o las de cierre de cupones de alas en los empalmes de cordones con nudos, que hacen obligado el empleo del backing metálico. En la referencia [6] se describen con detalle las precauciones que es necesario adoptar para la ejecución y el control de ejecución de las soldaduras a tope con acceso por un solo lado con chapa de respaldo, de forma que se pueda garantizar que la categoría de fatiga del detalle realmente ejecutado corresponda con la categoría 71 acorde con UNE-EN 1993-1-9 [7].

\subsection{Detalles de fatiga en encuentros y transiciones}

Con una estructura en celosía con nudos cada $15 \mathrm{~m}$, los detalles de encuentros son muy repetitivos, con ligeros cambios en las dimensiones de las chapas a unir y en las soldaduras, por lo que es muy importante hacer una adecuada concepción con un diseño pensado en detalles con una adecuada categoría de fatiga.

Un claro ejemplo del desarrollo de los principales detalles muy repetitivos son los siguientes, que están completamente definidos desde el origen en los planos de taller para cada nudo:

- La imagen izquierda de la figura 17 muestra el detalle de transición de la soldadura en ángulo entre el ala y el alma de una diagonal o un cordón en su confluencia con el nudo de la celosía donde el ala de la diagonal o del cordón pasa a soldarse con el alma del nudo a penetración parcial, y aparece un doble ojal en planta y alzado, que se resuelve con transiciones y amolados suaves de los extremos de las soldaduras. Este detalle, adecuado frente a fatiga, obligaba al sellado de 12 ojales en cada nudo. Para evitar tal cantidad de chapas de sellado, que pueden convertirse en un punto débil frente a la durabilidad, se decidió hacer una transición suave con pendientes de 1/5 entre la soldadura en ángulo fuera de la parte central de un nudo y la soldadura con penetración parcial en la zona central del nudo, como se puede apreciar en la imagen izquierda de la figura 17.

- El detalle de la transición del alma central en los nudos requiere pasar de 2 soldaduras con penetración parcial y biseles solo en el ala desmembrada, a 2 penetraciones parciales con biseles en el ala desmembrada y en el extremo del alma central, lo cual requiere ejecutar con transiciones suaves con
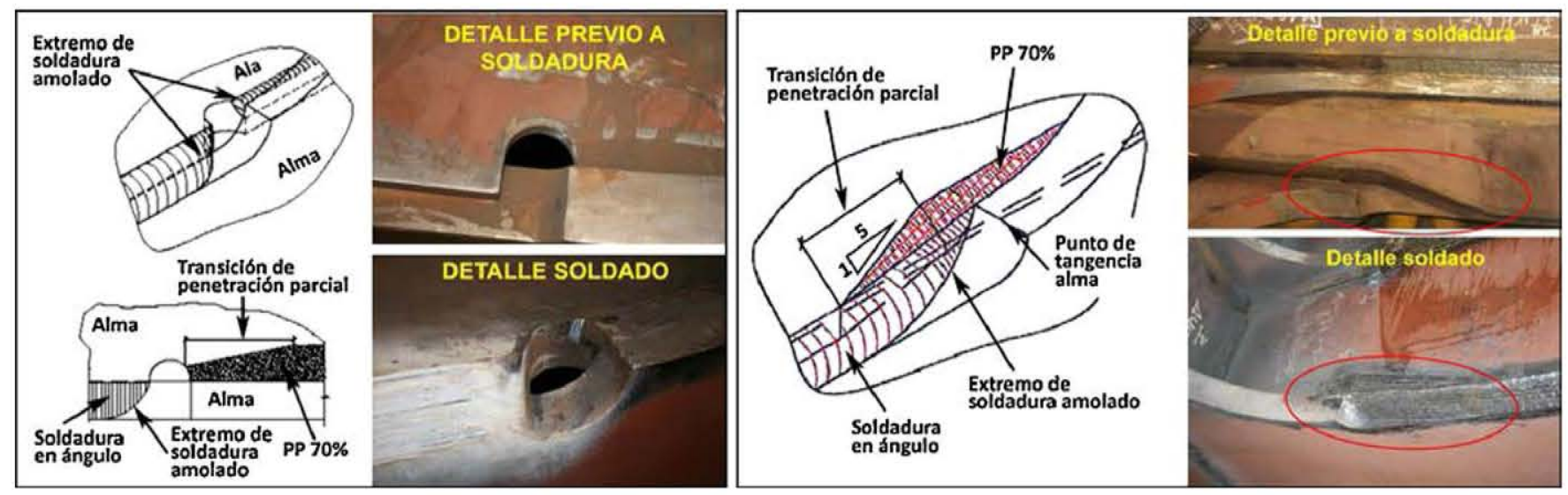

Figura 17. Transición en la zona de inserción de las alas de diagonales o cordones en los nudos. Detalle original con doble ojal (izquierda) y detalle con transición sin ojales (derecha). 


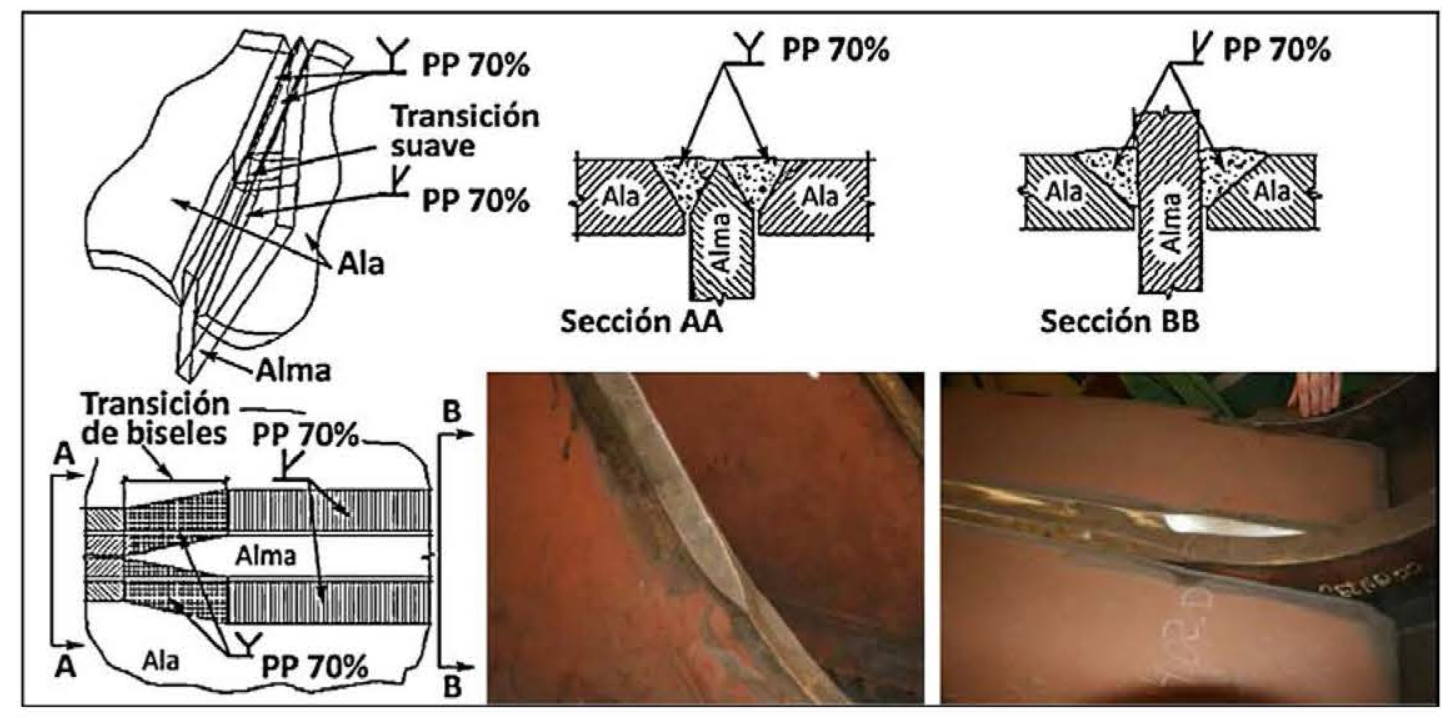

Figura 18. Detalle de transiciones de biseles suavizados en confluencia de alma central en nudos.

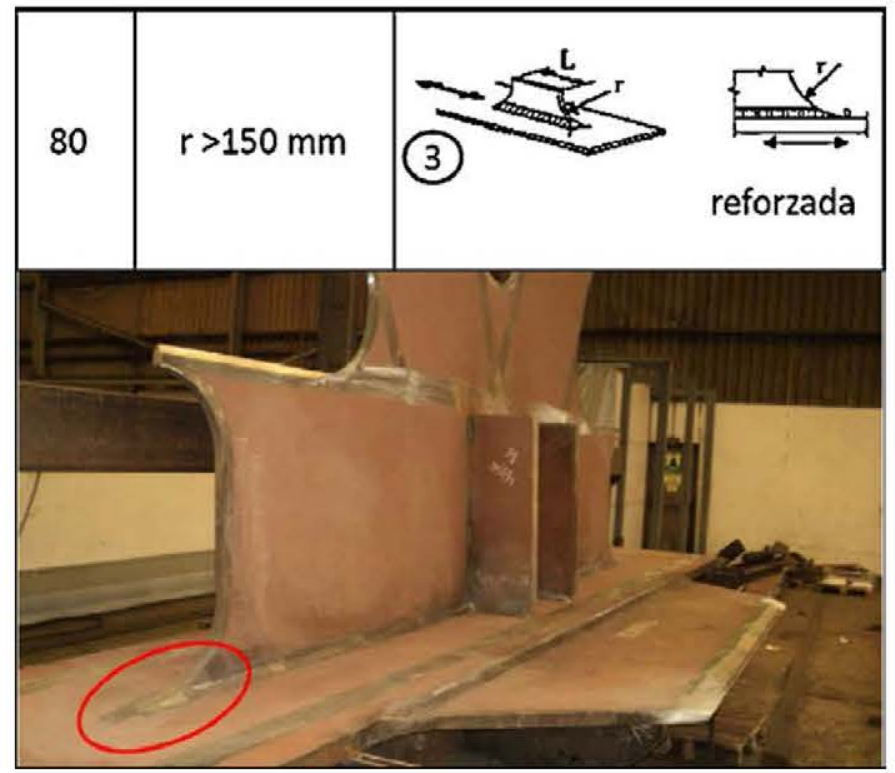

Figura 19. Detalle del extremo del alma central con acabado tangente amolado.

pendiente $1 / 5$ el cambio del bisel, tal y como se define con detalle en cada caso en los planos de taller (fig. 18).

- El extremo de las almas centrales de los nudos debe ejecutarse con un acabado suavizado redondeado con radio superior a $150 \mathrm{~mm}$ y con soldadura amolada tangente, acorde con el detalle 3 de la tabla 8.4 de la referencia [7] con categoría de fatiga 80 (fig. 19).

- La figura 20 muestra el detalle del extremo de soldadura del alma central con el ala superior del nudo inferior, o con el ala inferior del nudo superior, donde la soldadura a penetración parcial del ala con el alma central desmembrada se remata con una transición suavizada con una pendiente $1 / 5$ en un ojal dispuesto al final del alma. El ojal se sella posteriormente con una chapa que garantiza la estanqueidad de los nudos.

- Para arriostrar los cordones superiores de la celosía en todo el tablero y también los cordones inferiores en la zona de canto variable es necesario materializar un plano de arriostramiento mediante cruces de San Andrés con perfiles tubulares que se sueldan a unas cartelas soldadas a los nudos en prolongación de un ala. Esta solución, más o menos convencional en tableros metálicos y mixtos, requiere adoptar las precauciones siguientes para no generar un detalle poco adecuado muy repetitivo:

- La geometría de las cartelas debe acabar con geometría redondeada tangente con radio superior a $150 \mathrm{~mm}$ y soldadura amolada en su extremo, acorde con el detalle 4 de la tabla 8.4 de la referencia [7] para mantener una categoría de fatiga de 90 (fig. 21).

- En general la cartela suele tener siempre un espesor menor que el de las platabandas principales del puente, en este caso de los nudos, y por lo tanto recurrir a la solución más convencional de hacer una transición de espesor en la chapa de mayor espesor, no es la mejor solución, ya que se debilitaría la sección principal resistente longitudinal. En este caso es más conveniente soldar a tope la cartela contra el espesor del ala y suavizar con una soldadura adicional en ángulo que permita una transición más suave de las tensiones de la cartela a la platabanda (fig. 21).

\section{Ejecución del tablero}

\subsection{Ejecución de los vanos de acceso de la margen izquierda mediante lanzamiento}

La ejecución de la estructura metálica de los cuatro vanos de acceso de canto constante de la margen izquierda, de $50+80+120+120 \mathrm{~m}$ (fig. 1), situados entre el E-1 y la P-4, se concibió en la fase de proyecto mediante un proceso de izado por dovelas soldadas sobre apeos provisionales materializando, tras el desapeo, el esquema de viga continua.

Tras la adjudicación de la obra, la UTE río Ulla estudió un proceso de montaje alternativo, adaptando el proceso constructivo original, mediante el lanzamiento de los vanos 3 y 4 (entre 


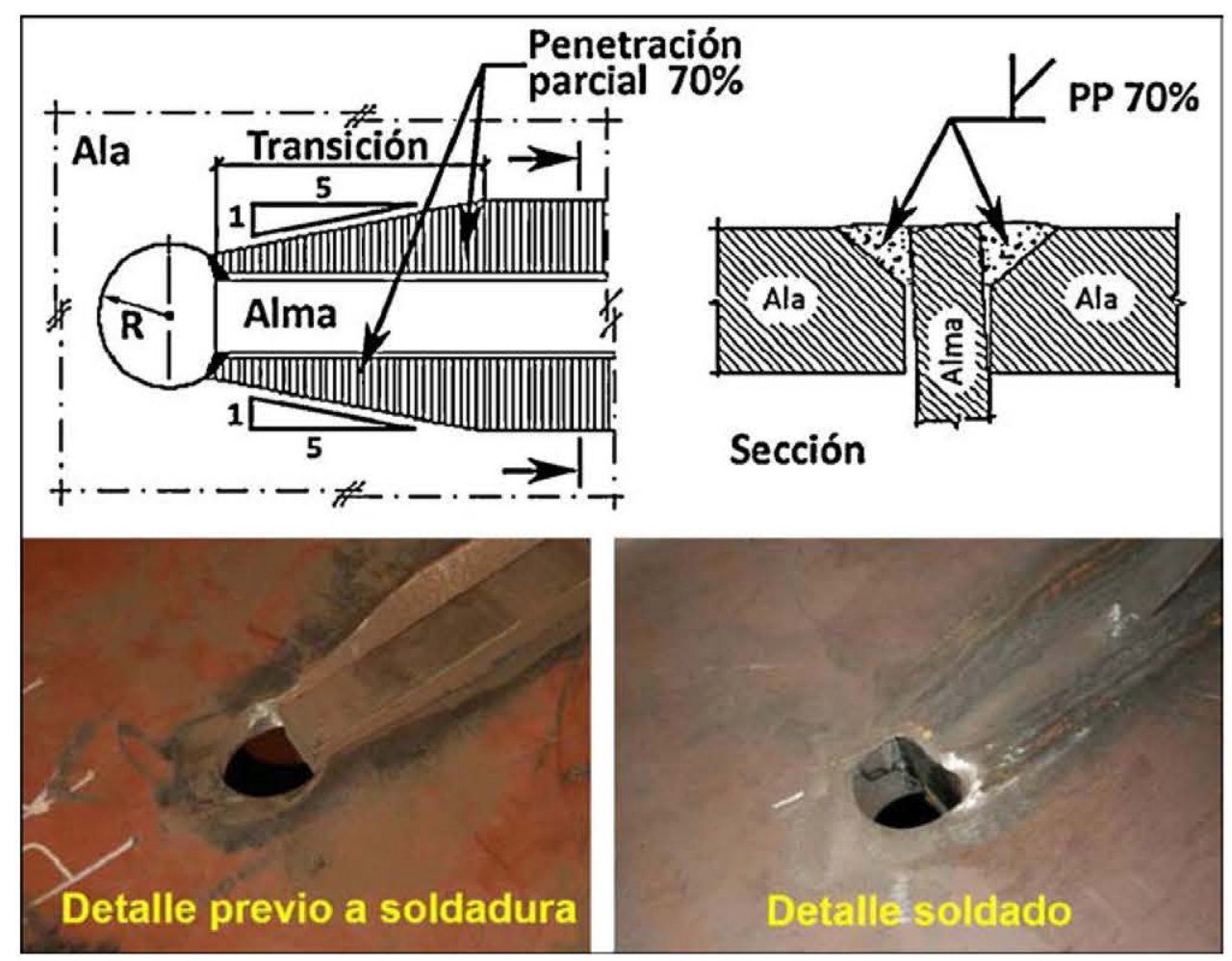

Figura 20. Extremo de alma central en nudos, con ojal en el extremo y transiciones de soldaduras.

las pilas P-2 y P-4), debido a las dificultades de acceso a esas zonas para las dovelas y grúas, junto con las dificultades impuestas por el cruce bajo la traza de dos carreteras entre P-2 y P-3, y de una línea de ferrocarril cercana a la P-4.

Este proceso constructivo respetaba los criterios generales de mantener una estructura resistente en viga continua, empleando la zona ente el E-1 y la P-2 como parque de ensamblaje de dovelas en altura sobre apeos, análogo al montaje originalmente previsto en proyecto, pero evitando los izados entre $\mathrm{P}-2 \mathrm{a}$ P-4.

La cercanía de un túnel tras el E-1, perteneciente además al tramo anterior adjudicado a otra constructora, obligó a materializar el parque de lanzamiento avanzado en altura sobre apeos provisionales y aprovechando las pilas P-1 y P-2 definitivas.
El proceso completo de ejecución de los cuatro vanos se materializó en tres fases, tal y como se representa en el esquema de la figura 22.

En la primera fase se ejecuta en el parque de lanzamiento sobre apeos y las pilas P-1 y P-2, ubicado entre el E-1 y la P-2, el vano 4 de $120 \mathrm{~m}$ de longitud, izando las dovelas mediante grúas. Concluido el tramo, se realiza el primer lanzamiento, avanzando el tramo de $120 \mathrm{~m}$ desde la P-2 hasta la P-3.

El lanzamiento se realiza desde una estructura auxiliar anclada a la P-2, y dado que el tramo presenta una pendiente longitudinal descendente del $-1,8 \%$, el tramo se sujeta de forma continua mediante el empleo de una retenida anclada al E-1. En la fase de lanzamiento se va soltando la retenida y se tira desde los gatos ubicados en $\mathrm{P}-2$.
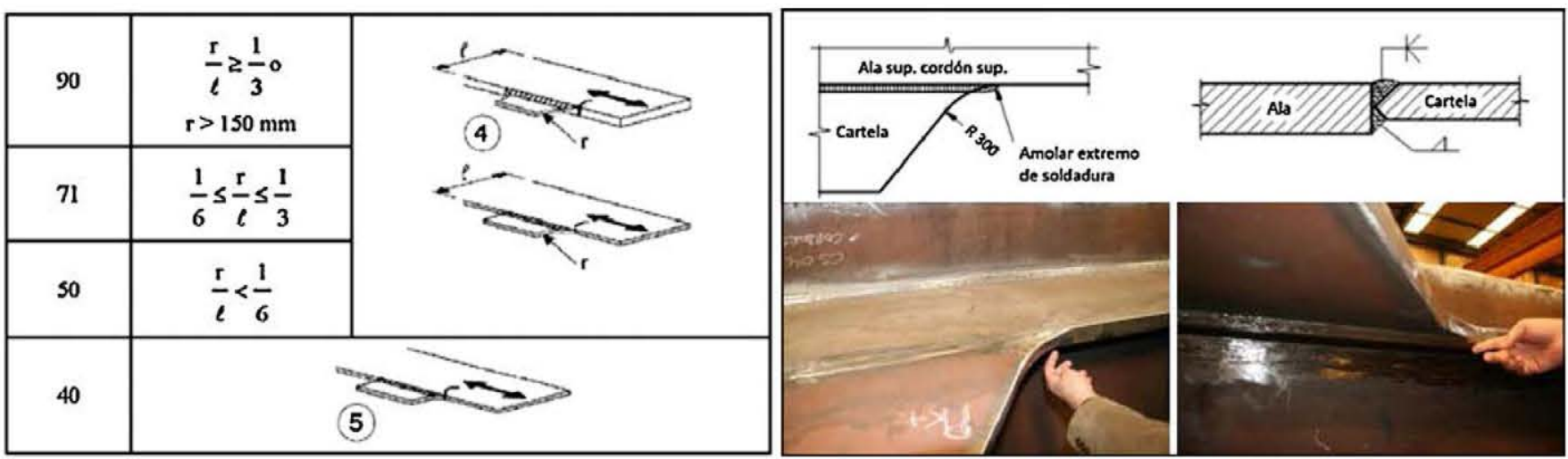

Figura 21. Detalle 4 de la tabla 8.4 de la referencia [7] y vista del extremo de la cartela soldada tangente a la platabanda de un nudo. 


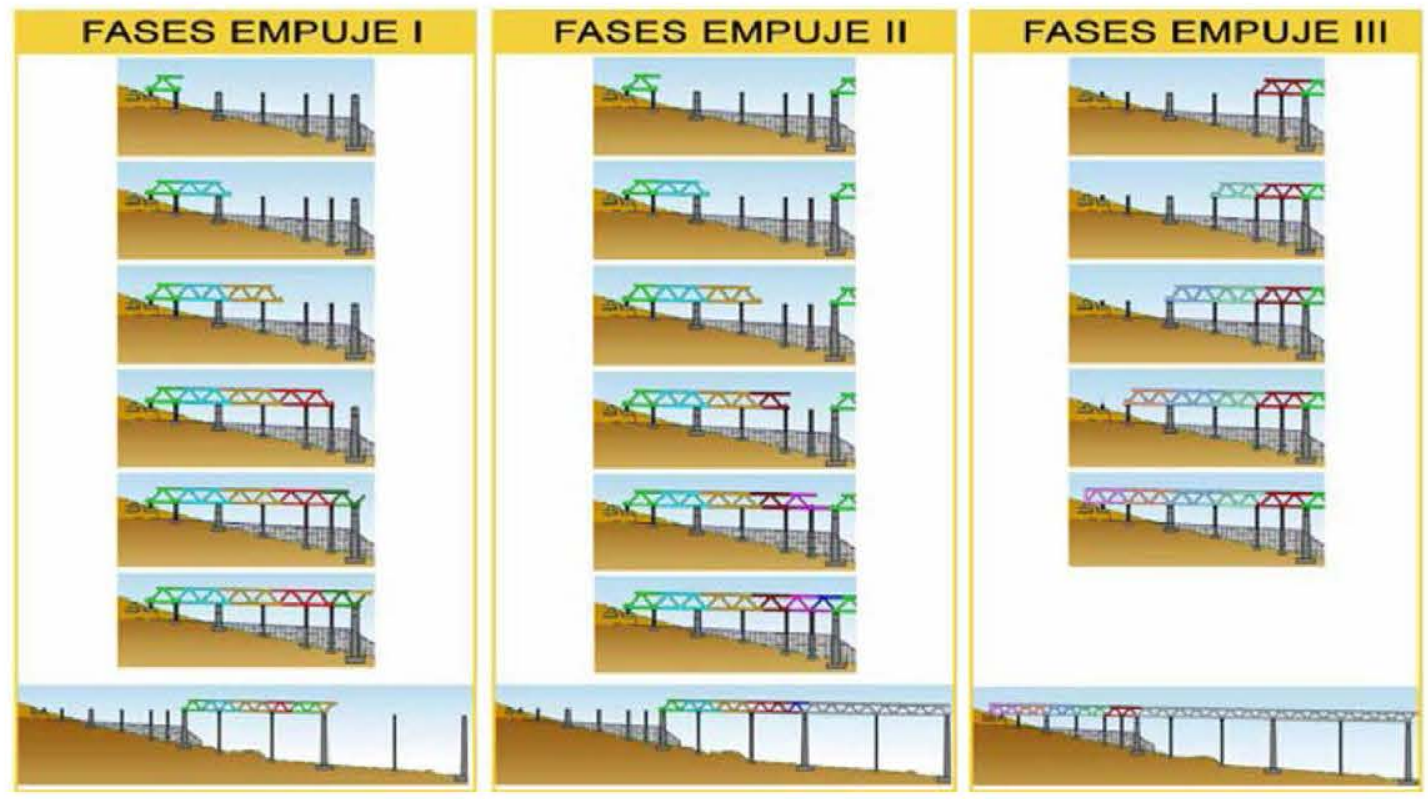

Figura 22. Secuencias del proceso de ejecución y del lanzamiento de los vanos 1 al 4 .

Entre P-2 y P-3 se disponen dos apeos intermedios, que según las fases servirán de apoyo al tramo lanzado, reduciendo la luz del voladizo frontal (fig. 23). Varios de los apeos dispuestos entre el E-1 y la P-2 solo trabajan durante las fases provisionales de ensamblaje y no se emplean durante el empuje.

La segunda fase del proceso de lanzamiento implica el izado de las dovelas del vano 3 en el parque de lanzamiento entre E-1 y P-2 soldado de forma continua con el tramo previamente lanzado. Concluido el tramo, se procede al segundo lanzamiento de los vanos 3 y 4 de $240 \mathrm{~m}$ de longitud, desde la P-3 hasta su llegada a la P-4 $(120 \mathrm{~m})$.

La imagen de la izquierda de la figura 24 muestra el inicio de la segunda fase de lanzamiento saliendo de P-3, y la imagen del centro de la figura 24, el tramo completo ya lanzado entre P-3 y P-4. Entre P-3 y P-4 se dispone de un apeo intermedio, que reduce la luz del voladizo frontal a $60 \mathrm{~m}$. La imagen derecha de la figura 24 muestra la ejecución de la fase 3 (fig. 22) de izado mediante grúas de las dovelas del tramo comprendido entre P-2 y E-1.

La descripción detallada del proceso de lanzamiento, los criterios seguidos en relación a la disposición de gatos en parte de los apoyos provisionales del lanzamiento para controlar directa o indirectamente la reacción en cada apoyo durante todo el proceso, así como las distintas fases intermedias de los dos lanzamientos, junto con los elementos auxiliares necesarios para realizar la operación (gatos y patines abatibles de punta, gatos de cola, orejetas de enganche de las unidades del lanzamiento y de las de retenida), así como el resto de medios auxiliares y de controles realizados durante las dos operaciones, se describen con detalle en la referencia [10].

\subsection{Ejecución de los vanos de acceso de la margen derecha mediante izado}

El tramo de los tres vanos de acceso de canto constante de la margen derecha del río Ulla, comprendido entre las pilas P-9 y el estribo E-2, de $120+120+80 \mathrm{~m}$, respectivamente, se resolvió en la fase de proyecto mediante el izado por dovelas soldadas sobre apeos provisionales materializando, tras el desapeo, el esquema de viga continua.

Entre la pila P-9 y el E-2 no había ningún impedimento ni dificultad inferior, y tras el estudio de la secuencia constructiva, la UTE río Ulla propuso adaptar la secuencia original, ejecutando de forma apeada junto al terreno y con mejor acceso para trabajar a menor altura, los 3 vanos sobre dados de apeo de altura reducida en la sobra del tablero definitivo, para posteriormente
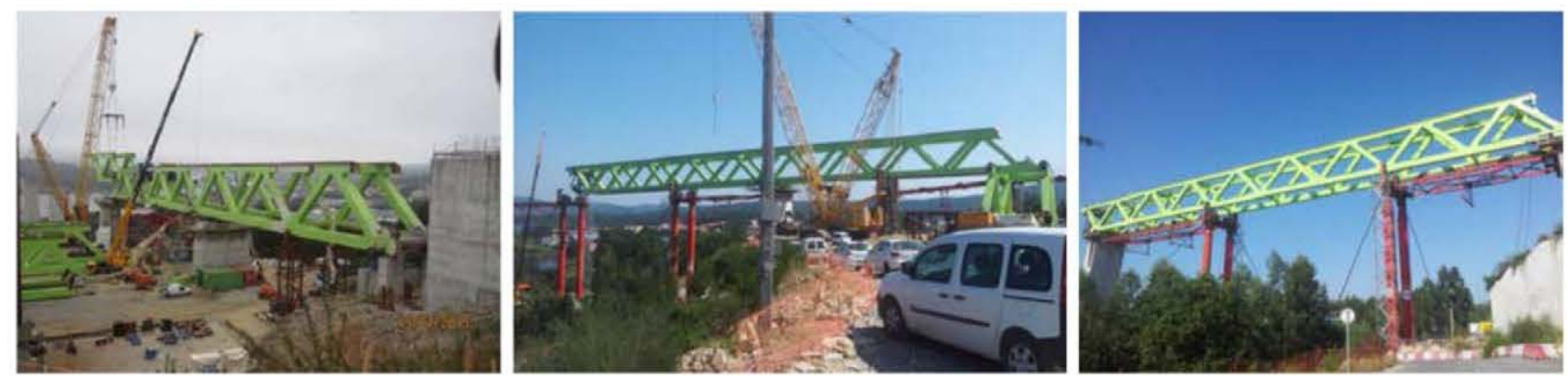

Figura 23. Izado de dovelas, imagen del primer lanzamiento y tramo lanzado entre P-2 y P-3. 

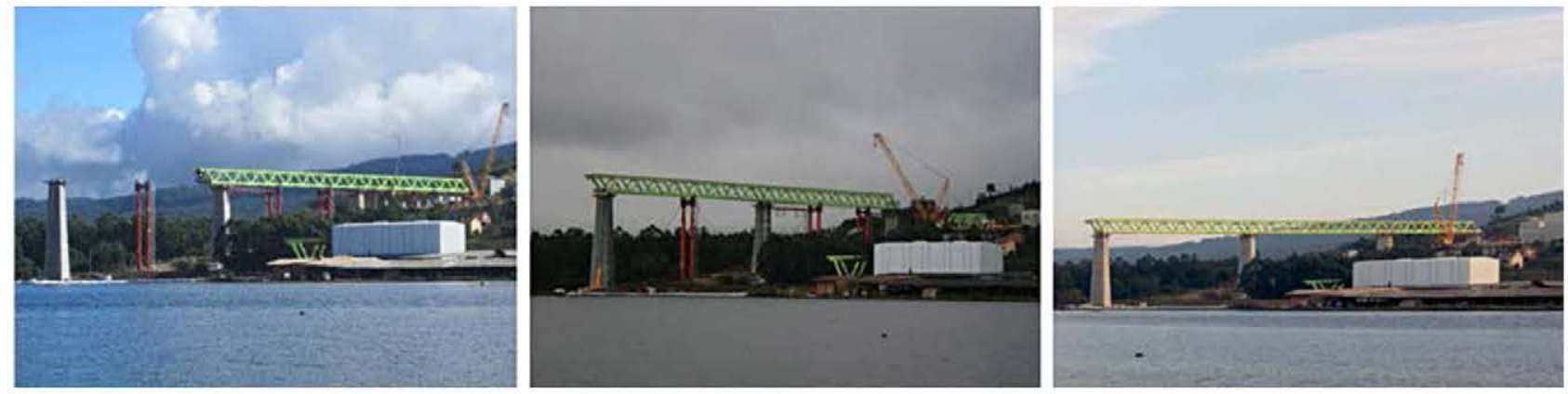

Figura 24. Segunda fase de lanzamiento, tramo de $240 \mathrm{~m}$ ya lanzado entre P-3 y P-4 y ejecución de fase 3.

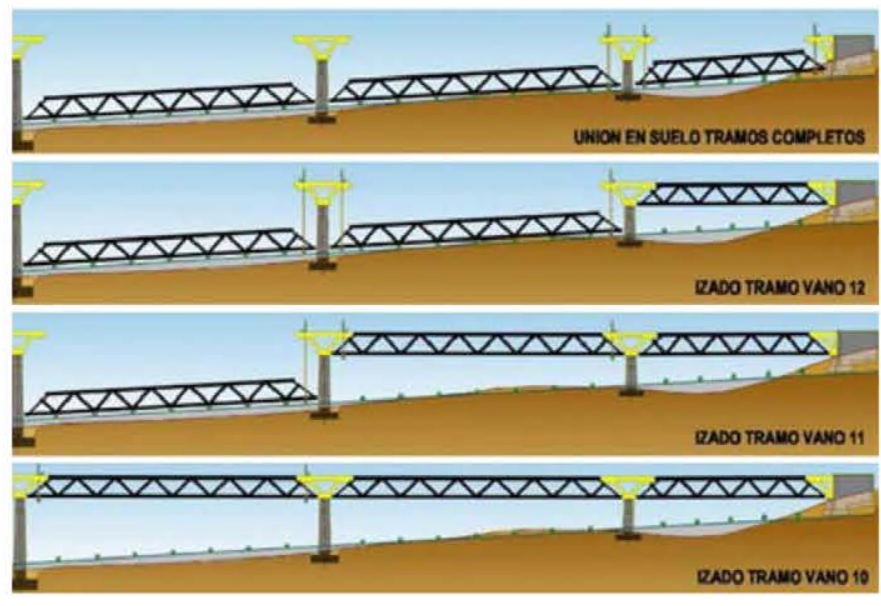

Figura 25. Esquema con la secuencia del proceso de izado de los vanos laterales 12,11 y 10 .

realizar el izado de los tres vanos completos, uno a uno. La secuencia de ejecución e izado se describe en el esquema de la figura 25.

En una primera fase se ejecutaron los tramos a izar apeados junto al terreno (fig. 26), ubicados en posición en la sombra del tablero.

El izado del vano 12 ubicado junto al E-2 (fig. 27), de unas $465 \mathrm{t}$ de peso, se materializó empotrando de forma provisional la dovela cero anclándola al estribo E-2 en su parte superior, y disponiendo unas cajas de arena a modo de tope en su parte inferior, reaccionando contra el estribo [11]. La dovela cero, situada sobre la P-11, al tener forma de delta y encontrarse articulada en su apoyo provisional sobre la pila, se empotró temporalmente, para evitar su vuelco, desde su colocación hasta el instante previo al izado, instante en el que se engancharon los cables de izado al extremo del vano 12 a izar y se sujetaron con los cables de retenida anclados al tramo anterior, de mayor peso, evitando así que pudiera volcar durante el izado, liberando para la operación el empotramiento provisional a torsión.

La operación de izado se realizó controlando la reacción de izado y de la retenida, así como la geometría de la dovela cero.

Una vez izado el primer tramo se fijó provisionalmente el vano en altura a las dovelas cero de sus dos extremos, y con el tramo arriostrado provisionalmente se soldó en posición, una vez que las uniones quedaron correctamente ajustadas. Para cada una de las uniones se diseñaron carretes con demasías en cada extremo de cordón y diagonal para poder ajustar con mayor rapidez y facilidad la unión de las piezas a unir en altura.

Concluido el primer izado y con la dovela cero de P-10 en posición, se procedió a izado del tramo del vano 11 entre P10 y P-11 (imagen izquierda de la fig. 28). Este tramo, de unas $900 \mathrm{t}$ de peso total y $120 \mathrm{~m}$ de longitud entre ejes de apoyos, debe compensarse del lado del vano 10 , con un vano de características y peso similares, por lo que para sujetar provisionalmente de forma segura la dovela cero sobre P-10 fue necesario disponer de un contrapeso que aumentara la seguridad y evitase el posible vuelco de la dovela de cabeza de pila durante la operación de izado.

El tercer y último izado se materializó con el izado del tramo entre P-9 y P10, de unas $965 \mathrm{t}$ de peso, izado desde los dos extremos ya soldados en continuidad con los vanos adyacentes (imagen derecha de la fig. 28).
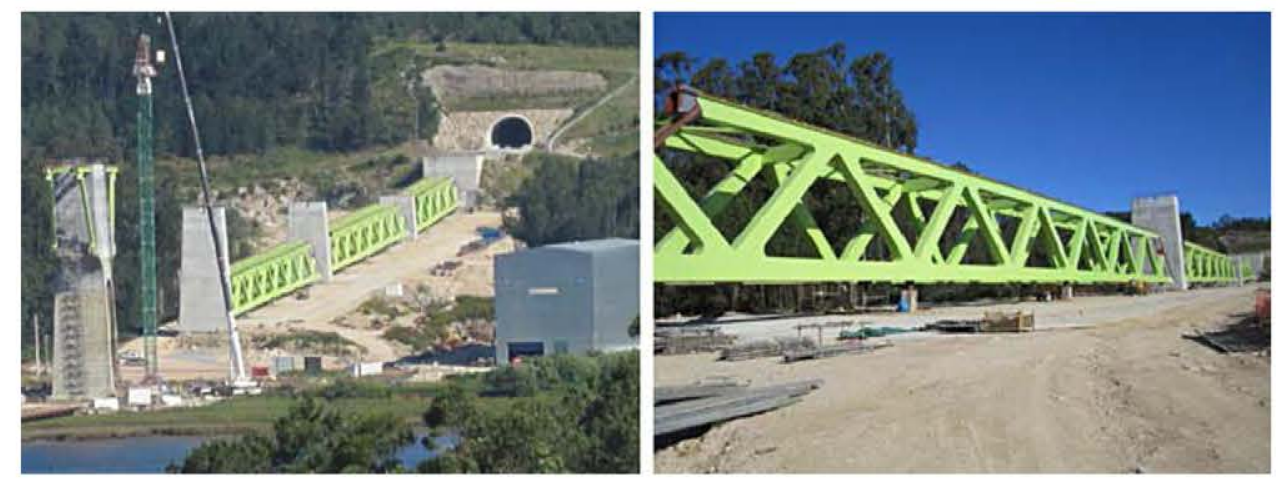

Figura 26. Tramos de los vanos 10 a 12 ejecutados en el suelo preparados para su izado. 


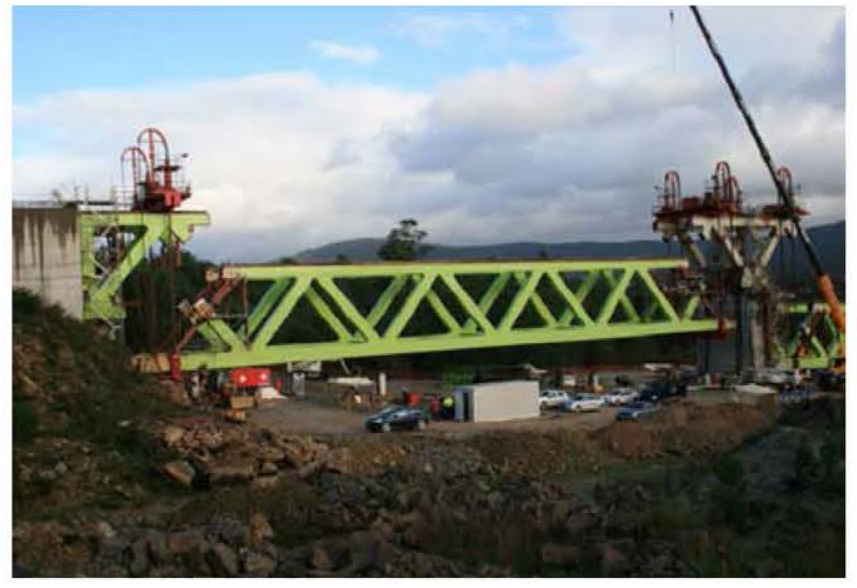

Figura 27. Secuencia del proceso de izado del vano lateral 12 entre el E-2 y la $\mathrm{P}-11$. Izado de unas $465 \mathrm{t}$.

La descripción detallada del proceso de izado de estos tres vanos, así como los elementos auxiliares necesarios para realizar la operación, se describen con más detalle en la referencia [11].

\subsection{Ejecución de los vanos de canto variable por avance en voladizos sucesivos}

El proceso constructivo de los cinco vanos centrales de canto variable de $930 \mathrm{~m}$ de longitud se ha ejecutado, tal y se concibió en proyecto debido a las limitaciones medioambientales impuestas por la ría, mediante el avance por voladizos sucesivos compensados de la estructura metálica desde las cuatro pilas centrales (fig. 29).

La ejecución de la dovela cero metálica con forma de «W» que se queda parcialmente embebida en el cabecero de cada una de las pilas centrales obligó a un complejo proceso de ejecución debido a sus grandes dimensiones y elevado peso. Cada cuchillo de la dovela mide unos $35 \mathrm{~m}$ de longitud, $17,50 \mathrm{~m}$ de altura y pesa unas $375 \mathrm{t}$. En ensamblaje de los elementos simples transportados desde taller se realizó en el pie de cada pila (imagen izquierda de la fig. 30), con cada cuchillo tumbado, aprovechando los recintos de tablestacas creados para la ejecución de las cimentaciones. Una vez soldados todos los elementos, se procedió a realizar la operación de verticalizado simultáneo de los dos cuchillos mediante el empleo de una gran estructura auxiliar provisional dispuesta en cabeza de la pila, con más de $25 \mathrm{~m}$ de altura (imagen derecha de la fig. 30). Esta compleja operación requirió el izado simultáneo mediante gatos por escalones de carga del extremo superior de la dovela y el desplazamiento lateral sobre carriles de los extremos inferiores de las piezas, apoyados de forma articulada sobre patines deslizantes con gatos (fig. 30).

Con los dos cuchillos de la dovela colocados en posición vertical, se procedió al izado compensado de las dos piezas (imagen izquierda de la fig. 31) hasta llegar a la altura de la cabeza de la pila. Para lograr posicionar las dos grandes piezas en su ubicación fue necesario realizar su ripado hacia el interior, el enganche en una articulación inferior, y el posterior giro desde cabeza de cada cuchillo hasta lograr la pendiente de 1/17,5 de la sección transversal. Con los dos cuchillos en su ubicación definitiva, se arriostraron entre sí con los montantes transversales de cabeza y se fijaron en su posición hormigonando los calces inferiores de forma definitiva (imagen derecha de la fig. 31).

Posteriormente se ejecutaron los cabeceros de hormigón, dejando las pilas y la dovela cero listas para iniciar el proceso del avance por voladizos.

Partiendo de la dovela cero, la estructura metálica a ejecutar por avance en voladizos se divide en dovelas de $15 \mathrm{~m}$ de longitud y ancho completo, con un decalaje de 7,5 $\mathrm{m}$ entre el extremo del cordón superior y del cordón inferior, para facilitar las labores de ensamblaje en altura. Desde cada pila se ejecutan las siguientes dovelas por avance en voladizos (fig. 32):

- Desde la pila P-5 se izan 6 dovelas en el vano $5(\mathrm{PK}-)$ y 6 dovelas en el vano $6(\mathrm{PK}+)$.

- Desde la pila P-6 se izan 6 dovelas en el vano $6(\mathrm{PK}-)$ y 6 dovelas en el vano $7(\mathrm{PK}+)$.

- Desde la pila P-7 se izan 7 dovelas en el vano 7 (PK-) y 6 dovelas en el vano $8(\mathrm{PK}+)$.

- Desde la pila P-8 se izan 6 dovelas en el vano 8 (PK-) y 6 dovelas en el vano $9(\mathrm{PK}+)$.

\subsubsection{Ensamblaje y transporte de dovelas de canto variable en obra}

Las dovelas de canto variable se ensamblan en dos de las tres naves de montaje ubicadas junto a las márgenes del río (imágenes
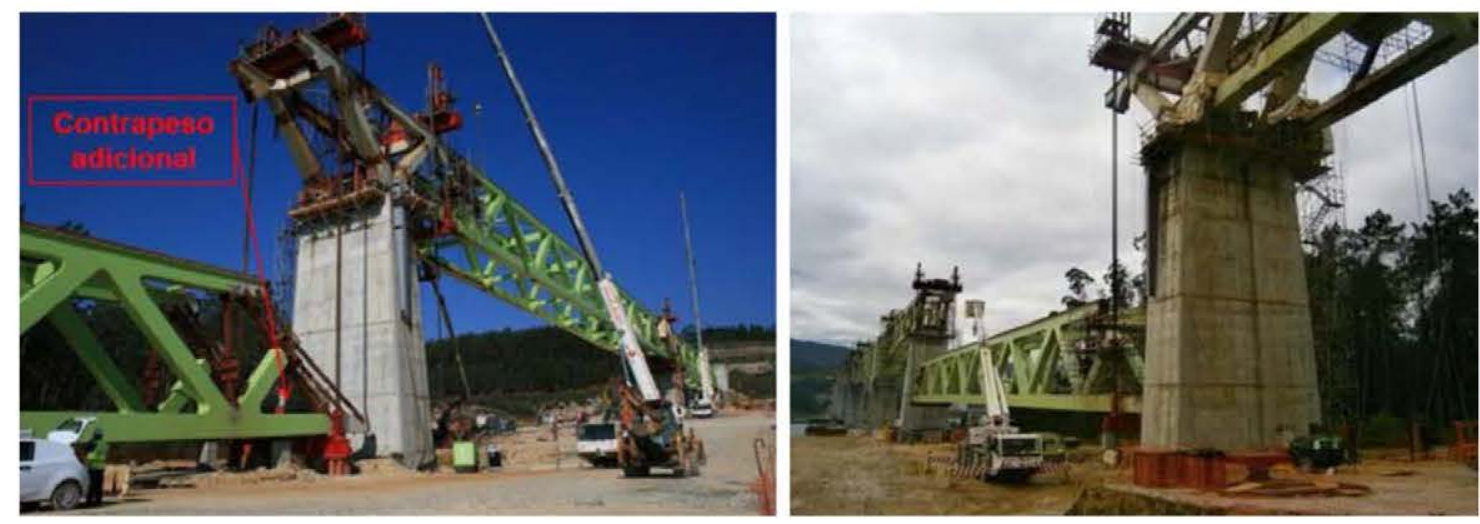

Figura 28. Secuencia del proceso de izado del vano 11 y 10 , respectivamente. 


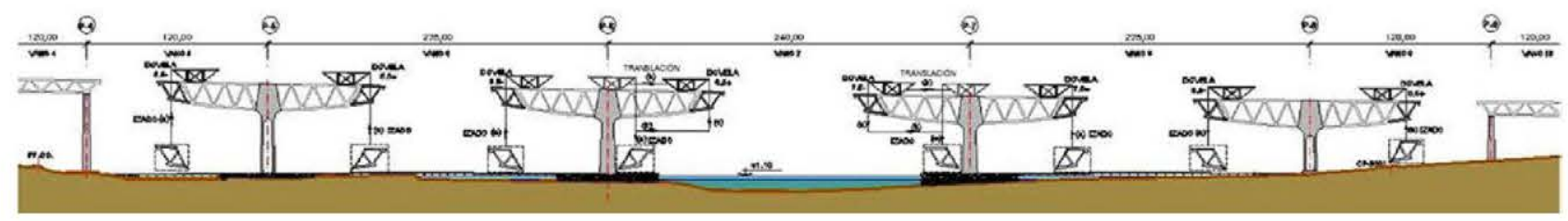

Figura 29. Esquema del avance por voladizos sucesivos en los 5 vanos centrales de canto variable.
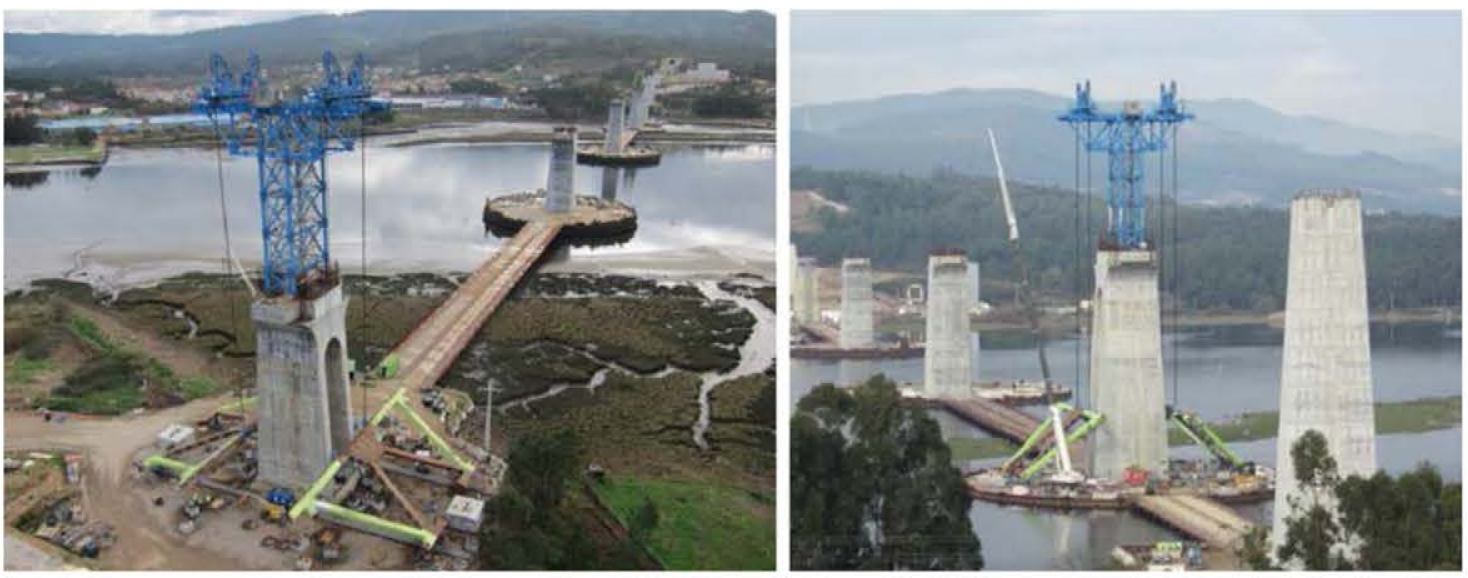

Figura 30. Ejecución de dovela cero en el pie de pila y operación de verticalizado.
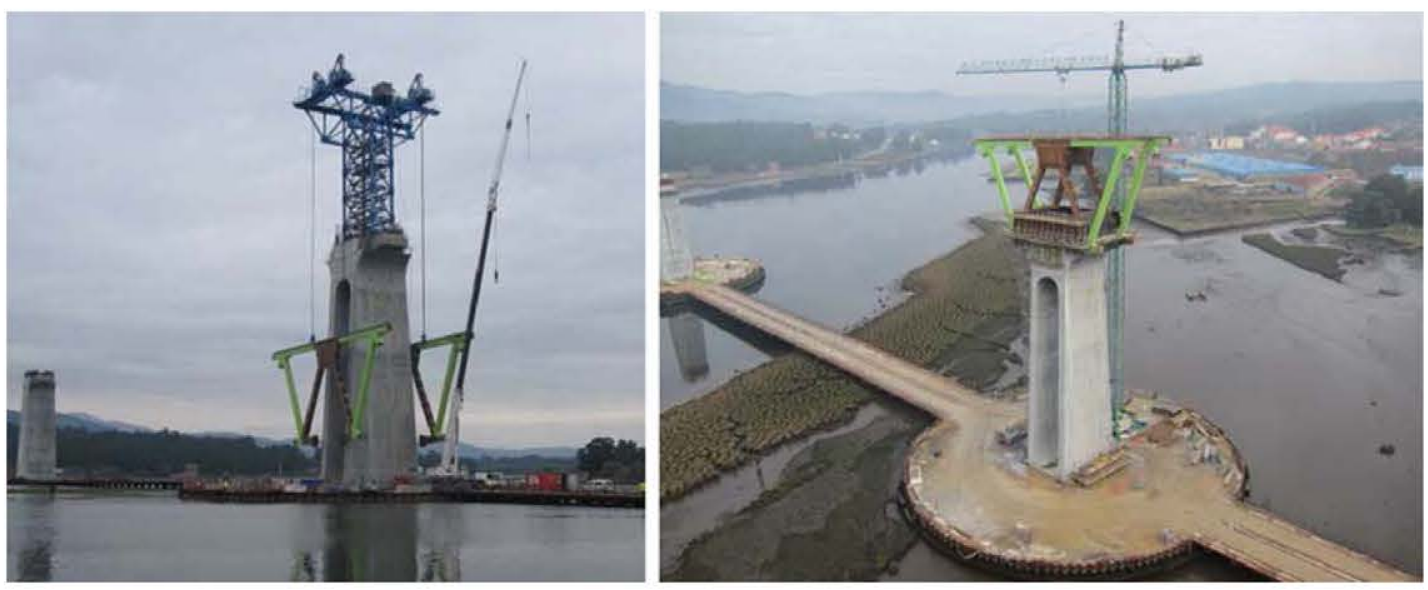

Figura 31. Izado de la dovela cero y ejecución de la cabeza de pila.

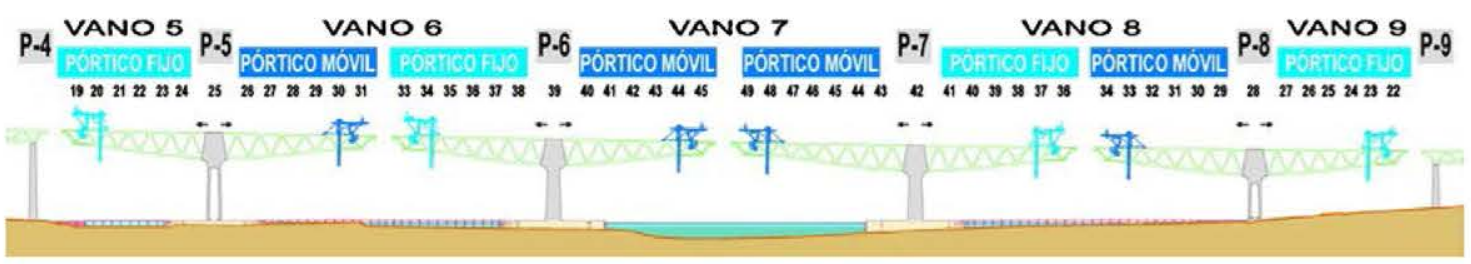

Figura 32. Esquema de división en dovelas de los 5 vanos de canto variable y carros de izado.

central y derecha de la fig. 14). Las dovelas tienen una altura máxima próxima a los $17,50 \mathrm{~m}, 7,10 \mathrm{~m}$ de ancho en la parte superior, casi $10 \mathrm{~m}$ en la parte inferior, $22,5 \mathrm{~m}$ de longitud total desde el extremo frontal del cordón superior al extremo trasero del cordón inferior y un peso máximo de hasta casi $300 \mathrm{t}$.

Dadas las grandes dimensiones de las dovelas, por motivos constructivos y de transporte las dovelas de canto variable se descomponen en los elementos simples siguientes: cordón superior trasero en voladizo, nudo superior frontal, diagonal frontal y diagonal trasera en voladizo, nudo inferior y cordón inferior en voladizo, junto con los montantes y cruces de arriostramiento de nudos y cordones superiores e inferiores (fig. 33).

El ensamblaje de dovelas se realiza en cadena con dovelas ejecutadas por pares, de dos en dos, con montajes en blanco 

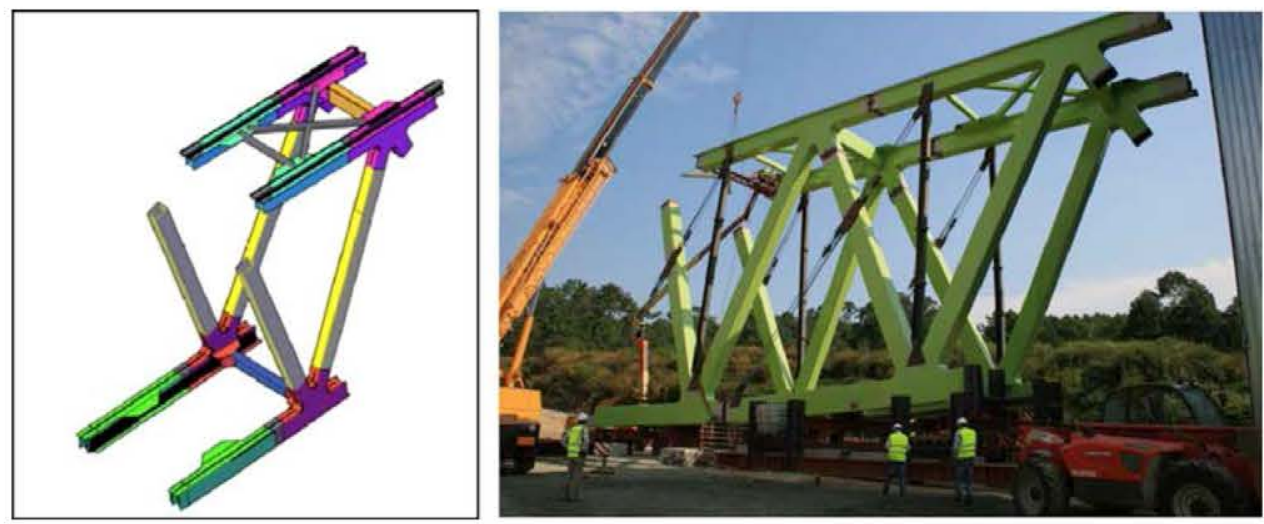

Figura 33. Despiece de elementos de una dovela e imagen de 2 dovelas montadas en blanco.

físicos (fig. 33), de forma que se pueda asegurar el perfecto ajuste en altura de sus 6 extremos. En los casos que no se han realizado montajes en blanco físicos se ha realizado un montaje en blanco virtual por ordenador, lo cual ha permitido asegurar la misma precisión y garantía que con los montajes en blanco físicos.

El diseño de las dovelas «abiertas» con el nudo superior decalado respecto del nudo inferior requiere el arriostramiento provisional reutilizable [12] de los extremos traseros del cordón superior en voladizo, la diagonal volada que parte del nudo inferior y del cordón inferior también en voladizo, que se ha decalado 7,5 m respecto del superior (fig. 33), permitiendo controlar las deformaciones durante el transporte e izado, así como la regulación de la posición final de los extremos de la diagonal y cordón inferior traseros en voladizo a la hora de realizar el ajuste con la dovela precedente.

El transporte de las dovelas de canto variable dentro de la obra se realiza mediante el empleo de plataformas autopropulsadas con dos carros independientes (uno por cuchillo de la dovela) con múltiples ejes tractores (fig. 34).

El vano 9 tiene acceso directo por tierra, y el pantalán permite el transporte e izado vertical de todas las dovelas de los vanos 5, 6 y 8 (fig. 29). El vano central entre P-6 y P-7 es el único tramo ejecutado por avance en voladizos al que no hay acceso por tierra.

Durante la fase de proyecto y al inicio de las obras se estudió la posibilidad de realizar el transporte de las dovelas de los dos brazos del vano 7 mediante el empleo de barcazas, pero las dificultades técnicas de esa operación recomendaron desechar este proceso. El izado de las dovelas del tramo central sobre la ría se ha resuelto mediante el empleo de carros de izado móviles, como se describe más adelante.

Mediante este proceso constructivo, los vanos de canto variable se independizan del terreno inferior sin afectar al cauce principal, a las marismas ni a la vegetación de ribera, condicionantes de obligado cumplimiento a respetar desde las bases del concurso del proyecto.

\subsubsection{Carros de izado de dovelas}

La ejecución del tablero se realiza mediante el avance compensado por voladizos primero desde $\mathrm{P}-5$ y $\mathrm{P}-8$, y posteriormente desde las pilas centrales P-6 y P-7, para lo cual ha sido necesario diseñar y fabricar cuatro carros de izado - dos de ellos fijos y otros dos móviles - sobre los que se dispone un sistema de izado con dos gatos hidráulicos.

Los carros fijos se denominan así porque el izado de una dovela se realiza en posición fija anclada al tablero (fig. 35) y, por lo tanto, su movimiento de avance se realiza con el carro sin carga, soportando solo su peso propio.

Los carros móviles (fig. 36) se denominan así porque son capaces de recoger una dovela en la base de la pila, izarla parcialmente, trasladarla por el aire (bajo el voladizo existente) hasta su ubicación final en el extremo de la ménsula e izarla hasta su posición final para el soldeo con la anterior. Para la ejecución del tramo central entre P-6 y P-7 sobre el agua son necesarios dos carros móviles, motivo por el cual se han diseñado y fabricado dos carros fijos y dos móviles.

El avance por voladizos desde las pilas P-5 y P-8, al existir acceso en todos los tramos por tierra o por el pantalán provisional, los carros móviles funcionan como carros fijos, izando todas las dovelas en vertical con los carros anclados al tablero, sin necesidad de realizar la compleja operación de traslación de una dovela parcialmente izada.

El desplazamiento de los carros sobre la celosía se realiza por medio de patines de deslizamiento que deslizan, mediante el empleo de gatos hidráulicos, sobre unos carriles con apoyos de neopreno-teflón lubricados dispuestos sobre las cabezas de los cordones superiores de la celosía.

Los carros fijos disponen los gatos de izado dispuestos en la vertical sobre los cuchillos de la dovela (fig. 35), donde se colocan las orejetas de izado, coincidiendo sensiblemente con la posición del centro de gravedad de la dovela. Para permitir el posterior ajuste en posición de la dovela, una vez izada, se ha diseñado un balancín con un gato inclinado (fig. 35), enganchado a las orejetas de ajuste, dispuestas en posición más avanzada, de forma que cuando el gato de ajuste se abre, la dovela pivota acercando el cordón inferior a su posición, y si el gato cierra el pistón, pivota en sentido contrario, alejando la posición del cordón inferior [12].

La principal diferencia de los carros móviles con los fijos es que, para permitir el transporte de las dovelas parcialmente izadas, los gatos y cables de izado no pueden disponerse en la misma vertical que las orejetas de enganche en la dovela, dispuestas sobre los cordones superiores, haciendo necesario 

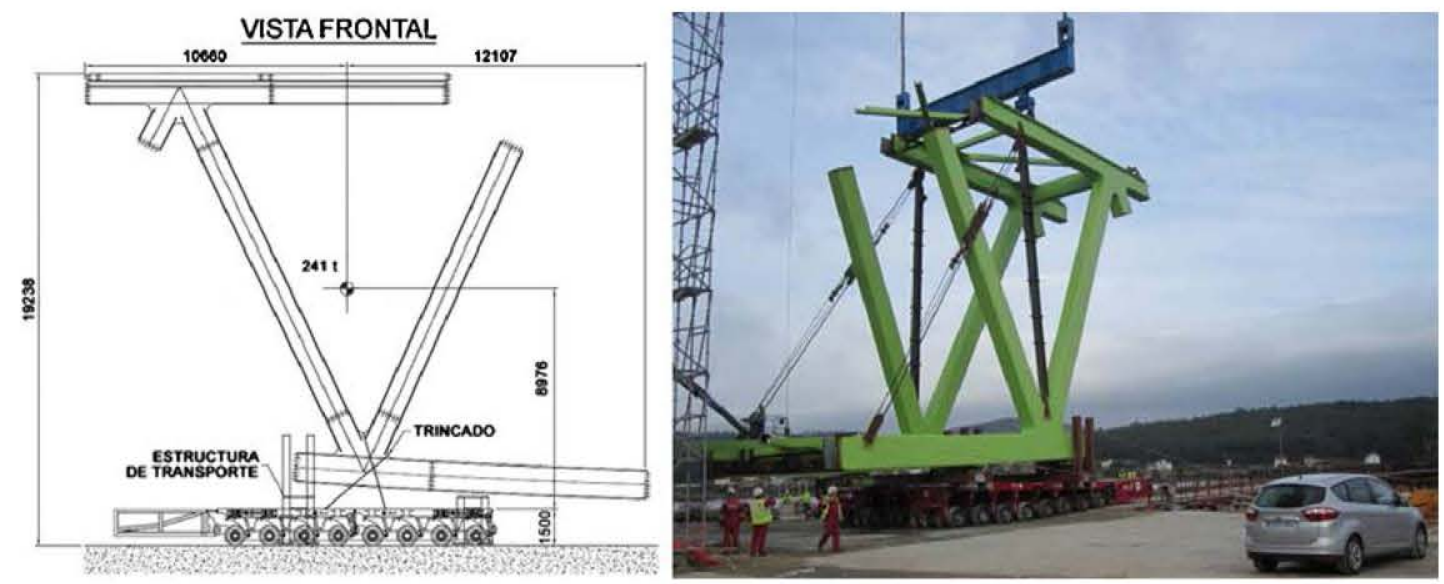

Figura 34. Transporte de dovelas con plataformas autopropulsadas (esquema e imagen).

abrirlos por fuera sobre una viga transversal ubicada sobre el carro y disponer de una viga balancín transversal de enganche entre la dovela y los cables de izado (fig. 36). Para permitir el correcto ajuste en posición de la dovela, una vez que se sitúa en cota, se dispone de cables de enganche a unas orejetas ubicadas en el extremo de la dovela que permiten corregir ligeramente la posición para el soldeo [12].

\subsubsection{Ajuste en posición de la dovela y soldadura en altura}

Cuando las dovelas se aproximan a su posición, se verifica mediante topografia su correcto posicionamiento en su ubicación definitiva, para proceder a soldarla en altura con la precedente. Durante el proceso de posicionamiento se actúa sobre los gatos de izado y el balancín de ajuste (en los carros fijos) o sobre los gatos de tensión de los cables de rigidización (en los carros móviles). Además se utilizan, en los casos necesarios, trácteles o gatos manuales de aproximación.

Tanto los trabajos de acople como los trabajos de soldadura en altura se realizan desde plataformas de soldeo protegidas del aire y la lluvia diseñadas específicamente para este fin. Cada carro dispone de cuatro plataformas de soldeo: dos delanteras y dos traseras. Las plataformas delanteras habilitan el acceso para el acople y soldadura de los cordones superiores, diagonales y arriostramientos superiores (fig. 37). Por su parte, las plataformas traseras disponen de movilidad longitudinal y regulación en altura, facilitando el acceso a los cordones y arriostramientos inferiores a distinta cota según varía el canto del tablero.

Las plataformas delanteras de soldeo disponen de partes desmontables interiores que deben ser montadas para acceder a la cara interior de los cordones y desmontadas para posibilitar el avance del pórtico. Las plataformas de soldeo traseras sirven a su vez para la retirada de la rigidización provisional durante el avance de los pórticos.

A grandes rasgos, la secuencia del proceso una vez posicionada la dovela izada es la siguiente: montaje de las partes interiores de las plataformas delanteras, acople y soldadura del cordón superior y el arriostramiento superior en cruz de San Andrés, acople y soldadura del cordón inferior y diagonal, cambio de posición de plataformas de soldeo traseras, acople y soldadura del arriostramiento inferior en cruz de San Andrés, soldeo de la cabeza superior del cordón superior, desmontaje parcial de las plataformas de soldeo delanteras y, finalmente,
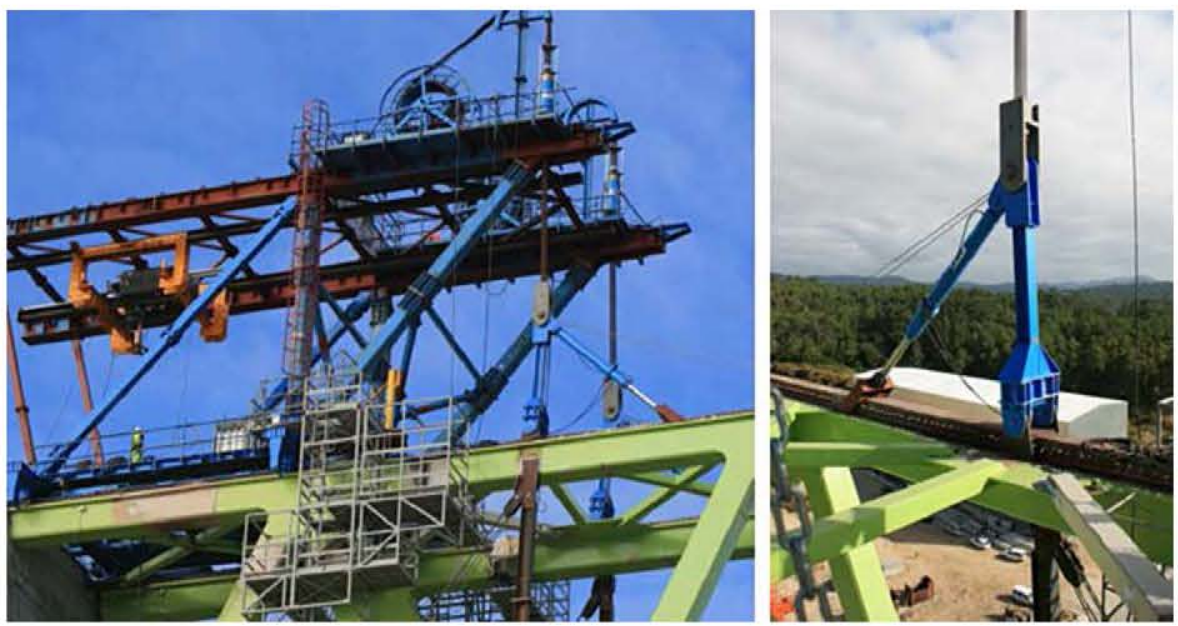

Figura 35. Izado de una dovela desde carro fijo y detalles de balancín de ajuste. 

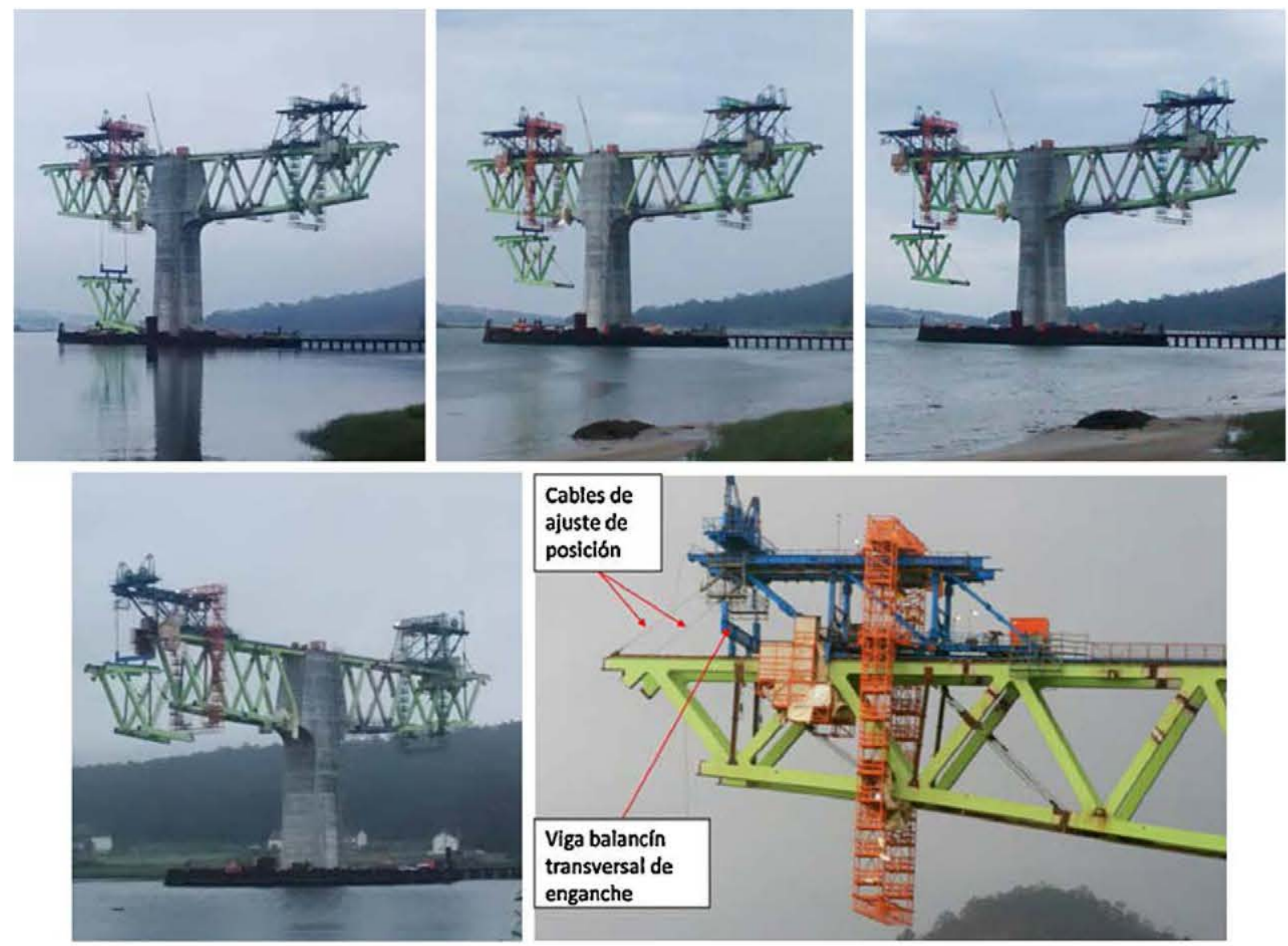

Figura 36. Secuencia de enganche, izado y traslación de una dovela con carro móvil. Izado final en poisción y detalles de viga balancín y cables de ajuste de posición de la dovela.

desenganche de la dovela izada y avance del carro para nuevo izado.

Las imágenes siguientes muestran en fotografias el avance en voladizos sucesivos realizado desde P-5 (fig. 38), así como varias fases del avance en voladizos desde el resto de pilas (fig. 39).

\subsubsection{Cierres del avance en voladizos}

Los cierres de los vanos de canto variable se ejecutan, salvo en el caso del vano 9, mediante una dovela con geometría especial. La dovela se divide en dos partes: el cordón superior, que se suelda a los extremos de los dos nudos adyacentes de ambos voladizos, y la parte inferior de la dovela, compuesta por dos
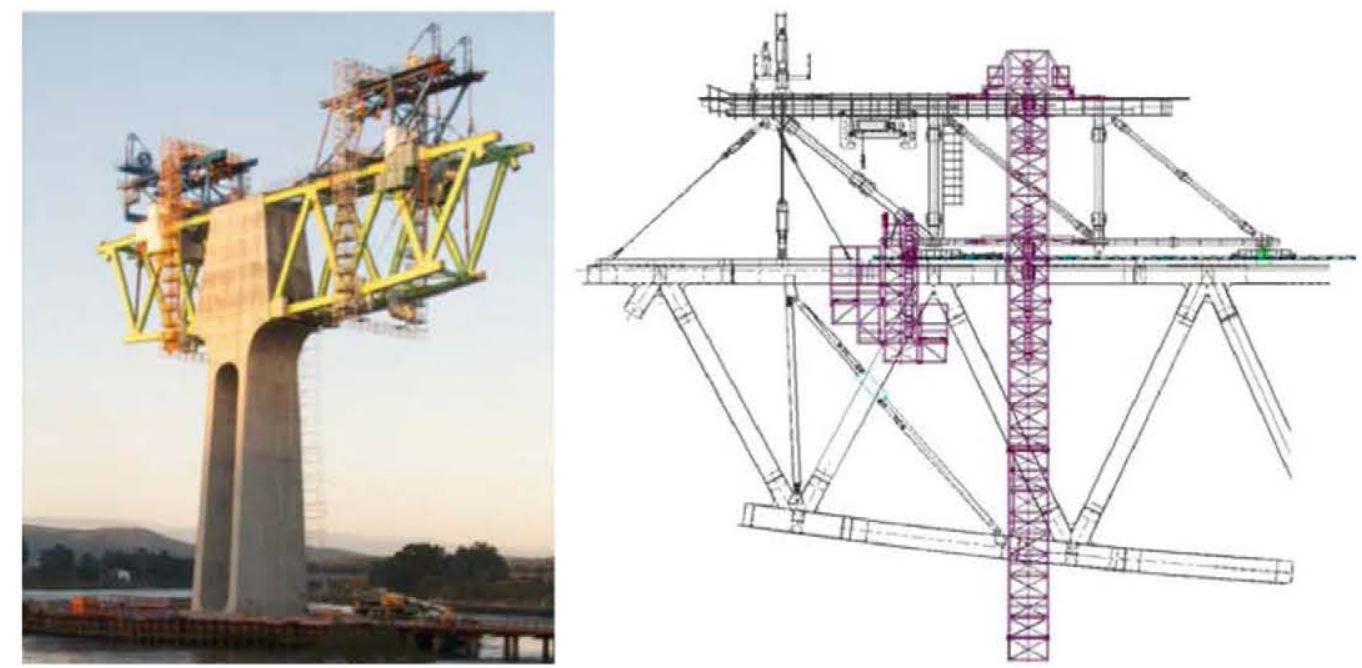

Figura 37. Vista y esquema de plataformas de acceso con zonas protegidas para el soldeo. 

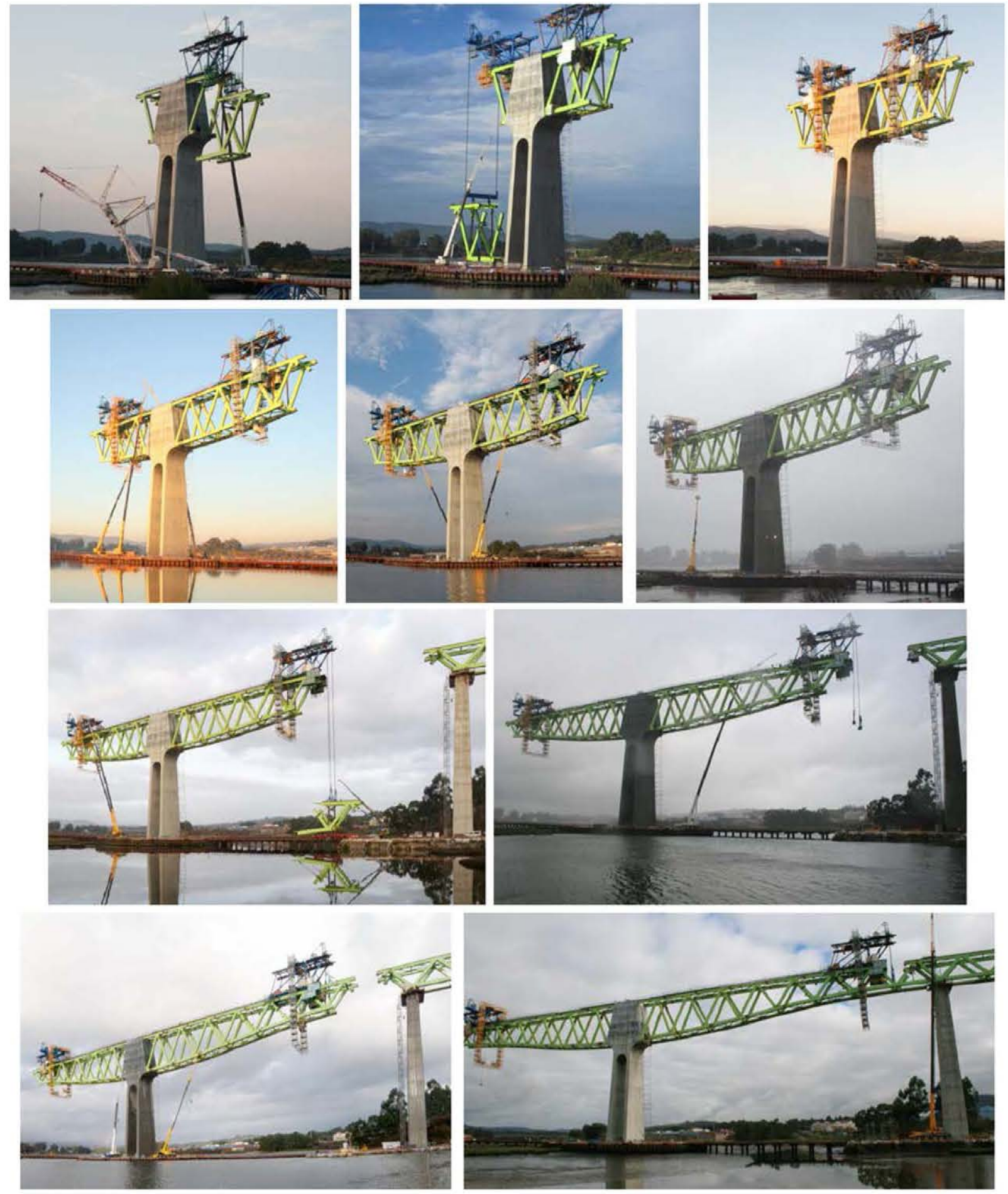

Figura 38. Diferentes vistas del avance por voladizos sucesivos compensados. Pila 5 en primer plano.

cordones inferiores, el nudo central inferior y dos diagonales en voladizo partiendo del nudo inferior.

El proceso de ejecución que se siguió en todos los casos fue el siguiente:

- Izado de la parte inferior de la dovela y ajuste en posición de uno de los dos extremos. En el otro extremo la unión se había diseñado con un carrete en el cordón inferior y en la diagonal para poder ajustar con precisión la longitud.

- Fijación provisional con elementos auxiliares de trincado en el extremo opuesto del cordón y diagonal para posteriormente ajustar la dimensión del carrete de cierre y proceder a su colocación y soldeo.

- De forma simultánea y en horario nocturno, se fija mediante útiles provisionales el extremo de los dos nudos superiores, 

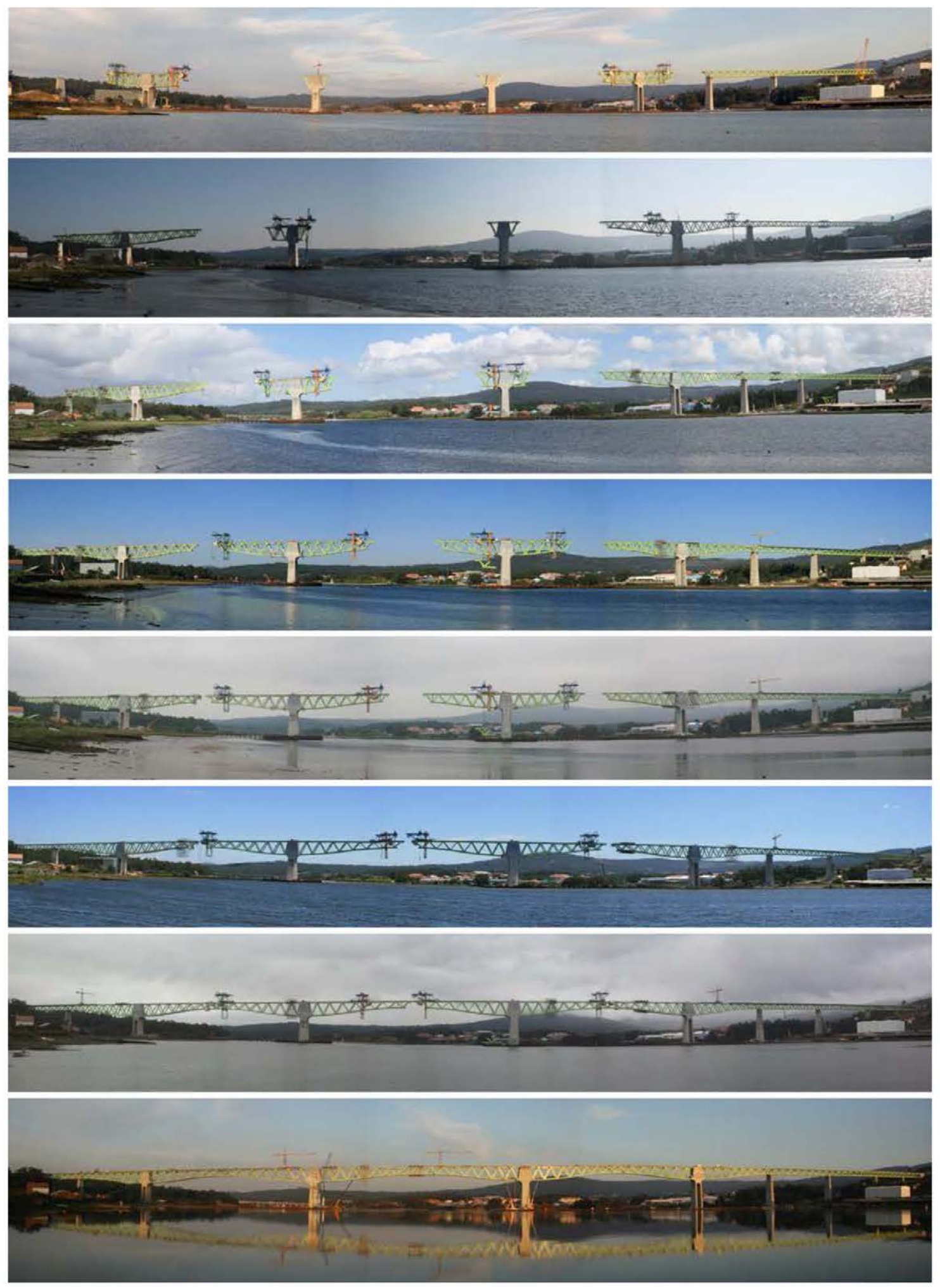

Figura 39. Avance por voladizos sucesivos desde P-5 (a la derecha) y P-8(a la izquierda) y posteriormente desde P-6 y P-7.

para posteriormente colocar el cordón superior de cierre con la ayuda de carretes de ajuste en ambos extremos.

- Una vez soldados y ensayados todos los elementos, se retiran los útiles de trincado auxiliares y se suelta la carga de los carros de izado, dejando el vano en continuidad.
El orden de las operaciones de cierre ha sido el siguiente:

- Cierre del vano 5, junto a P-4, izando la dovela desde el carro fijo del extremo del voladizo de PK- (fig. 40). 

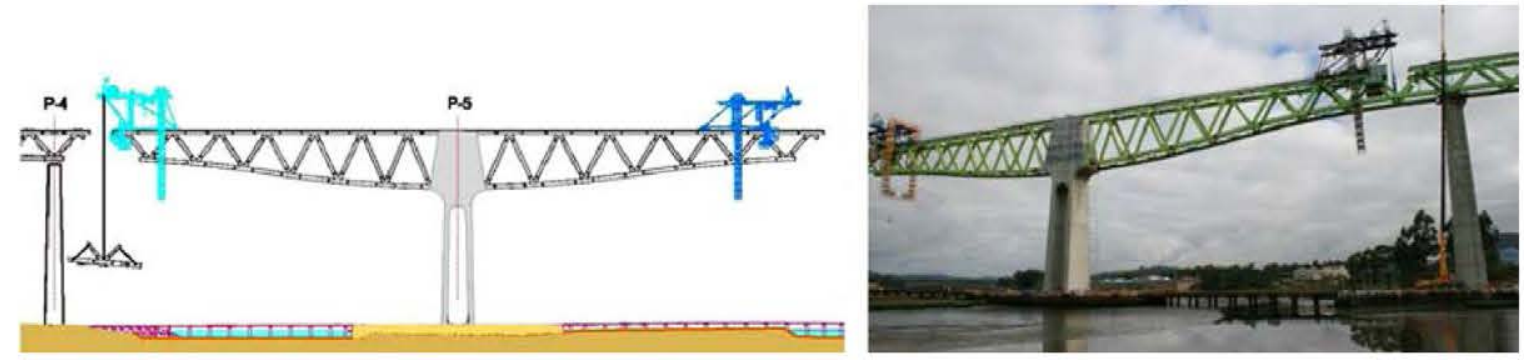

Figura 40. Cierre del vano 5 desde carro fijo.

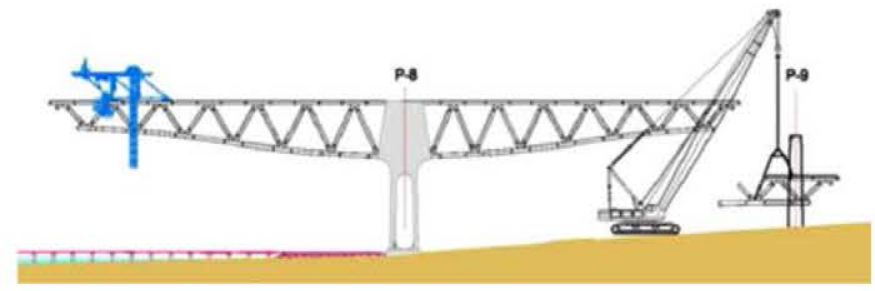

Figura 41. Cierre del vano 5 desde carro fijo, y cierre del vano 9 desde tierra con grúa.

- Cierre del vano 9. Izado desde tierra de una dovela doble mediante el empleo de grúas, dejando la dovela apoyada en la P-9 y soldando el otro extremo al voladizo de PK+ (fig. 41). Desde el extremo de la dovela situada sobre pila es desde donde se izó el vano 10 completo (imagen derecha de la fig. 28).

- Cierre del vano 6, izado de la dovela desde el carro fijo del extremo del voladizo de PK- de P-6 (fig. 42).

- Cierre del vano 8, izado de la dovela desde el carro fijo del extremo del voladizo de PK+ de P-7 (fig. 43).

- Cierre del vano central de $240 \mathrm{~m}$ de luz (vano 7) desde el carro móvil del extremo del voladizo de $\mathrm{PK}+$ de P-6 (fig. 44).

\subsubsection{Ejecución del hormigón de fondo y de la losa superior}

Una vez realizados los cierres del vano 5 y del vano 9 con los vanos laterales en continuidad desde el E-1 hasta P-5 y desde el E-2 hasta P-8, se ha procedido a realizar un descenso simultáneo y controlado de $25 \mathrm{~cm}$ de los apoyos de las pilas P-4 y P-9, con objeto de reducir las flexiones en las pilas principales adyacentes P-5 y P-8 por efecto pórtico, al tener vanos muy descompensados

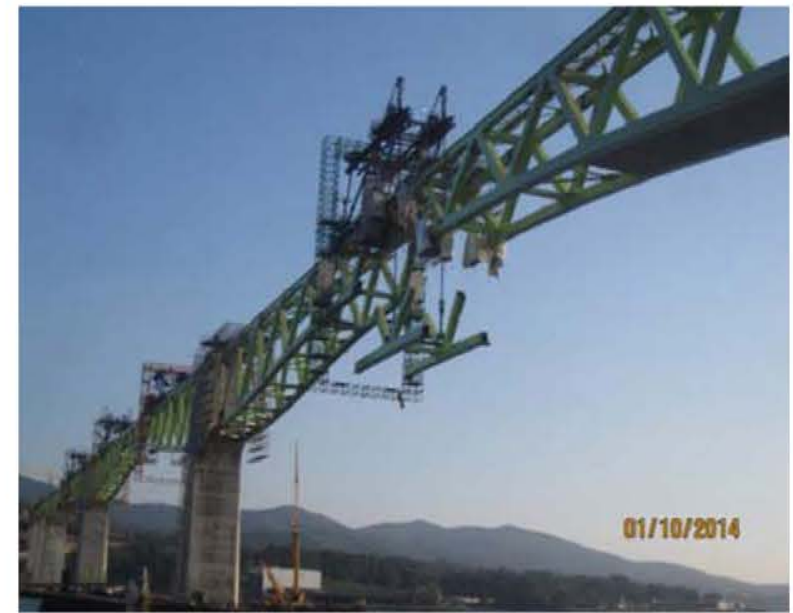

Figura 43. Izado de dovela de cierre del vano 8.

a ambos lados (225 y $120 \mathrm{~m}$ de luz). Este descenso de apoyos centra los momentos flectores de cargas permanentes en dichas pilas.

Con un cierto decalaje respecto de la ejecución de la estructura metálica, se procede a realizar el hormigonado inferior del tablero en todo el puente, estructural y colaborante en zonas de flexión negativa y a modo de mero cierre formal en zonas de flexión positiva sin conexión con la celosía para hacer la sección interiormente visitable e inspeccionable. El hormigonado de fondo se ejecuta mediante el empleo de un carro de encofrado (fig. 45) que se va desplazando por la zona de canto constante y de canto variable del viaducto, colgado de una estructura auxiliar superior que se apoya en las cabezas de los cordones superiores.

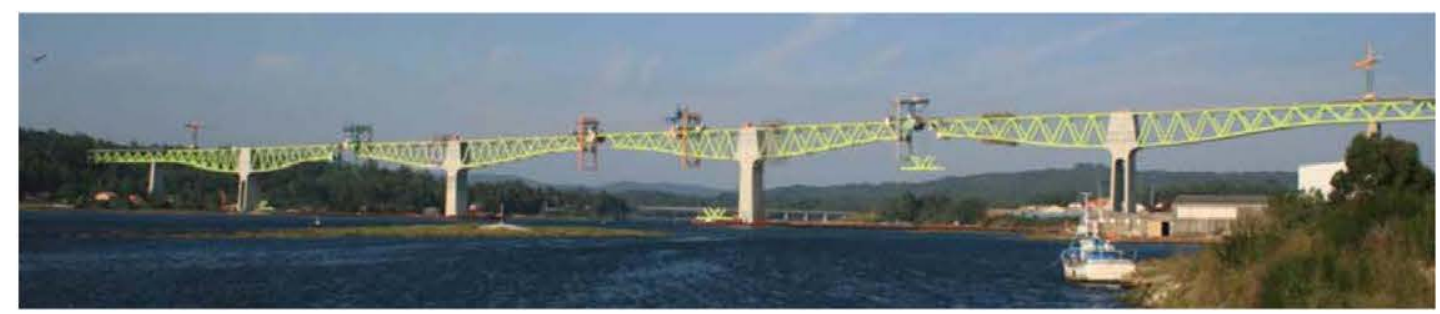

Figura 42. Izado de dovela de cierre del vano 6. 


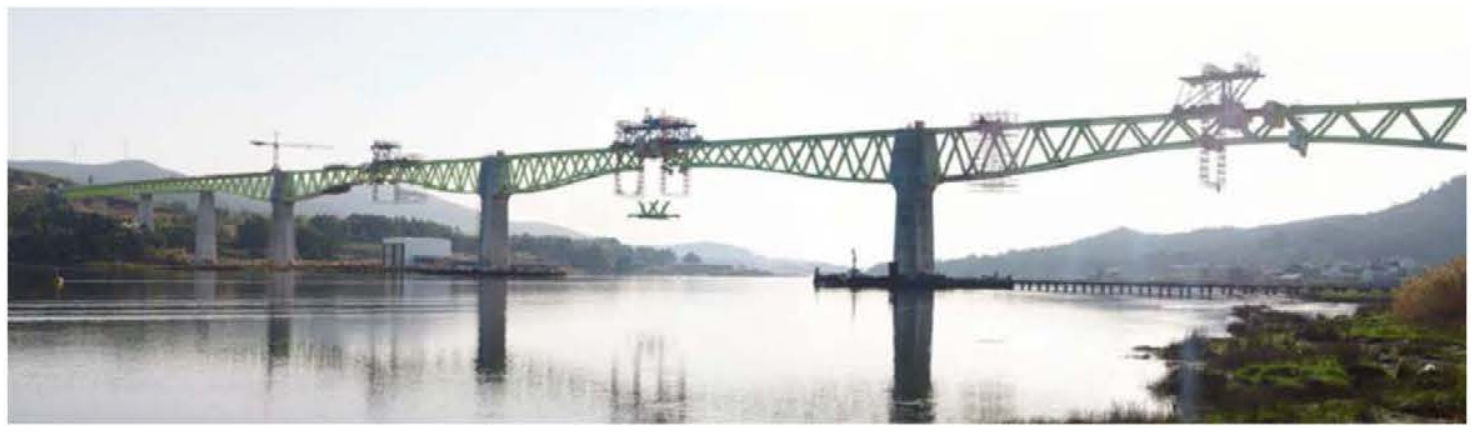

Figura 44. Izado de dovela de cierre del vano 7.

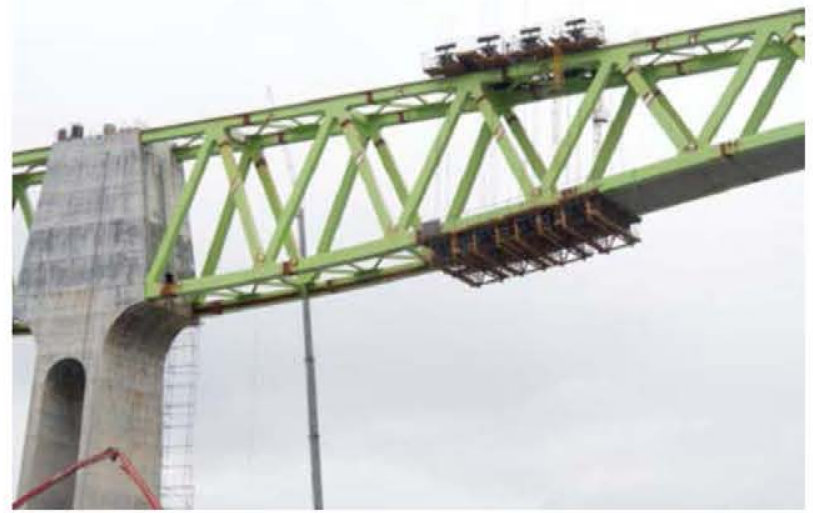

Figura 45. Vista del carro de encofrado del hormigón de fondo.
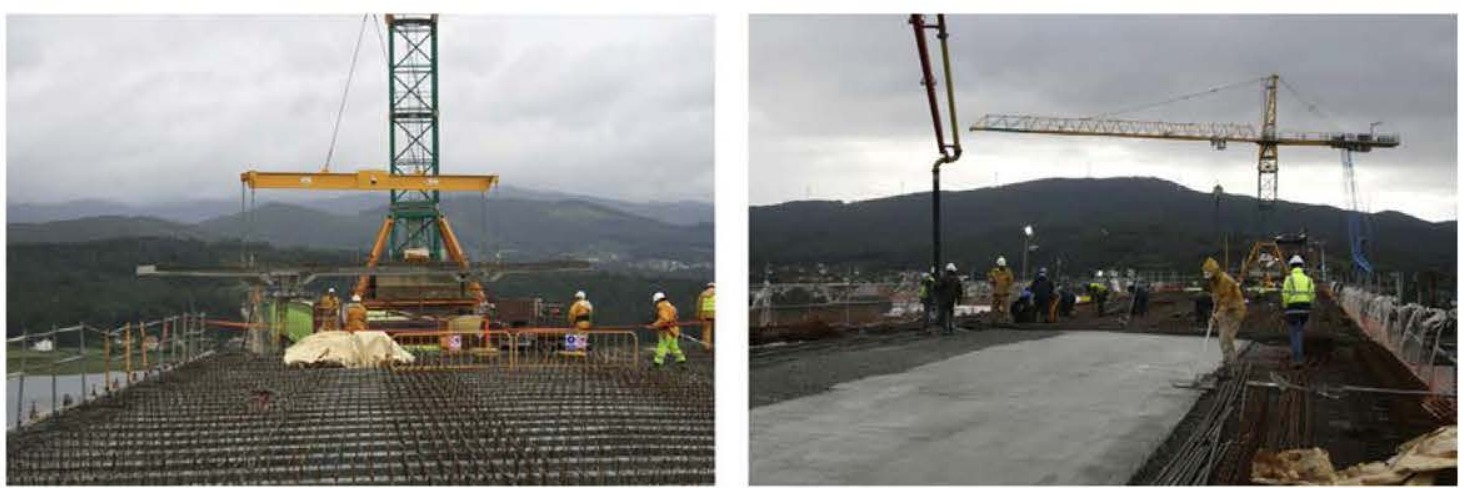

Figura 46. Colocación de prelosas de todo el ancho en el tablero y hormigonado de losa superior.

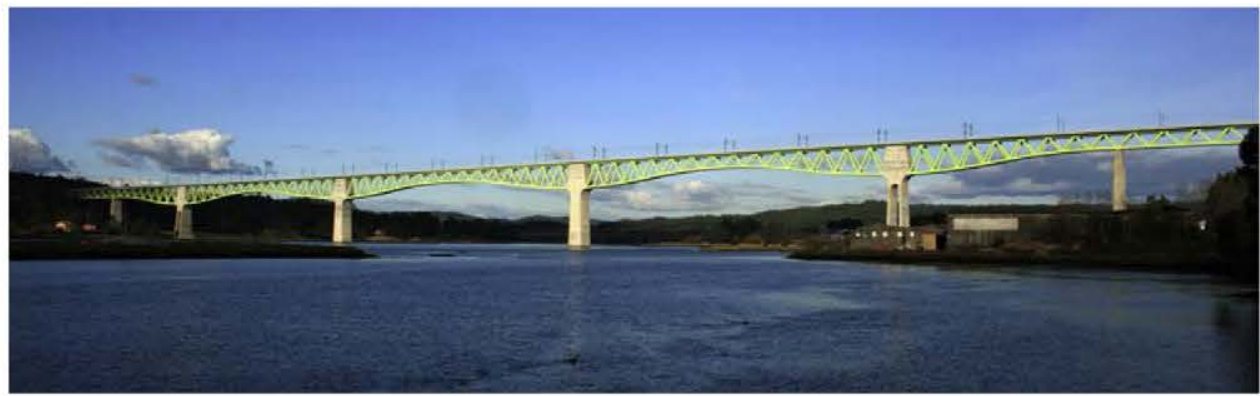

Figura 47. Vista del viaducto concluido. 


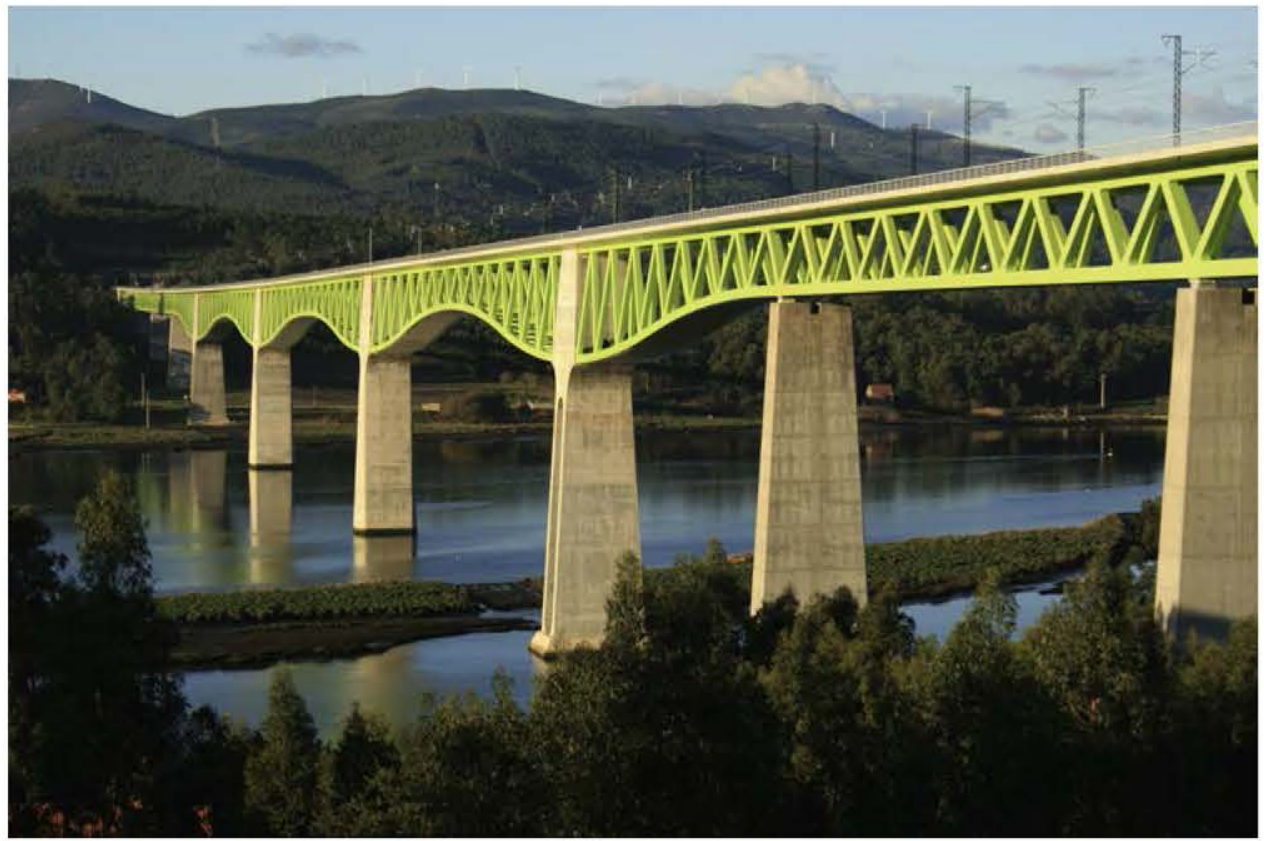

Figura 48. Vista lateral del viaducto concluido.

\section{Conclusiones}

Con la puesta en servicio del Eje Atlántico de Alta Velocidad entre Pontevedra y A Coruña, en la primavera de 2015, el viaducto sobre el río Ulla (figs. 47 y 48 ) se ha convertido en el récord del mundo en la tipología de celosía mixta de alta velocidad, con 3 vanos de $225+240+225 \mathrm{~m}$ que superan al del puente de Nantenbach, con $208 \mathrm{~m}$ de luz, que ostentaba el récord desde su conclusión en 1993.

Como se ha descrito en el artículo, hay un buen número de circunstancias que hacen del viaducto del río Ulla un viaducto único, entre las que cabe citar:

- El empleo de tres complejísimos procesos constructivos diferentes: con izado de vanos completos, lanzamiento de vanos laterales y avance por voladizos sucesivos de dovelas metálicas en los vanos principales.

- El empleo de medios auxiliares únicos fabricados ex profeso para la ejecución del viaducto.

- El empleo de recintos de tablestacados de grandes dimensiones (hasta $68 \mathrm{~m}$ ) para la ejecución de las cimentaciones de las pilas de la ría en seco.

- La fabricación tumbada de la dovela cero de las pilas centrales del cauce (de $35 \mathrm{~m}$ de longitud y $17,5 \mathrm{~m}$ de altura y $2 \times 375 \mathrm{t}$ de peso) y su compleja operación de verticalizado e izado, que ha supuesto un reto nunca realizado hasta la fecha.

- El empleo de aceros de alta resistencia termomecánicos poco habituales en España en puentes (S-460-M y S-460-ML).

- La instalación de tres grandes naves de montaje de dovelas en obra con gálibo libre interior de $20 \mathrm{~m}$.

- El transporte e izado de dovelas dentro de la obra de hasta $17,5 \mathrm{~m}$ de altura y $300 \mathrm{t}$ de peso con carros con multiejes autopropulsados.
- El empleo de hormigones con alta resistencia en las pilas centrales (HA-70) y hormigones autocompactables.

- La realización de hormigonados continuos sin interrupción en cimentaciones con más de $5.000 \mathrm{~m}^{3}$ durante más de $70 \mathrm{~h}$ con control de la temperatura del fraguado con termopares.

- Un sistema de control de calidad de la ejecución de la estructura metálica pionero en España que marcará la línea del control en puentes y viaductos metálicos y mixtos.

Todas estas singularidades han hecho merecedor al viaducto del río Ulla del premio Construmat 2015 en la modalidad de mejor Obra Civil, del premio San Telmo 2015 concedido por la demarcación de Galicia del Colegio de ingenieros de Caminos, Canales y Puertos, y del accésit al VII premio Acueducto de Segovia 2015 concedido por el Colegio de Caminos Canales y Puertos y la Fundación Caminos.

\section{Anexo 1. Principales participantes en el proyecto y la obra}

\footnotetext{
Proyecto del Viaducto del río Ulla

Promotor:

Dirección General de Ferrocarriles, Ministerio de Fomento

Director del Proyecto: Ángel Lanzarote

Concepción y proyecto del Viaducto del río Ulla:

IDEAM: Francisco Millanes, Miguel Ortega, Luis Matute, Carlos

Gordo, Jesús Martín, Alicia de Frutos

Asistencia Técnica para la redacción del proyecto:

UTE IDEAM-Euroestudios

Autor del Proyecto: Francisco Millanes (IDEAM)

Construcción del Viaducto del río Ulla

Promotor y Dirección de Obra:

ADIF Alta Velocidad / Dirección General de Ferrocarriles, Ministerio de Fomento
} 
Dirección de Obra: Rubén A. Estévez (ADIF AV) / Marina Soler (Ineco)

Asistencia Técnica a la Dirección de Obra:

TYPSA: Luis Conde, Carmen Casas, Mar Cariño, Jaime Losada Asesoría especializada en estructuras a la Dirección de Obra y a la Asistencia Técnica a la Dirección de Obra:

IDEAM: Francisco Millanes, Miguel Ortega, Pedro Atanasio, Adrián Sánchez, Juan Luis Mansilla, Jokin Ugarte, Carlos Gordo

Entidad de Control de la estructura metálica de la Dirección de Obra: Applus

Control de calidad del resto de la obra (por parte de la Dirección de Obra):

Enmacosa

Constructora:

UTE río Ulla. Dragados-TECSA

Gerente: Pedro Chico / Jorge Sánchez; Jefe de obra: Imanol Güemes; Coordinador de ejecución de la estructura metálica: David Mouriño; Jefa de Calidad: Susana Cuevas

Apoyo Técnico a la Constructora:

Servicios Técnicos de Dragados: Luis Peset, Felipe Tarquis, Susana

López Manzano, Pilar Hue

Fhecor ingenieros consultores: Hugo Corres, Julio Sánchez, Javier de Cabo, Miriam Alonso

Talleres Metálicos:

Ascamón-Joama / Martifer / Emesa / Dizmar

Entidad de Control de la estructura metálica del Contratista:

Alfainstant y AMT

Aparatos de apoyo esféricos:

VSL

Lanzamiento de vanos 3 y 4, e izado de dovelas de vanos 5, 6, 7, 8 y 9 de canto variable:

VSL

Izado de vanos 10, 11 y 12 y transporte de dovelas en la obra:

ALE

Subcontratistas de cimentaciones:

Cimentalia, Geocisa, Rodio-Kronsa, Pilotes Posada, Amilibia

Subcontratista fabricante de prelosas:

Precon

\section{Bibliografía}

[1] F. Millanes, L. Matute, M. Ortega, D. Martinez, E. Bordó, Desarrollo de soluciones mixtas y metálicas para viaductos de las líneas de alta velocidad españolas, Hormigón y Acero 62 (259) (2011) 7-27.

[2] O. Schwarz, W. Zellner, R. Saul, Three bridges with the longest spans for the new high-speed railway lines, SEI 2 (2) (1992) 87-92, IABSE.

[3] F. Millanes, L. Matute, M. Ortega, C. Gordo, «El viaducto sobre el río Ulla en el Eje Atlántico de Alta Velocidad: una celosía mixta de $240 \mathrm{~m}$ de luz», V Congreso ACHE, Barcelona, 2011.

[4] S. López, I. Muñoz, F. Tarquis, F. Millanes, M. Ortega, R. Estévez, et al., «Ejecución de las cimentaciones del viaducto del río Ulla en la ría de Arosa», V Congreso ACHE, Barcelona, 2011.

[5] R.A. Estévez, F. Millanes, M. Ortega, A. Ferrandis, «Nuevos planteamientos en el control de la ejecución de puentes metálicos: el viaducto mixto sobre el río Ulla», V Congreso ACHE, Barcelona, 2011.

[6] F. Millanes, M. Ortega, P. Atanasio, J.L. Mansilla, D. Mouriño, «Concepción de los detalles de la estructura metálica del viaducto del río Ulla», VI Congreso ACHE, Madrid, 2014.

[7] AENOR AEN/CTN 140 (2008): Eurocódigo 3 «Proyecto de estructuras de acero». Parte 1-9: «Fatiga». UNE-EN 1993-1-9.

[8] F. Millanes, J. Pascual, M. Ortega, Viaducto 'Arroyo las Piedras'. Primer viaducto mixto de las Lineas de Alta Velocidad Españolas, Hormigón y Acero (243) (2007) 5-38.

[9] F. Millanes, E. Bordó, J. Martín, J.L. Mansilla, Viaducto de Archidona (L.A.V. Antequera-Granada), $3.150 \mathrm{~m}$ de dintel mixto sin juntas de dilatación, Hormigón y Acero 270 (2013) 7-32.

[10] S. López, F. Tarquis, D. Mouriño, J. Sanchez, R.A. Estévez, M. Ortega, «Ejecución de los vanos de acceso del viaducto del río Ulla de la margen izquierda mediante lanzamiento», VI Congreso ACHE, Madrid, 2014.

[11] D. Mouriño, S. López, J. Sanchez, F. Tarquis, R.A. Estévez, M. Ortega, «Izado de los vanos de acceso de la margen derecha del viaducto del río Ulla», VI Congreso ACHE, Madrid, 2014.

[12] M. Ortega, J.A. Becerra, R.A. Estévez, P. Chico, H. Corres, J. Sanchez, «Ejecución de los vanos de canto variable por avance en voladizos sucesivos del Viaducto del río Ulla», VI Congreso ACHE, Madrid, 2014. 


\title{
Viaduct over Ulla River in the Atlantic high speed railway line: A composite (steel-concrete) truss world record
}

\author{
El viaducto del río Ulla en el Eje Atlántico de Alta Velocidad: una celosía mixta récord del mundo \\ Francisco Millanes Mato , Miguel Ortega Cornejo ，Rubén A. Estévez Sánchez
}

\begin{abstract}
With the commissioning of the Atlantic high speed railway line between Pontevedra and A Coruña, in the spring of 2015, the viaduct over the river Ulla has achieved the world record in the typology of high speed composite truss bridge, with three main spans of $225+240+225 \mathrm{~m}$. This exceeds the Nantenbach Bridge over Main River in Germany, which held the record since its conclusion in 1993 with a $208 \mathrm{~m}$ span.

This article describes in detail the design and the main features of the viaduct, the new system of quality control of the execution implemented with great success. A description is also presented on the complex and outstanding construction processes used for the execution of the deck of the bridge and the foundation of the piers located on the river, with the exceptional temporary works that have been fabricated "ex profeso" for the execution.
\end{abstract}

Keywords: Steel structure; Truss; Double composite action; Balanced cantilevers; Lifting; Launching

\section{Resumen}

Con la puesta en servicio del Eje Atlántico de Alta Velocidad entre Pontevedra y A Coruña, en la primavera de 2015, el viaducto sobre el río Ulla se ha convertido en el récord del mundo en la tipología de celosía mixta de alta velocidad con tres vanos de $225+240+225 \mathrm{~m}$ que superan al del puente de Nantenbach sobre el río Main, en Alemania, que ostentaba el récord desde su conclusión en 1993 con 208 m de luz.

Este artículo describe con detalle la concepción y las principales particularidades del viaducto, el novedoso sistema de control de calidad de la ejecución implementado con gran éxito, así como los complejos y singulares procesos constructivos empleados para la ejecución del tablero y de las cimentaciones de las pilas en el cauce, junto con los excepcionales medios auxiliares que ha sido necesario fabricar ex profeso para su ejecución.

Palabras clave: Estructura metálica; Celosía; Doble acción mixta; Avance en voladizos; Izados; Lanzamiento 


\section{Introduction}

The viaduct over River Ulla at the mouth of Arosa Estuary constitutes the most prominent structure on the Atlantic high speed railway line between Pontevedra and A Coruña in northwestern Spain. Its three centre spans are now the world's longest on a composite truss bridge for high-speed rail traffic [1].

Its site near the Arosa Estuary, in extraordinarily beautiful surroundings subject to strict environmental protection, induced the Directorate General for Railways to shortlist an ideas competition. The solution submitted by IDEAM was awarded the contract for the detailed design, which was geared to meeting the following objectives:

- to sustain the aesthetic proposed and ensure that the structure would blend into its surrounds;

- to minimise the number of piers in the riverbed while building the viaduct to the technical requirements of a high speed railway bridge at an affordable cost;

- to deploy construction processes with the lowest possible impact on the riverbed and banks;

- to maximise transparency and minimise the visual impact on the surrounding landscape.

The prominence of the works and their inevitable effect on the landscape called for a design that would blend suitably with or even enhance the surrounds. The solution, inspired by the beauty and environmental variety of the estuary, was based on a highly transparent truss design in which depth transitions between the riverbed piers were smoothed with fish belly-shaped curvatures. The resulting deck appears to 'float' over the estuary (Figs. 1 and 2), in a deliberate digression from the traditional angular variations in depth that would have rendered the support afforded by the centre piers overly conspicuous.

With its pearl grey concrete and light green steel truss, the viaduct blends discreetly and elegantly with the surrounds, where the predominantly green woodlands and grey river are sprinkled with bright reflections on the occasional sunny day and filtered through a cloak of mist and fog on so many others.

\section{Structural concept and description}

The conditioning factors described in the Introduction informed a solution consisting in a continuous truss bridge with a steel and concrete structure that would afford double composite action in the negative moment areas. The typical approach spans were to measure $120 \mathrm{~m}$ and the three long main spans 225 , 240 and $225 \mathrm{~m}$, the longest $20 \%$ longer than the former world record 208-m centre span on Nantenbach Bridge over the River Main [2] in Germany.

The design for the $1620 \mathrm{~m}$ viaduct envisaged 12 spans $(50+80+3 \times 120+225+240+225+3 \times 120+80$ m) (Fig. 1) and two joints fitted with track expansion devices at the abutments.

The five variable depth spans (Fig. 2) were designed to measure $17.90 \mathrm{~m}$ over the bearings $(17.50 \mathrm{~m}$ steel) and $9.15 \mathrm{~m}$ (8.75 $\mathrm{m}$ steel) at mid-span, and the likewise composite truss on

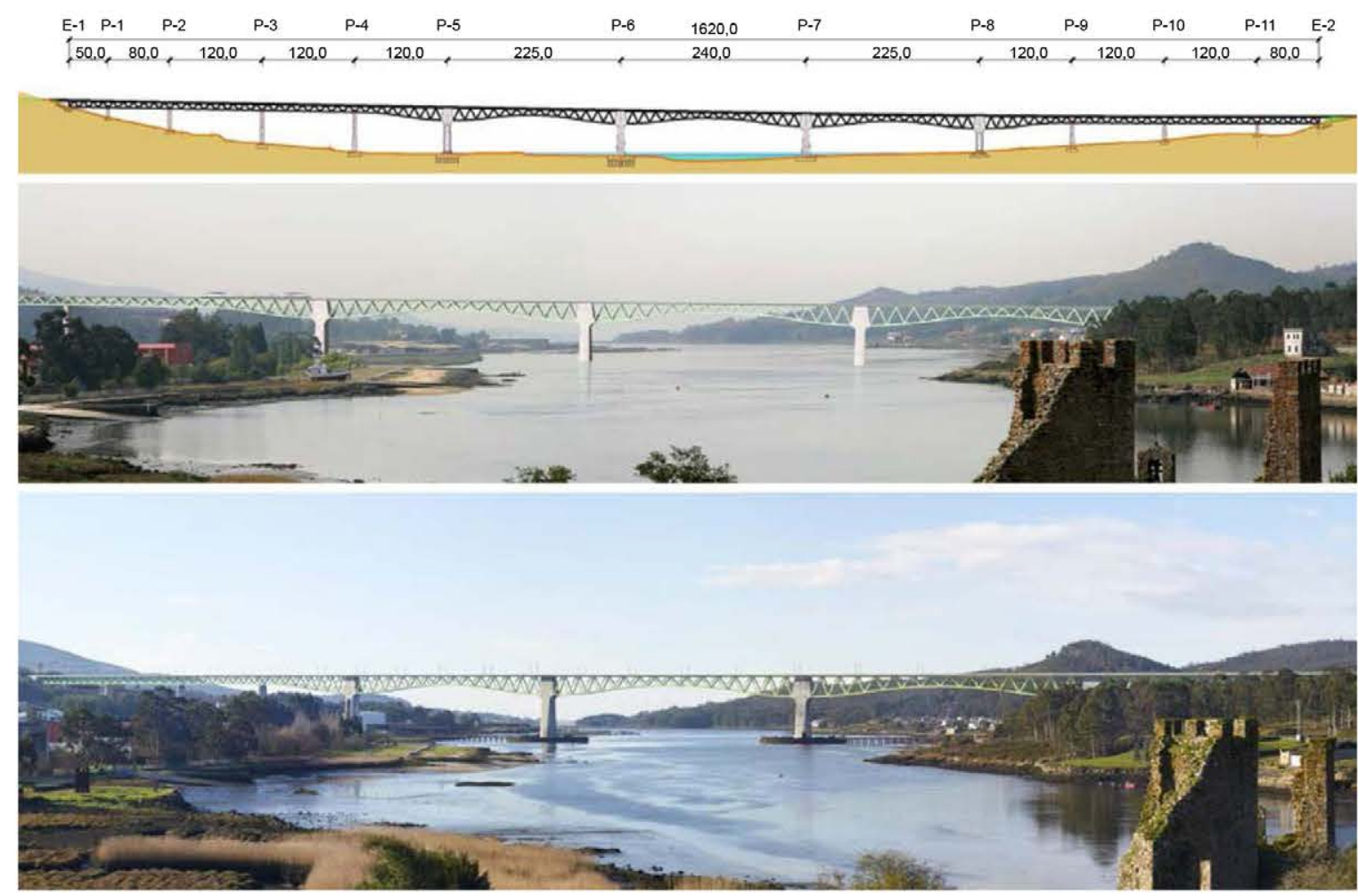

Figure 1. Elevation drawing, simulation and photograph of the finished viaduct over the River Ulla. 


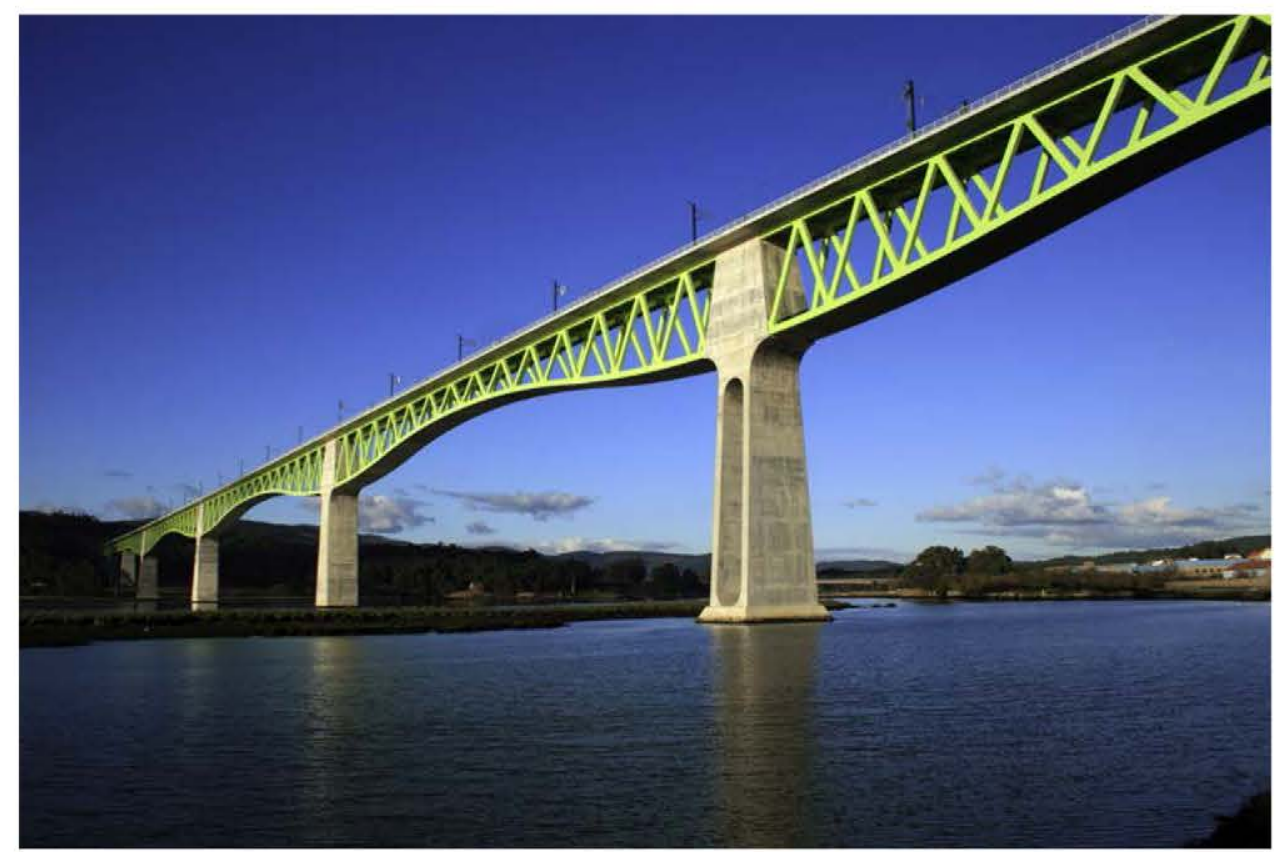

Figure 2. Variable depth centre spans. Pier 5 in the foreground.

the 120-m approach spans were to have a constant total depth of $9.15 \mathrm{~m}$.

The four artfully designed goblet-shaped centre piers (P5 to P8) were to be stiffly secured to the composite truss deck, forming a composite portal frame sufficiently stiff in the three centre $(225+240+225-\mathrm{m})$ spans (Fig. 2) to bear the strain induced by high speed and the concomitant live loads on alternating spans.

The design optimised the stiffness in the four main piers to constrain deck rotation as required, while controlling the bending moment transferred via frame action to the pier foundations to elude any need for over-dimensioning [3].

To that end, the main side piers (P5 and P8, Fig. 2), located on the outer ends of the 225-m spans, were designed with two unengaged walls restrained at the foundations and pierhead. This arrangement would control the moment restraint deriving from the hugely different lengths of the two adjacent spans $(225$ and $120 \mathrm{~m})$, as well as the bending moment stemming from temperature- and rheology-induced pierhead displacement. These effects would be perceptibly greater in P5 and P8 than in the two centre piers (P6 and P7) due to the substantially longer distance, on the order of $350 \mathrm{~m}$, to the zero displacement point on the deck.

The remaining approach piers were designed to have conventional hollow rectangular sections and variable length- and transverse dimensions. In the design, the spherical deck bearings on these piers were longitudinally detached, with transverse displacement constrained in one of them and free on the other.

The Warren truss envisaged in the design would consist in 15-m long modules in which the two lateral sets of nodes on the upper chord would be spaced $6 \mathrm{~m}$ apart and the diagonal web members slanted at around $45^{\circ}$ from the horizontal in the constant depth area; the sheet steel would form parallelograms $0.80 \mathrm{~m}$ wide and $1.00 \mathrm{~m}$ deep on the upper chords and diagonals and $0.80 \mathrm{~m}$ wide and $1.20 \mathrm{~m}$ deep in the bottom chord.
In the design, the upper chords were crowned with an additional closed profile welded to the upper flange to be embedded in the concrete slab. By welding the connectors to the sides of this member, the load transfer axis in the connection would be closer to the barycentre of the upper slab, thereby reducing eccentricity-induced parasitic bending moments on the connection (Fig. 3).

$\mathrm{S} 355-\mathrm{J} 2+\mathrm{N}$ and S355-K2+N (for sheets over $60 \mathrm{~mm}$ ) quality steel was specified for the truss in the approach spans, and S-460 $\mathrm{M}$ and S-460-ML (for sheets over $65 \mathrm{~mm}$ ) in the three variable depth, longer centre spans.

The top slab depth was to vary from 0.46 to $0.25 \mathrm{~m}$ and the RC-35 cast-in-place concrete was to be poured on composite, full-width precast slabs.

In the design, the RC-50 bottom slab would be cast between and secured to the two bottom chords to establish double composite action in the areas subjected to negative moments. In all other areas, the bottom concrete slab would consist in merely formal, i.e., non-structural, concrete to facilitate inspection and maintenance (Fig. 4). Except as otherwise indicated below, the aforementioned design specifications were implemented in viaduct construction.

\section{Foundation construction}

Further to the geological survey, the uppermost level of the ground under the alignment consists in loose colluvium on the banks and alluvium in the estuary, both resting on a granite substratum with a more or less altered surface.

The abutments and simple land-side approach piers P1 to P4 and P8 to P11 have shallow foundations (footings) built over the granite substratum where the mean allowable stress is $1.00 \mathrm{MPa}$. Two of the three riverbed piers (P5 and $\mathrm{P} 6$ ) rest on 1.5-m diameter capped piles restrained in the sound granite, 


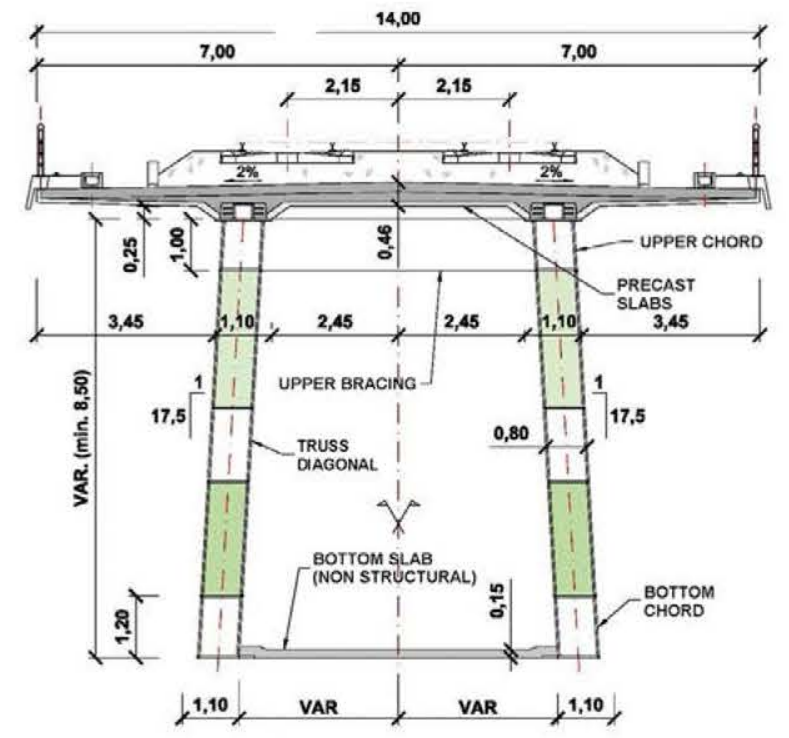

SAGGING CROSS SECTION

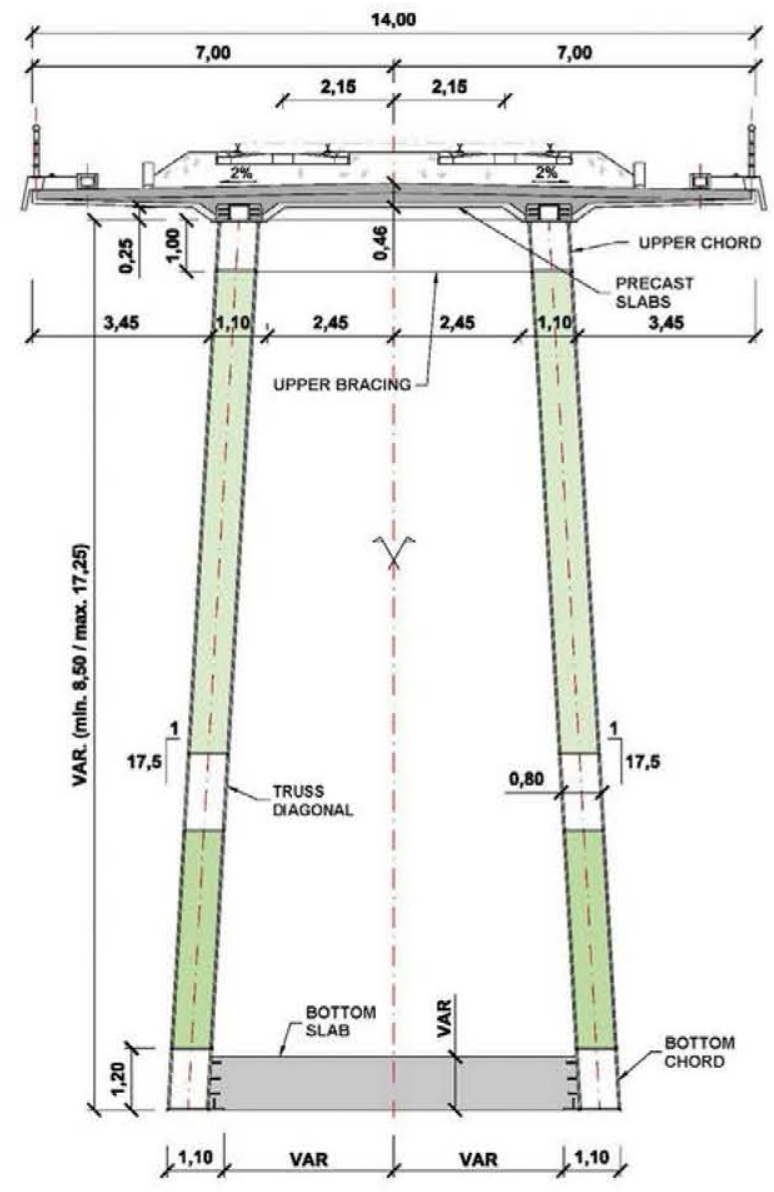

HOGGING CROSS SECTION

Figure 3. Typical cross-sections.

while the third (P7) has shallow foundations built directly on the rocky substratum.

\subsection{Provisional wharf to access riverbed foundations}

A provisional wharf was built to provide access to the foundations for the three riverbed piers (Fig. 5) and carry the steel deck segments to the positions from which they could be hoisted into place. The nearly exclusive use of land-based resources for construction that this entailed hastened works progress while respecting the environmentally protected banks.

The deck over the wharf consisted in a series of statically determinate steel spans $6 \mathrm{~m}$ long and $14 \mathrm{~m}$ wide (Fig. 6) to accommodate two $4.5-\mathrm{m}$ traffic lanes separated by a $1-\mathrm{m}$
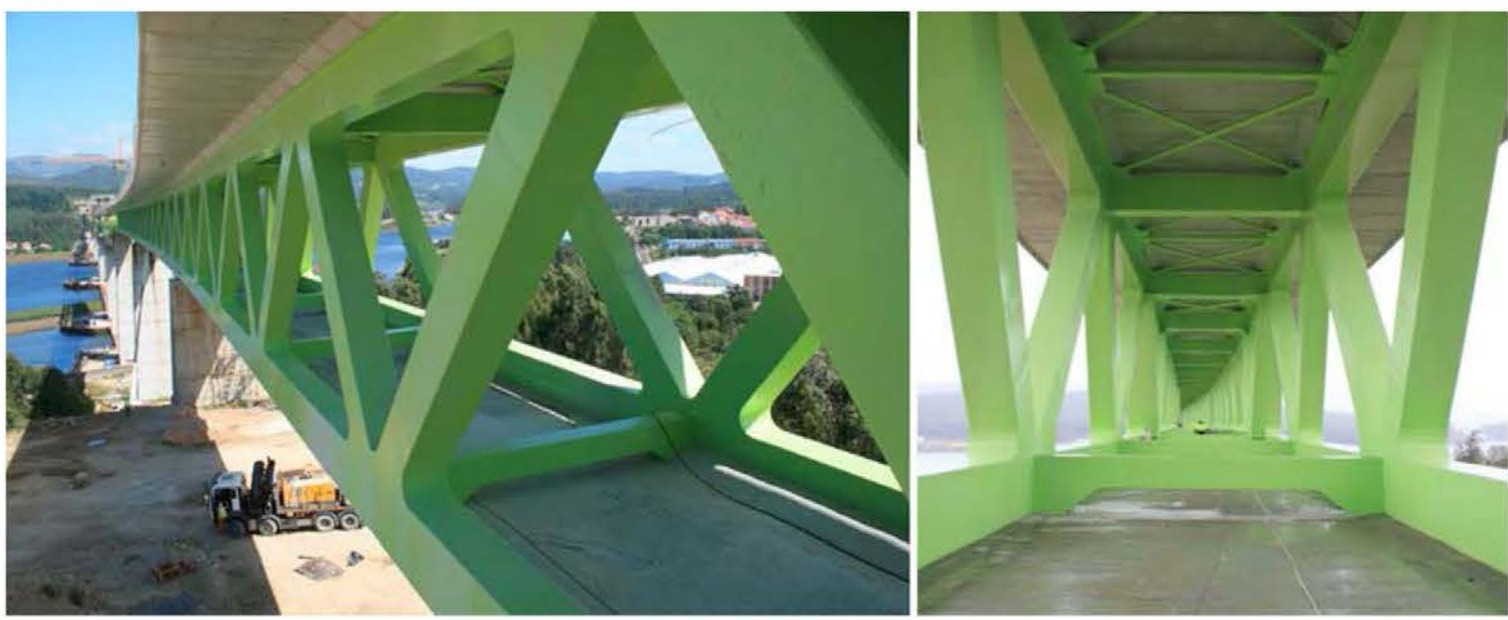

Figure 4. Side view and interior of the truss viewed from the bottom concrete slab. 


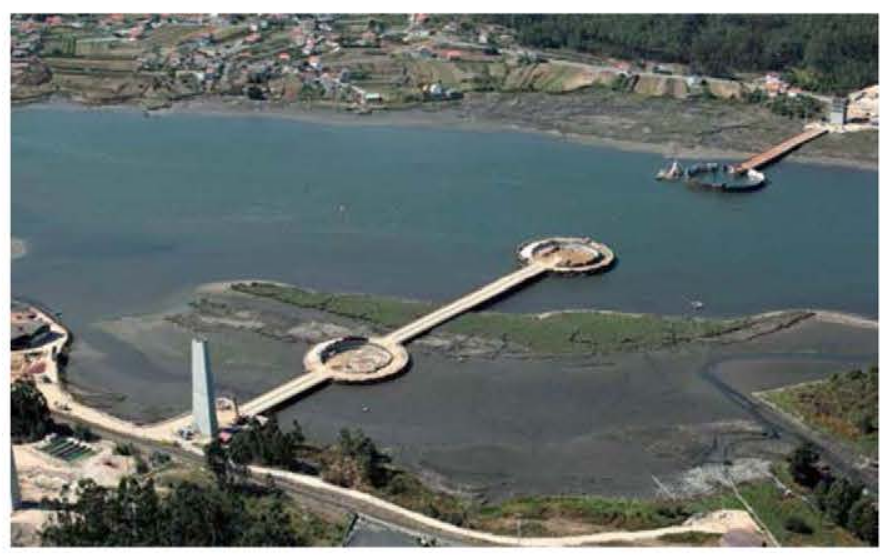

Figure 5. Wharf and foundation enclosures for riverbed piers P5, P6 and P7.

central reserve, plus two 2-m wide outer corridors divided in two, half for laying supply pipes for the construction areas and the other half for pedestrian traffic. This deck was supported by lintels resting on steel CHS piles $800 \mathrm{~mm}$ in diameter and $12 \mathrm{~mm}$ thick, driven with a pile hammer to the sound layer. Ref. [4] contains a detailed description of the typical cross-section and construction process for the wharf.

\subsection{Provisional sheet pile enclosures in riverbed foundations}

Provisional enclosures (Fig. 5) were built around the sites for the three riverbed piers for dry construction of the foundations. These enclosures were also used as working platforms to erect the piers and hoist the steel deck segments into position.

These structures, with 62-, 68- and 65-m outer diameters at piers P5, P6 and P7, respectively, consisted in sheet piles forming two concentric rings spaced at $10 \mathrm{~m}$ and designed to bear bending moments. Both rings were hooped with circular braces positioned at several levels, the steel hoops on the outer ring to bear tensile and the reinforced concrete hoops on the inner ring to bear compressive stress [4].

The inner sheet piles in piers 6 and 7 were driven to rock depth. The excavation depths were $8 \mathrm{~m}$ in P5, $10.5 \mathrm{~m}$ in P6 and $13 \mathrm{~m}$ in P7 (Fig. 7). At peak high tide the estuary rises to $2.54 \mathrm{~m}$ above sea level, very near the elevation of the enclosure platforms $(+3.0 \mathrm{~m})$. Such deep excavations, on the order of $15 \mathrm{~m}$, and the presence of water around the exteriors, called for enclosures with substantial retaining power.

The outer rings were braced with perimetric S-355 steel hoops. Piers 5 and 6 were hooped with one such member each, while two were required in pier 7 (Fig. 7).
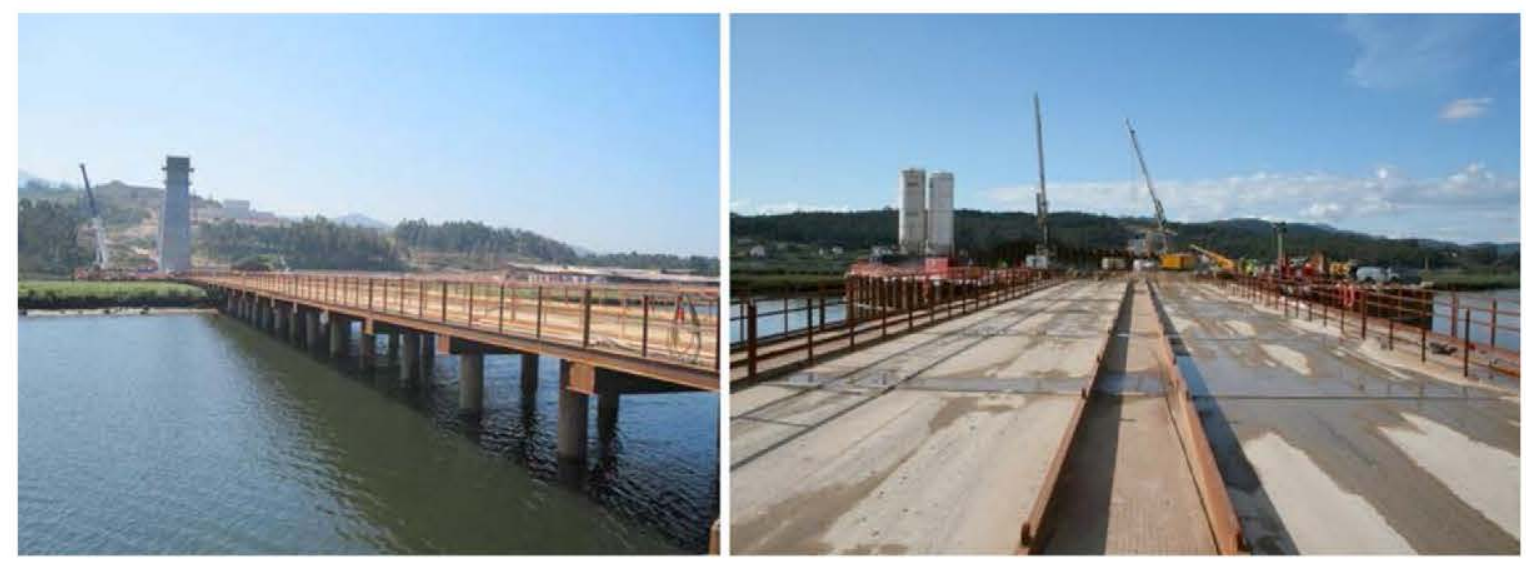

Figure 6. Wharf for accessing pier P5, P6 and P7 foundations.

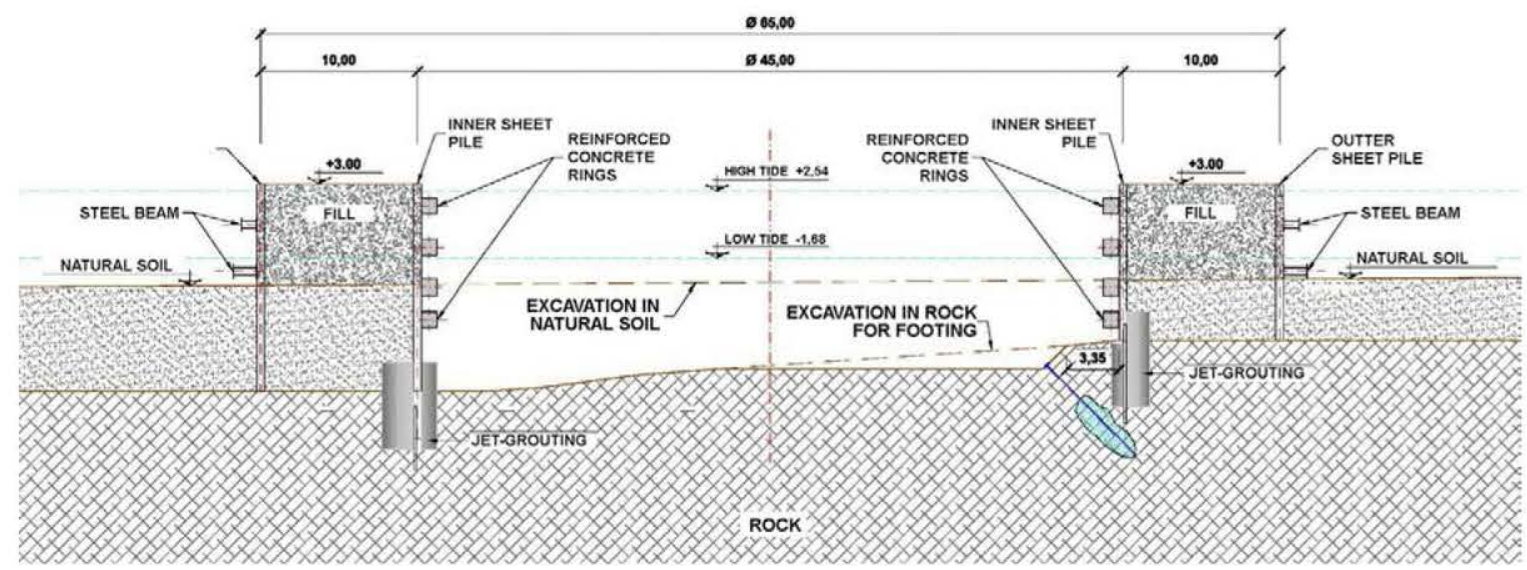

Figure 7. Sheet steel enclosure around pier P7 foundations. 


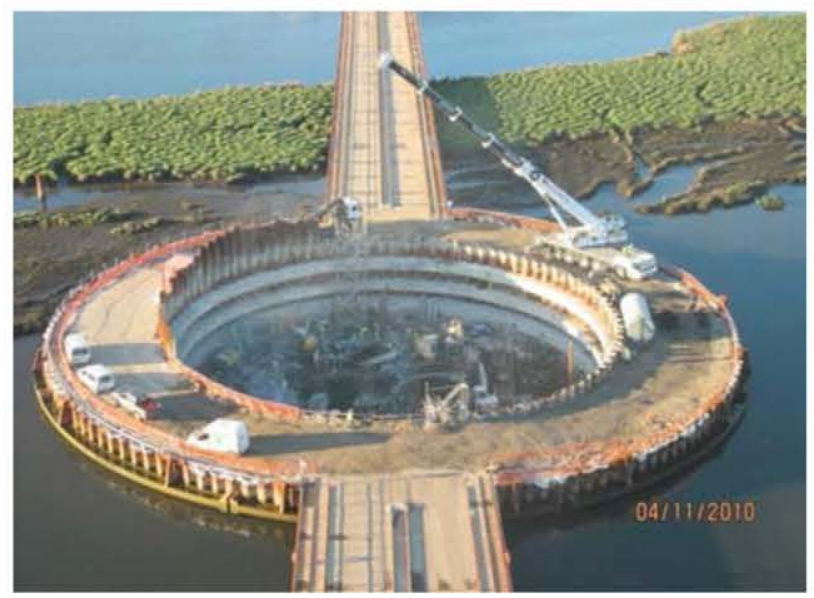

Figure 8. Inner excavation in P5 enclosure.

The compression braces circling the inner sheet pile walls were $\mathrm{RC}-40$ hoops with a $1.0 \times 1.0-\mathrm{m}$ rectangular cross-section. Three braces were needed in piers 5 and 6 (Fig. 8) and four in P7.

In addition, a double ring of columns was jet-grouted around the inner sheet pile wall in all three enclosures to seal the interface between the rock and the sheet piles to prevent leakage. These columns also played a structural role in pier 6 , where the bearing capacity inherent in their circular shape provided lateral support for the toes of the sheet piles.

As greater strength was needed to restrain the toes on the inner sheet piles in pier 7 , which became detached during excavation, a reinforced micropile was built at the foot of each sheet pile to resist the resulting shear stress (Fig. 7).

\subsection{Enclosures and riverbed foundations}

The sheet piles for the outer rings in the enclosures were vibration driven from the wharf and subsequently braced with steel hoops.

The backfill for the inner enclosure ring was dumped from lorries standing on the wharf into the stipulated space, previously lined with geotextiles to minimise the outflow of fines into the estuary.
The sheet piles for the inner enclosures were then driven from the surface of the fill, after which the feet of the sheet piles were superjet-grouted. Lastly, the P7 enclosure was micropiled from the platform.

Pier 5 foundations consisted in 42 capped piles $1.5 \mathrm{~m}$ in diameter, while in pier 6,56 piles were driven $1.5 \mathrm{~m}$ into the grade I-weathered granite substratum. In both cases the operations were performed from the top of the enclosure fill at an elevation of $+3.0 \mathrm{~m}$. The piles were sheathed in re-usable casing until they struck rock; the use of sludge was avoided to prevent possible leakage to the estuary. The casing was driven until contact was made with the more or less altered rocky substratum or, where it appeared, with very dense, compact granite gravel. After all the piles were driven, the enclosures were excavated (Fig. 8), the hoops were built at the respective levels and the pile heads were cut down (Fig. 9) to accommodate the rebar (Fig. 10) and cast the concrete for the pile caps.

The pier 5 and 6 pile caps measure $30 \times 24 \times 4.5 \mathrm{~m}$ (Fig. 10) and $34.5 \times 30.5 \times 5 \mathrm{~m}$, respectively; the bottom pile cap elevation is $-5.0 \mathrm{~m}$ in the former and $-7.5 \mathrm{~m}$ in the latter.

Given these dimensions which translated into a volume in the larger of the two of $5260 \mathrm{~m}^{3}$, concrete casting was hardly an insignificant operation. Concrete was poured continuously, in one case for over $50 \mathrm{~h}$, in the three riverbed pier foundations. Several types of concrete were used, depending on the layer of the pile cap. SCC-35 self-consolidating concrete was applied in the bottom $0.7 \mathrm{~m}$ to ensure adequate placement around the dense bottom reinforcement; conventional vibrated RC-35 was used in the middle area; and FRC-35 fibre-reinforced was poured in the uppermost metre to prevent surface cracking. Furthermore, in the inner area that interfaces with the concrete pier, the material used was of the same quality in both, RC-70. The RC-35 piles were designed for service loads of $8 \mathrm{MPa}$.

The shallow foundations under pier 7 consist in a $27 \times 24 \times 5.25$-m footing, set at a depth of 8.6-m. The lean concrete (RC-15) across which the footing rests on the grade III(or lower) weathered granite substratum bears a maximum cobarycentric stress of $0.76 \mathrm{MPa}$ and a maximum peak stress of $1.00 \mathrm{MPa}$ (Fig. 11).

Upon completion of the riverbed foundations, the enclosures were filled and the inner hoops demolished, in the reverse order of construction during the filling operation. The inner sheet piles

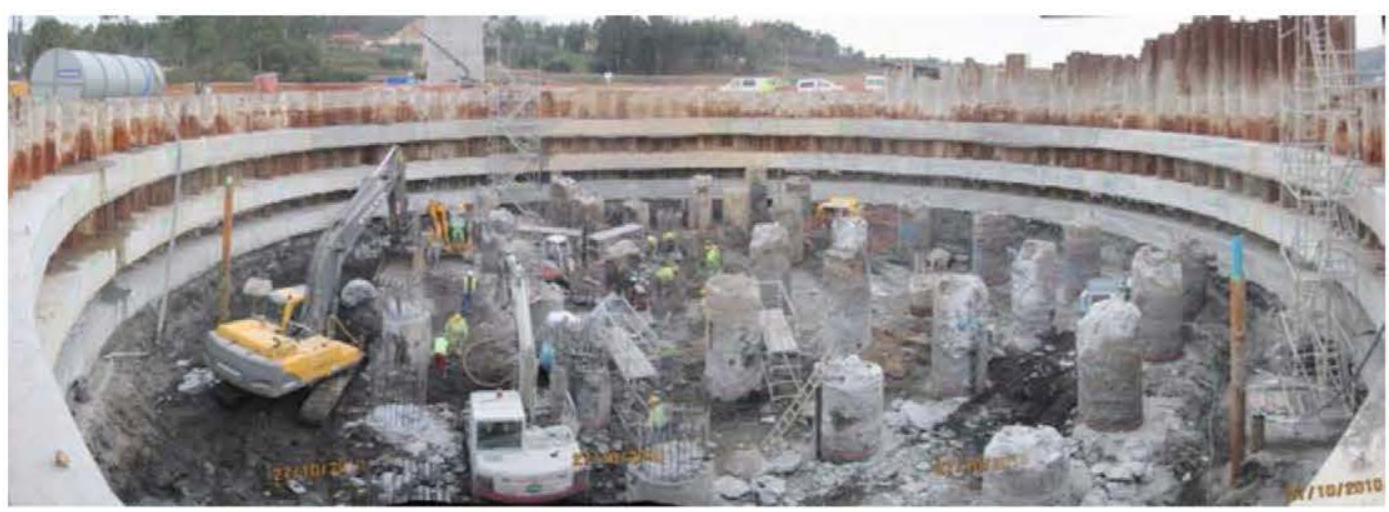

Figure 9. Cutting down P5 foundation pile heads. 


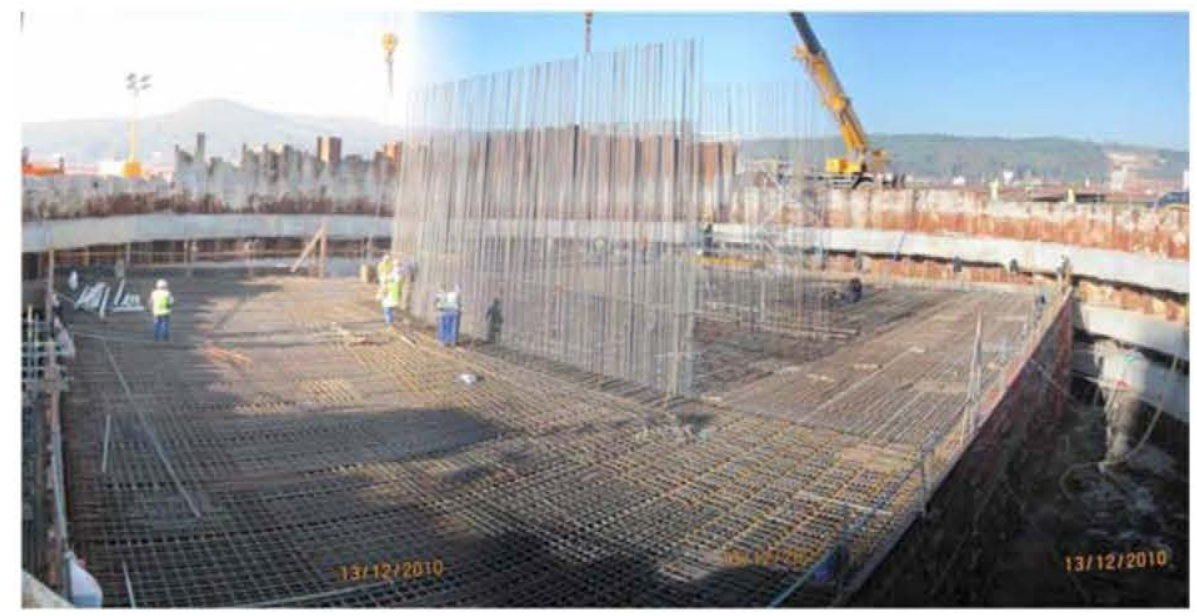

Figure 10. Reinforcement for P5 pile cap $(30 \mathrm{~m} \times 24.5 \mathrm{~m} \times 4.5 \mathrm{~m})$.

were subsequently removed, leaving the islands ready for deck segment transport and hoisting.

The tops of the P5, P6, and P7 pile caps are located on the order of $1 \mathrm{~m}$ below the natural riverbed. Consequently, with the removal of the inner fill, the outer enclosures and the wharf after the deck was erected, the estuary was restored to its original state.

\section{Deck manufacture}

In light of the singularity and complexity of the steel structure and the huge volume (nearly $20000 \mathrm{t}$ ) of structural steel required for the deck, the contractor proposed and the site management agreed to divide manufacture between two groups of steelworks. Initially, half of the viaduct was to be manufactured at the Ascamón-Joama plants in the Spanish region of Asturias and the other half at Martifer, a plant in Portugal. The complexity of the works and the construction deadlines subsequently necessitated the enlistment of two additional manufacturers in the Spanish region of Galicia: Emesa and Dizmar (Fig. 12).

To simplify handling, manufacture and shipping, the trusses forming the deck were conceived from the design phase to

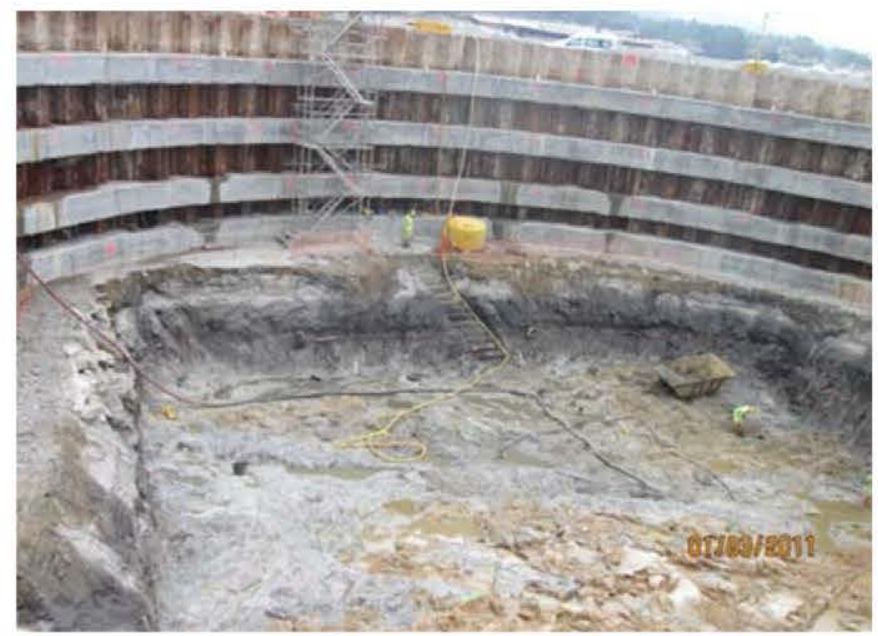

Figure 11. Rocky substratum under P7 prior to pouring the blinding. comprise the following standard components (Fig. 13): upper nodes, upper chords, diagonal web members, bottom nodes, bottom chords, tie girders and cross-braces.

Once all the separate components of each segment were made, they were welded into larger sub-assemblies: node + chord or node + chord + node. These sub-assemblies and the remaining standard components (diagonal web members, tie girders and braces) were shipped to the site yards for final assembly.

To that end, three large-scale segment assembly bays (Fig. 14) were built on site, all with capacity to assemble segments up to $17.5 \mathrm{~m}$ high. In addition, wide spaces were cleared near abutment 1 (A1) and underneath the viaduct between pier P9 and abutment 2 (A2) for use as on-site storage and assembly yards.

\subsection{Steel structure quality control}

The detailed specifications for the project laid down the general criteria on which on-site workmanship quality control for the steel structure was based [5]. The core principle consisted in vesting the contractor with responsibility for accrediting steel

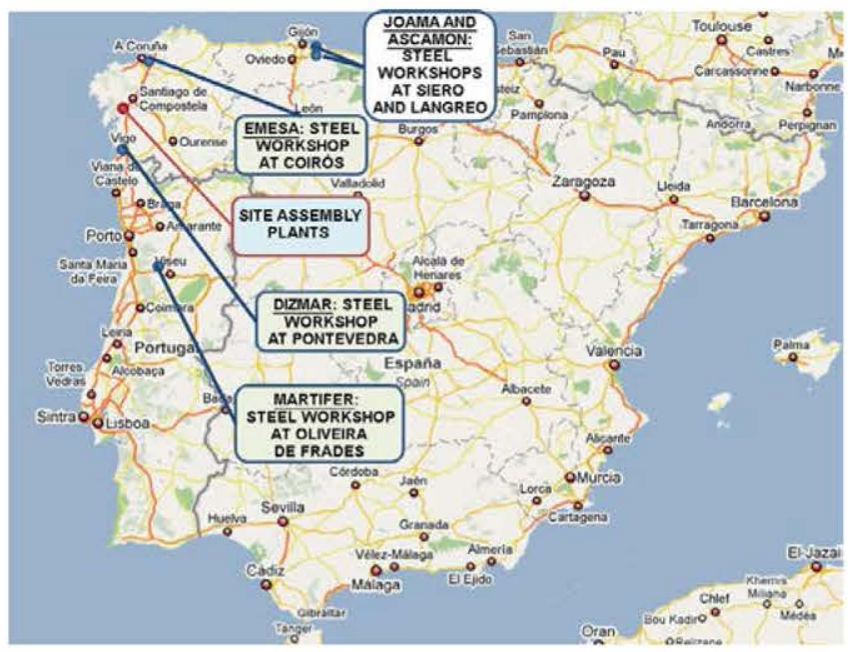

Figure 12. Site map of steelworks commissioned to manufacture the steel struc ture for the viaduct. 


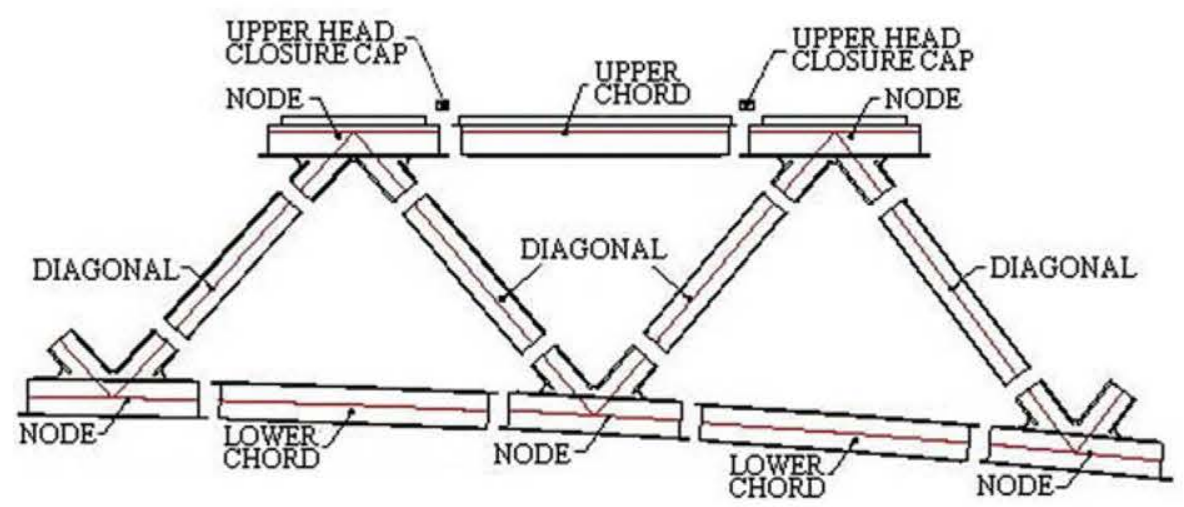

Figure 13. Breakdown of trusses into components manufactured at the steelworks.

structure conformity to the existing legislation on steel structures as part of its quality control programme. The aforementioned specifications stipulated that the contractor could be commissioned to directly conduct and certify all the controls set out therein or assume responsibility for the control conducted by the steelworks, subject to verifying at least $20 \%$ of the plant trials. Consequently, the unit price included the cost of all control tasks incumbent upon the contractor.

The detailed specifications required the contractor to ensure compliance by each and every manufacturer with absolutely all the stipulations on control and hire an independent control body to be permanently on site at the plants and the worksite. This body was responsible for certifying conformity of steel structure workmanship on behalf of the respective manufacturer and reporting to the site management accordingly.

It also supervised and validated at-plant and on-site steel structure manufacture throughout, including material orders and acceptance, approval of welds and weld procedure qualifications and review of the workmanship controls and non-destructive testing (NDT) conduced at the plant.

In addition to continuous plant and quality control system supervision and auditing, the contractor's quality control bodies (Alfainstant and subsequently AMT) verified at least $20 \%$ of the trials conducted by the steelworks. Such verification was not confined to merely repeating non-destructive tests (penetrant testing, magnetic particles inspection, ultrasonic testing and radiographic testing), but during the assembly stage also included inspection of sheet cutting and edging (groove preparation), dimensional controls, pre-weld visual controls and geometric controls and trial assembly, to name a few.

In addition to the contractor's control system, the site management was assisted by a team of engineers (TYPSA), on which the designer firm (IDEAM) sat as expert steel structure adviser, as well as by a further independent quality control body (Applus). The latter audited the contractor's entire workmanship control system and had further verification trials (around 20\%) conducted by a QC laboratory which also performed random NDT verifications.

The contractor's entire control system was set out in three inspection point programmes: steelworks manufacture, worksite plant assembly and on-site assembly, which established the percentage of self-control trials conducted by the manufacturer and the verification trials conducted by the contractor's control body.

The contractor's quality assurance plan described the control systems and mechanisms and contained an organisational chart showing the tasks and responsibilities of all the agents involved in manufacturing and quality control. It also established the mechanisms for reporting incidents and non-conformities.

A manufacturing or assembly incident was defined as a flaw or non-conformity detected at the steelworks during any of the manufacturing stages. All incidents, regardless of their
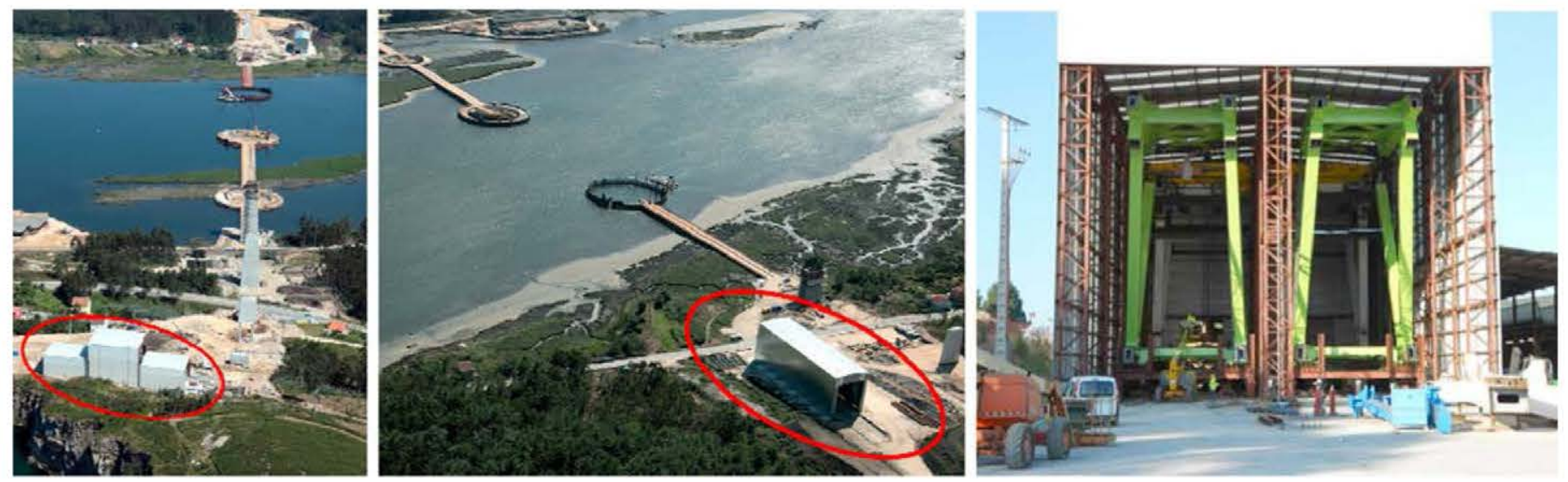

Figure 14. On-site segment assembly bays. 
significance, were reported to the contractor's and the site management's control bodies. Minor incidents were reported by the manufacturer, documented and solved, with no need to suspend production. Incidents of greater significance were reported to the control bodies and the solution agreed upon by the contractor's control body and the manufacturer, based on the repair procedure proposed by the latter.

Workmanship flaws or defects detected by the contractor's control body but not by the manufacturer's quality control system constituted non-conformities.

Non-conformities were filed, for instance, when the steelworks plant detected recurrent incidents in a given process, such as dimensional flaws or defects in typical welds identified by ultrasonic testing, denoting a systematic flaw of much greater significance than an occasional individual flaw or defect calling for process or control procedure restructuring rather than mere sporadic repair.

Non-conformities identified by the contractor necessitated suspension, documentation of the non-conformity, analysis of the cause and proposal not only of an ad hoc solution, but corrective measures to prevent systematic or recurrent defects. Implementation of repairs and resumption of production was contingent upon the site management's validation of the proposal.

The inspection point programmes stipulated specific control procedures and acceptance criteria. Measures were taken prior to works commencement to ensure that all the actors involved in workmanship control were fully aware of the criteria to be applied and the schedules that would be used to validate each specific trial. At the River Ulla Viaduct, control was based primarily on European standards (EN series) and their translation into Spanish (UNE-EN series). Other legislations (essentially American, AWS series and similar) were resorted to only in the absence of relevant EN standards.

After welding, plant sub-assemblies were only authorised for shipping or worksite yard steel segments for on-site assembly where conformity with all the workmanship controls and NDTs laid down in the respective inspection point programme was documented and fully certified.

\subsection{Shop drawings for steel structures}

The complexity of the works, the geographic scatter of the steelworks and the many control agents involved led the contractor to hire a Steel Structure Construction Coordinator, who served as a liaison between the site management and its technical assistance team for steel structure-related matters. One of the foremost responsibilities of the position was to coordinate and unify all technical proposals for adapting, modifying or adjusting a given detail among all the plants.

Another essential factor on which control was based was the system for approving and validating shop drawings for the steel structure.

The steelworks developed detailed shop drawings and cutting sheets for each steel plate from design drawings. Responsibility for accrediting shop drawing and cutting sheets conformity was vested in the contractor, who in turn outsourced the task to a specialised consultant. After the shop drawings were approved, they were forwarded to the site management's technical assistance team for in-plant supervision and authorisation or observations for corrections.

The complexity of the steel structure called for considerable prior study and development of a series of recurrent details by all the parties concerned (steelworks plants, contractor and technical support team, together with the design engineer who assisted the site management) to ensure that the shop drawings contained the solutions for all connections, welds, transitions and specific details to prevent problems from arising during construction. The level of definition of the shop drawings for the steel structure is described in Ref. [6].

Many possible worksite problems were avoided by not accepting under any circumstances drawings for dimensionless or tabulated typical details that failed to represent plate thickness, geometry, angle of incidence, root openings, root faces or groove preparations at all the specific welded connections.

The 131 design drawings defining steel structure details spawned over 6000 shop drawings and nearly 20000 cutting sheets, with precise definitions for each steel plate, connection and weld on the bridge. Such meticulous engineering was imperative to guarantee the satisfactory visualisation of all the details given the need to comply with very strict fatigue resistance standards in light of the mission and nature of this composite high speed viaduct.

\subsection{Assembly sequence drawings}

In addition to the shop drawings and cutting sheets, assembly drawings were prepared for each typical component (upper nodes and chords, diagonal web members, bottom nodes and chords, upper and bottom tie beams and cross-braces), defining:

- the assembly bedplates;

- weld order and sequence, including the pre-assembly tack welds;

- weld procedures, methods and positions and thermal pretreatment;

- the ancillary resources needed to provisionally brace the steel plates in each component;

- tools for turning and all other ancillaries required for handling components;

- all the intermediate stages involved in assembling standard components, sub-assemblies or segments.

As the fatigue resistance of a permanent bridge component may be weakened by welded ancillaries, re-usable hoisting and turning tools (Fig. 15) were designed for handling nodes, chords, diagonals and so on with no need for such devices. This proved to be an efficient and effective approach, given the modularity of the structure and the many components with identical outer geometries.

Where lugs were needed to assemble large-scale segments on site, they were consistently defined on shop drawings with 


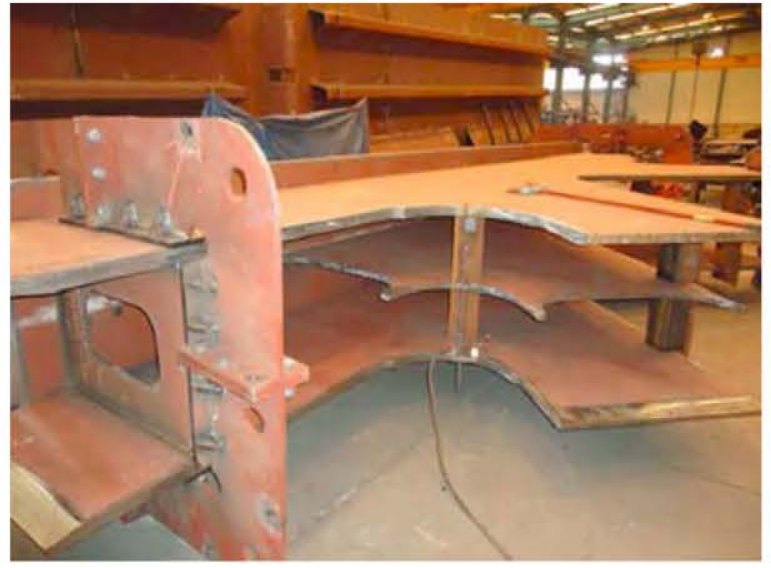

Figure 15. Manufacture of a recumbent node.

suitable fatigue details, dismounted further to specific removal and repair procedures and subject to intense subsequent control.

\subsection{Splicing of standard components and fatigue resistance design}

The connections between chords and nodes were designed to elude the need for four welds on the same cross-section, staggering flange and web connections with a geometry that favoured welding bearing elements prior to loading them with the elements to be borne. As Fig. 13 shows, as a rule the nodes were formed with the end of the bottom flange cantilevered and the end of the upper flange set back. That geometry was reversed in the chords to simplify adjustment and assembly and to avoid establishing four welds on the same section (Fig. 16).

With this geometry, a coupon could be cut into the top flange at the end of the chord to more readily perform three full penetration butt welds with flush-finish dressing inside the cross-section (formed by the webs and the bottom flange). As a result, a fatigue detail category 71 [7] weld with a backing strip was converted into at least a fatigue detail category 80 butt weld dressed to flush [7].

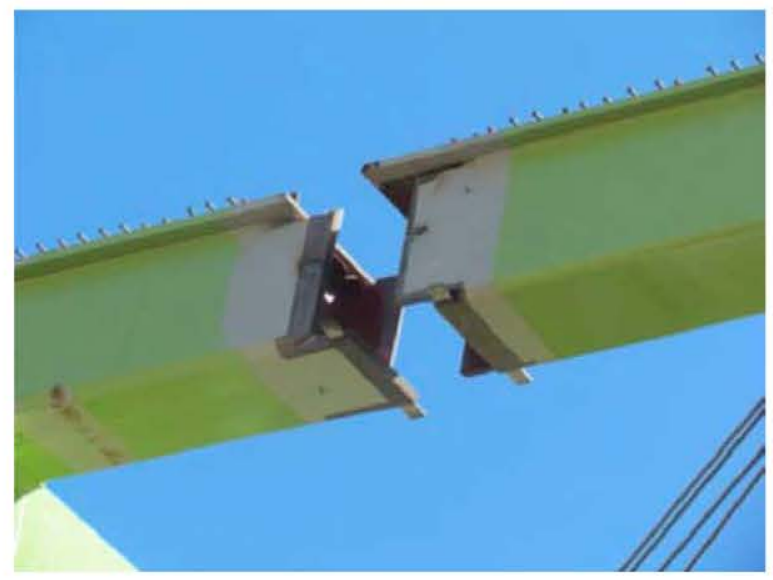

Figure 16. Detail of node-upper chord connection with staggered welds.
Wherever possible, steel structures should be designed without single-sided welds, which indispensably call for the use of steel backing strip. Rather, butt welds should be made from both sides or from one side with provisional ceramic backing strips and subsequent dressing to a flush finish. Butt welds with steel backing strip, in addition to delivering a lower detail category, may generate a series of problems described in greater depth in [6].

As a rule, in a more conventional bridge with composite girders or box girders, such as the Arroyo las Piedras [8] or Archidona [9] composite high speed railway viaducts, the main butt welds in webs and flanges are nearly always made from both sides, obviating the need for single-sided welds with steel backing strip. In trusses, on the contrary, the latter solution must often be adopted, for the connections between nodes and chords and nodes and diagonal web members are often accessible from the outer side only, necessarily requiring the use of steel backing strip.

On the River Ulla Viaduct, although efforts were made to minimise their use, many connections, such as between diagonal web members and nodes (Fig. 13) or the welds joining flanges in node-chord connections, called for steel backing strips. Ref. [6] contains a detailed description of the precautions that must be taken in the performance and control of single-sided butt welds with backing strips to ensure that the connection actually welded is a fatigue detail category 71 according to UNE-EN 1993-1-9 [7].

\subsection{Fatigue in connections and transitions}

In a truss structure with nodes at $15-\mathrm{m}$ intervals, the connection details are recurrent, with the exception of minor variations in the dimensions (thickness) of the plates and the welds. Hence the importance of a suitable approach with a design geared to welds with a satisfactory fatigue detail category.

A few examples of the main repetitive details, in which each node was fully defined on the shop drawings from the outset, are summarised below.

- The drawing on the left in Fig. 17 details the transition for the fillet weld between the flange and web of a diagonal or chord at its interception with a truss node, where the diagonal or chord flange is joined to the web in the truss node with a partial penetration weld, generating a cup hole in both the plan and elevation views, which was remedied with transitions and moderate grinding along the edges of the weld. To ensure suitable fatigue performance, 12 cup holes had to be sealed in each node. To elude the existence of such a large number of sealing strips, which could detract from durability, the solution adopted was a moderate transition with slopes of $1 / 5$ between the fillet weld outside, and the partial penetration weld inside, the centre of the node, as shown in the photograph on the right in Fig. 17.

- The detail for web insertion nodes called for transitioning from two partial penetration but welds with groove preparations on the notched flange only to two partial penetration welds with groove preparations on both the notched flange 

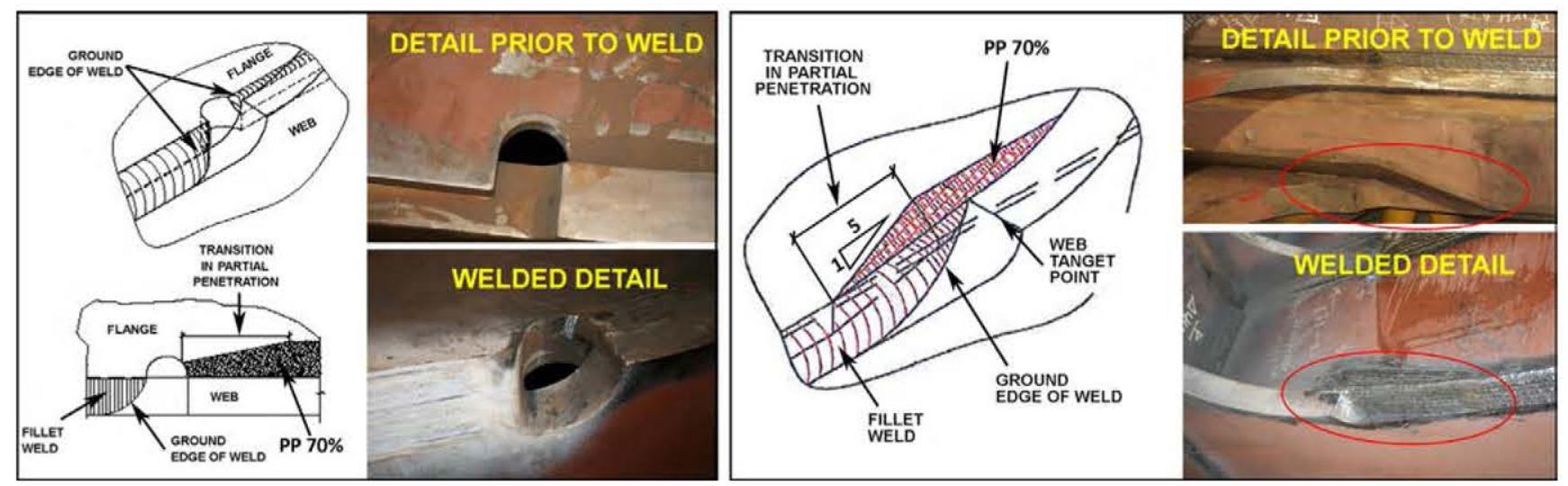

Figure 17. Transition in diagonal or chord flange insertion in nodes: original detail with cup hole (left) and detail with transition and no cup holes (right).

and the edge of the web. That in turn required moderate $(1 / 5)$ transitioning to accommodate the change in the groove, as defined in detail in the shop drawings for each case (Fig. 18).

- Further to detail 3 in Table 8.4 in Ref. [7], to attain a fatigue detail category 80 the edge finish on the webs in web insertion nodes must be tapered and rounded with a radius of over $150 \mathrm{~mm}$ and have a ground tangent weld (Fig. 19).

- Fig. 20 depicts the detail for the end of the weld connecting a web to an upper flange on the bottom node or a bottom flange on the upper node, where the partial penetration weld between notched flange and web was crowned with a tapered, $1 / 5$ slope transition at a cup hole located at the end of the web. The cup hole was subsequently sealed with a plate to ensure node weather-tightness.

- To brace the upper truss chords along the entire deck and the bottom chords in the variable depth area, a bracing plane had to be established with X-braces made of $\mathrm{CHS}$ profiles, welded to gussets in turn welded to the node flanges. Application of this solution, which is more or less conventional in steel and composite decks, to a highly recurrent detail on this viaduct

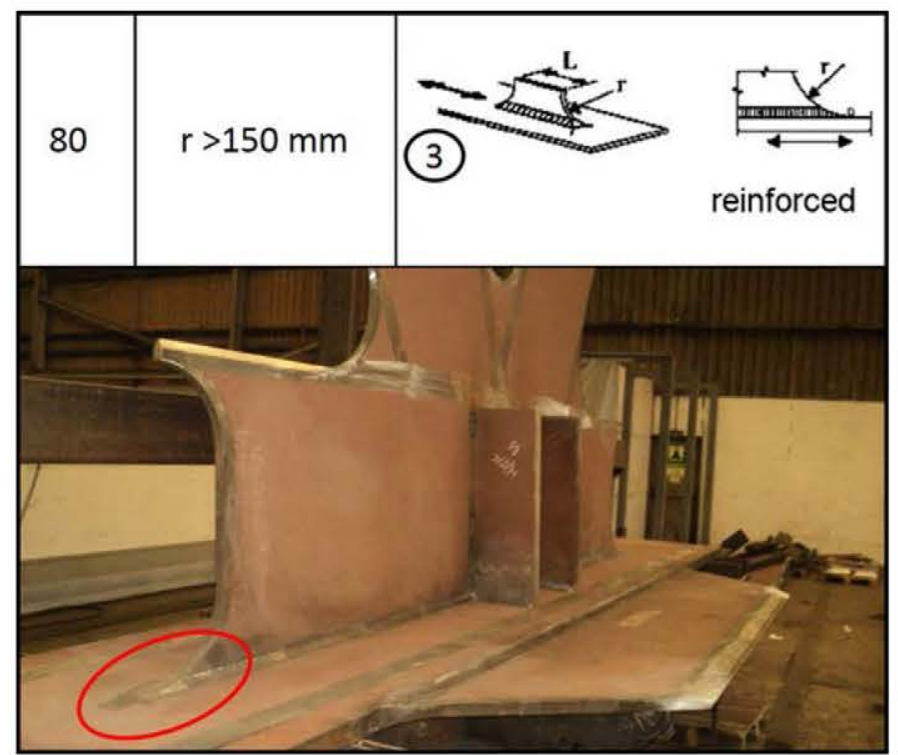

Figure 19. Detail of end of web with ground tangent finish in web insertion node.

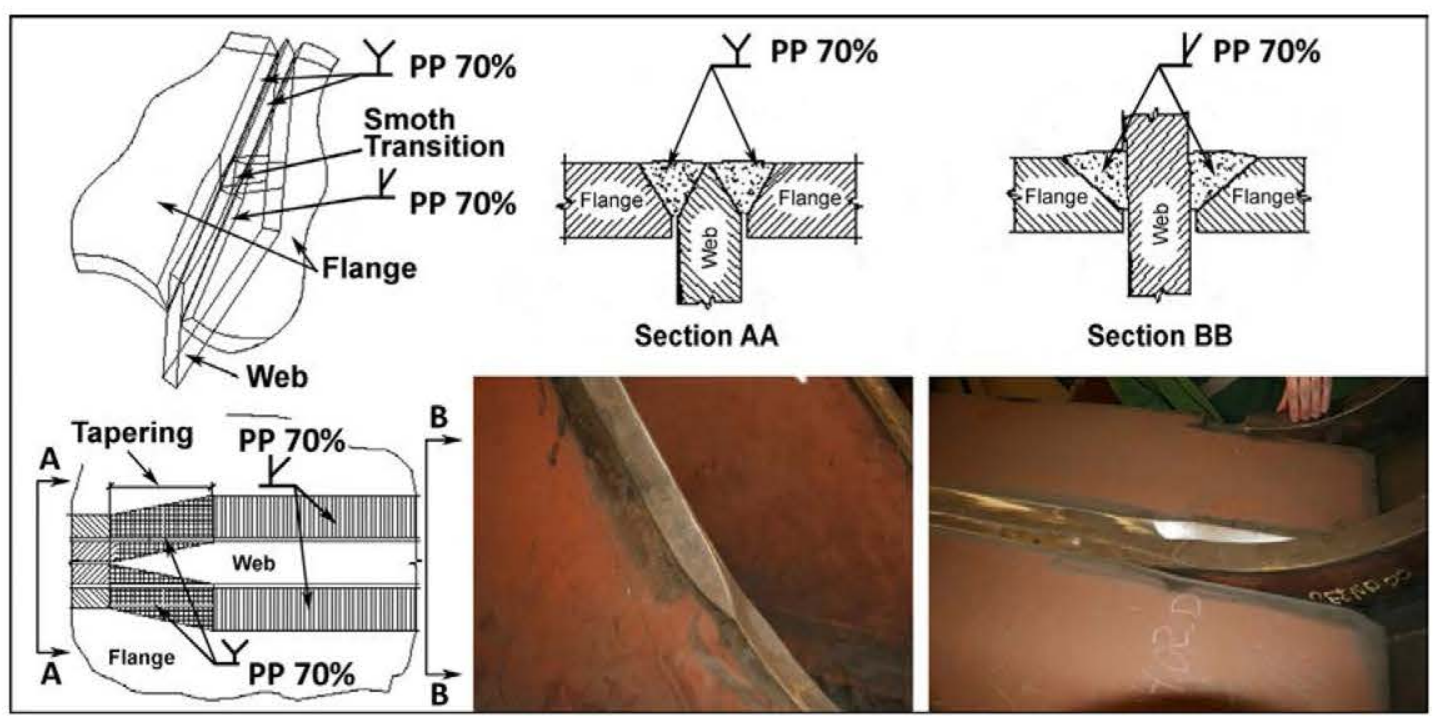

Figure 18. Detail of smooth transitions in groove preparations in web insertion nodes. 


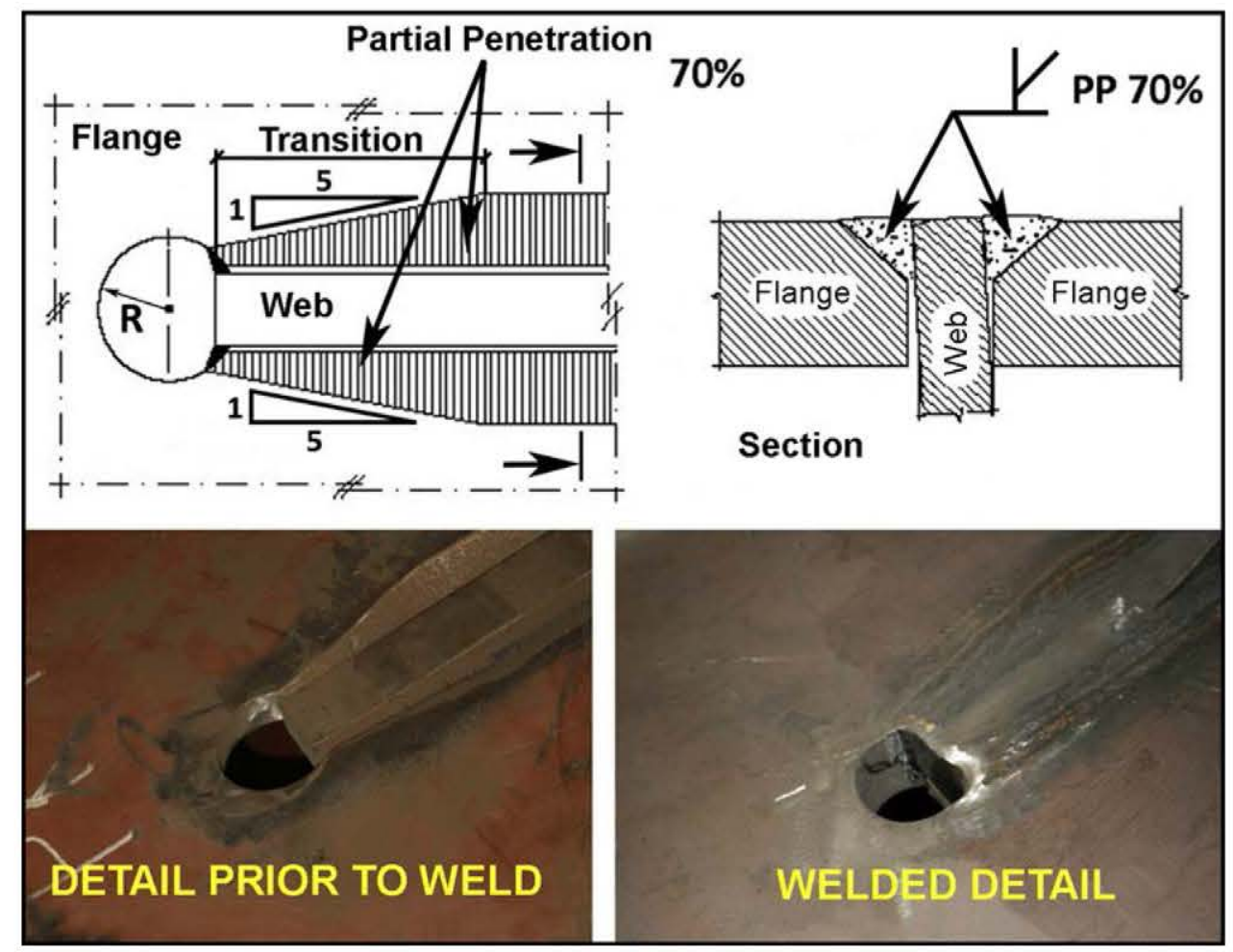

Figure 20. End of web in nodes, cup hole at end of web and weld transitions.

was contingent upon the following precautions to ensure its suitability.

- Further to detail 4 in Table 8.4 in Ref. [7], to comply with a fatigue detail category 90 , gusset edges must be tapered and rounded with a radius of over $150 \mathrm{~mm}$ and the edge of the weld must be ground (Fig. 21).

- Gussets must as a rule be thinner than the main plates on a bridge, in this case on the nodes. Consequently, resorting to the more conventional solution of transitioning from the thicker steel was not judged to be the best approach, for it would have weakened the strength of the main longitudinal section. Here, butt welding the gusset to the flange and transferring the stress on the gusset to the flange more smoothly with an additional fillet weld was deemed to be a more suitable solution (Fig. 21).

\section{Deck construction}

\subsection{Launching gantry construction of left bank approaches}

The design for the steel structure in the four constant depth $(50+80+120+120 \mathrm{~m})$ approach spans on the left bank (Fig. 1), positioned between $\mathrm{A} 1$ and $\mathrm{P} 4$, stipulated that the segments should be hoisted onto provisional shoring where they were to be welded to ultimately form a continuous girder.

After the works were awarded, the River Ulla joint venture studied an alternative procedure. The initial proposal was adapted to use launching technology on spans 3 and 4 (between piers P2 and P4) in light of the scant accessibility of these areas, particularly for segments and cranes, in addition to the presence of two roads underneath the alignment between P2 and P3 and a railway track near $\mathrm{P} 4$.

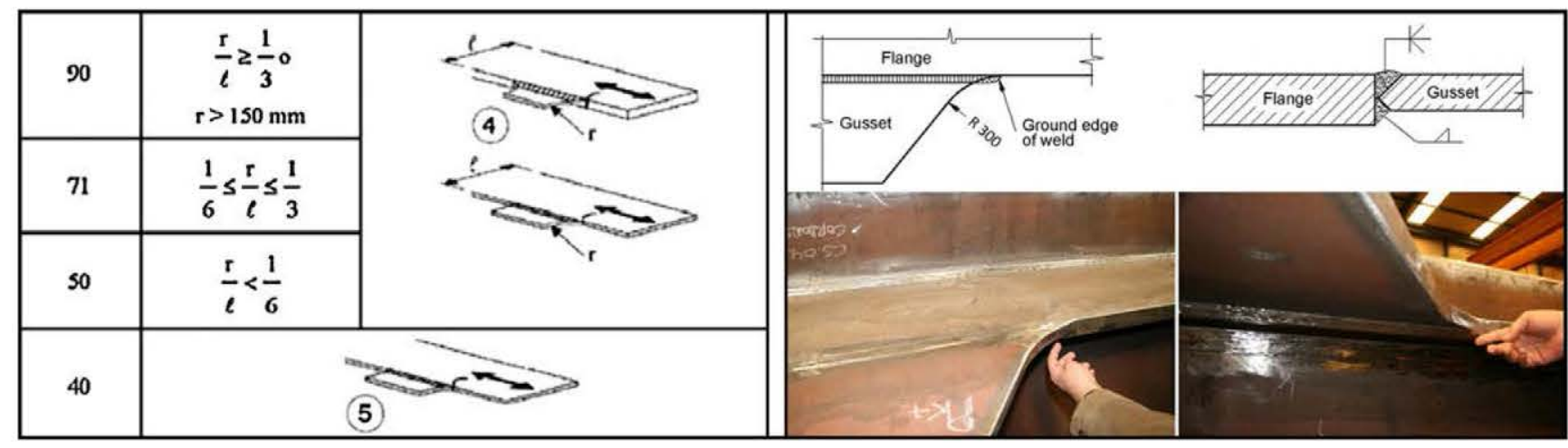

Figure 21. Detail 4 from Table 8.4 in Ref. [7] and tangent weld in a gusset-flange node. 


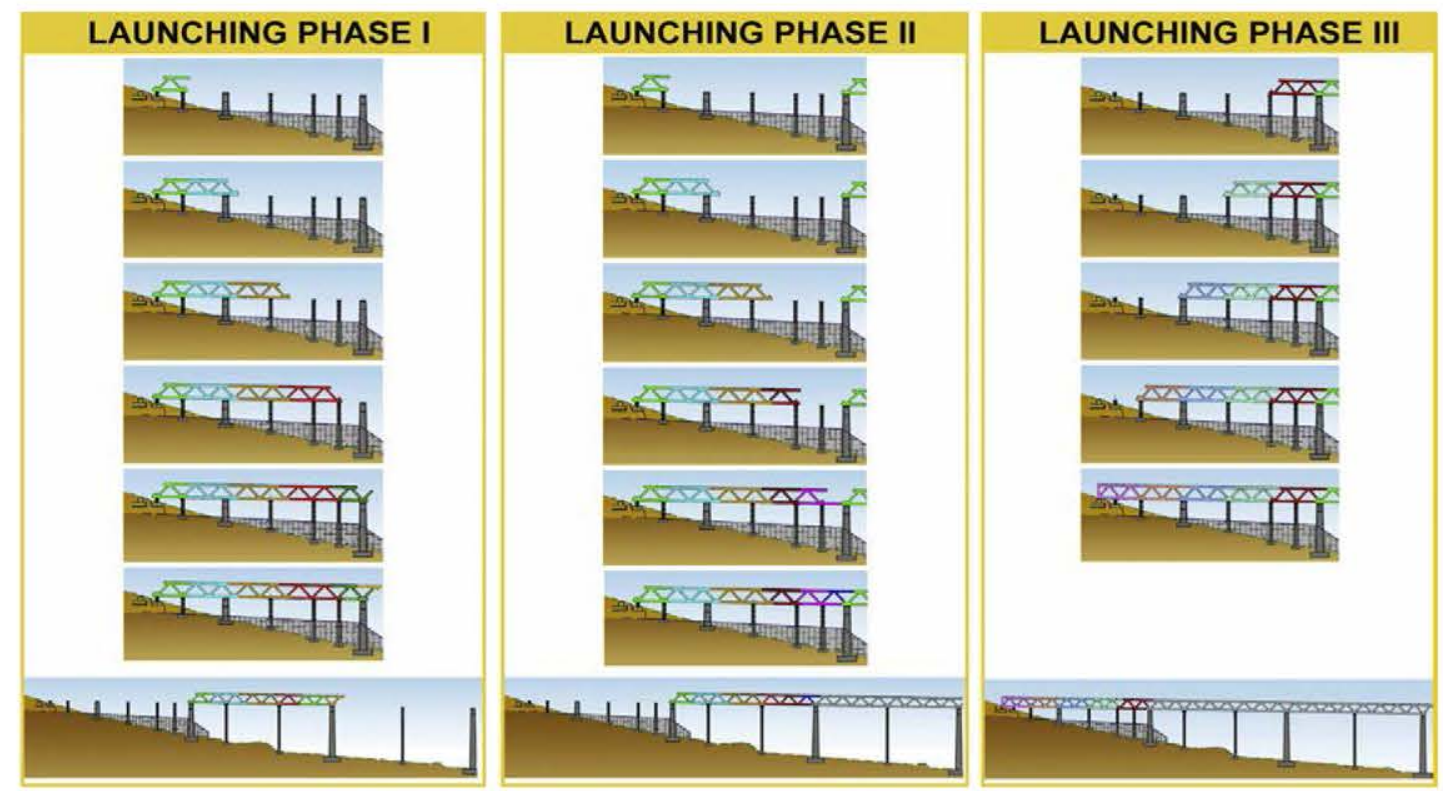

Figure 22. Construction and launching sequences in spans 1 to 4 .

This construction procedure, in keeping with the original design, adopted the general principle of building a continuous girder as the bearing structure and an overhead yard between A1 and P2 for segment assembly, while eluding hoisting operations between P2 and P4.

The launching gantry had to be installed on piers P1 and P2 and provisional overhead shoring was required to avoid interference with a tunnel close to A1 in the preceding section of railway, awarded to another contractor.

Construction on the four spans was divided into three stages, as shown in Fig. 22.

In the first stage, the segments needed to build 120 -m span 4 were hoisted with cranes to the launching gantry and positioned on shoring between $\mathrm{A} 1$ and $\mathrm{P} 2$ and on piers $\mathrm{P} 1$ and $\mathrm{P} 2$. Upon completion, this 120 -m section was launched from P2 to P3 from an ancillary structure anchored to $\mathrm{P} 2$. Given the $-1.8 \%$ grade in this stretch, the section was back-stayed to A1 throughout the operation, during which jacks positioned on P2 pulled on the gradually lengthened back stay cables.

Shoring sited at two intermediate positions between P2 and P3 served as support for the section launched, shortening the length of the cantilever (Fig. 23). Several of the shoring structures between $\mathrm{A} 1$ and $\mathrm{P} 2$ were used for provisional assembly operations only, i.e., not for launching operations per se.

In the second stage, the span 3 segments were hoisted to the launching gantry and connected to the section launched previously by continuous welding. The full $240-\mathrm{m}$ section comprising spans 3 and 4 was then launched from P3 to P4 $(120 \mathrm{~m})$.

The photograph on the left in Fig. 24 depicts the beginning of the second stage, as the section was launched off P3, while the middle photo shows the section in place between P3 and P4 at the end of the operation. An intermediate shoring structure positioned between P3 and P4 reduced the front end span to $60 \mathrm{~m}$. The picture on the right in Fig. 24 shows a crane hoisting the stage 3 segments (Fig. 22) between P2 and A1.

Ref. [10] contains a detailed description of the launching process; the criteria followed to position jacks on some of the provisional shoring to directly or indirectly control the reaction on each bearing throughout the process; the intermediate stages between two launches; the ancillaries needed for the operation, including front jacks and collapsible nose beams, rear jacks, lugs for hooking onto the launching units and back stays, among others; and the controls conducted during the two operations.
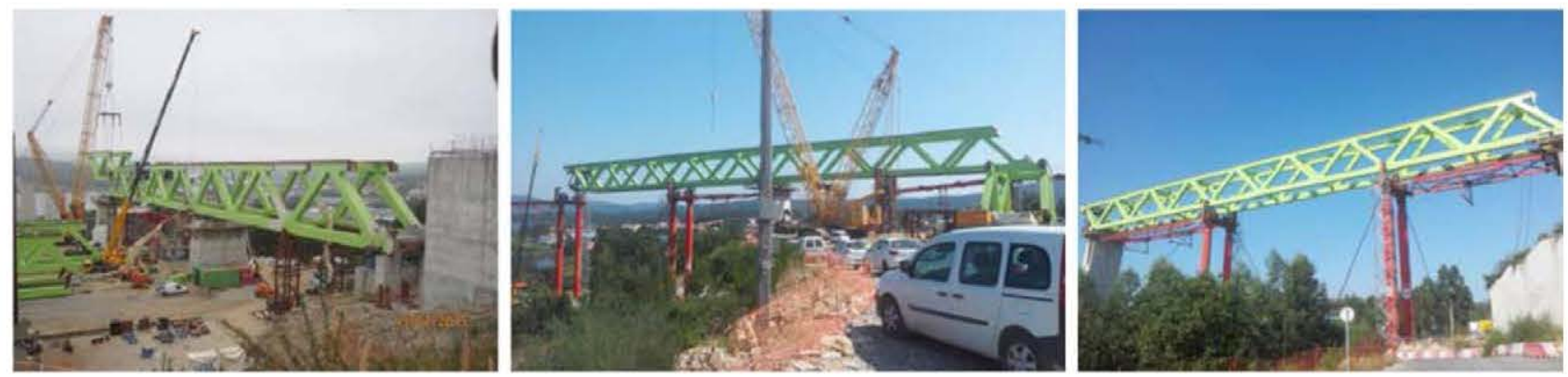

Figure 23. Segment hoisting, first launch and section after launching from P2 to P3. 

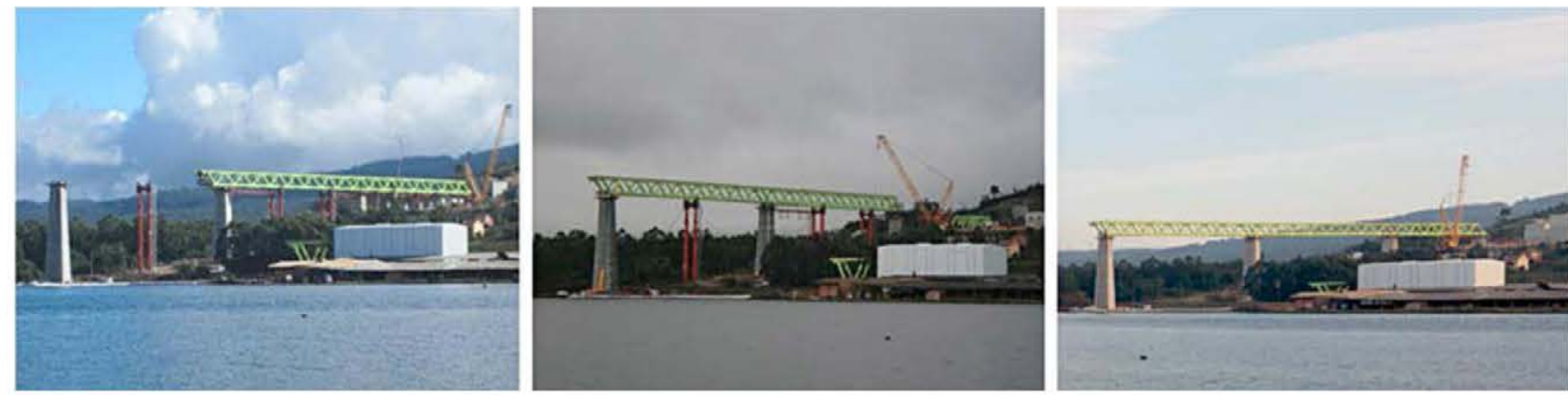

Figure 24. Second stage, 240-m section launched between P3 and P4, and hoisting segments in stage 3.

\subsection{Hoisting of full sections to build right bank approaches}

In the design, the three constant depth approach spans $(120+120+80 \mathrm{~m})$ on the right bank of the River Ulla running between P9 and abutment A2 were to be erected by hoisting segments onto provisional shoring where they would be welded to ultimately form a continuous girder.

In the absence of any hindrance at ground level between P9 and A2, the River Ulla joint venture proposed to adapt the construction procedure and sequence, building the three spans on more readily accessible low shoring platforms directly underneath the final deck location, and subsequent hoisting into place one by one. The construction and hoisting sequences are shown in Fig. 25.

In the first stage, the sections were built underneath the future deck on shoring barely raised off the ground (Fig. 26).

To hoist the 465-t structure for span 12 (Fig. 27), it was provisionally restrained by anchoring the top of segment zero to adjacent abutment A2 and attaching boxes filled with sand to the bottom as an arrester against the abutment [11]. Due to its delta shape and hinge at its provisional bearing, the zero segment on the P11 end was temporarily restrained (from the time it was positioned until immediately prior to hoisting) to prevent overturn. The hoisting cables were then hooked to the end of span 12 and secured with back stays to the heavier preceding section to prevent overturn during hoisting, during which the provisional torque restraint was released.

The hoisting and staying reactions as well as segment zero geometry were controlled during hoisting.

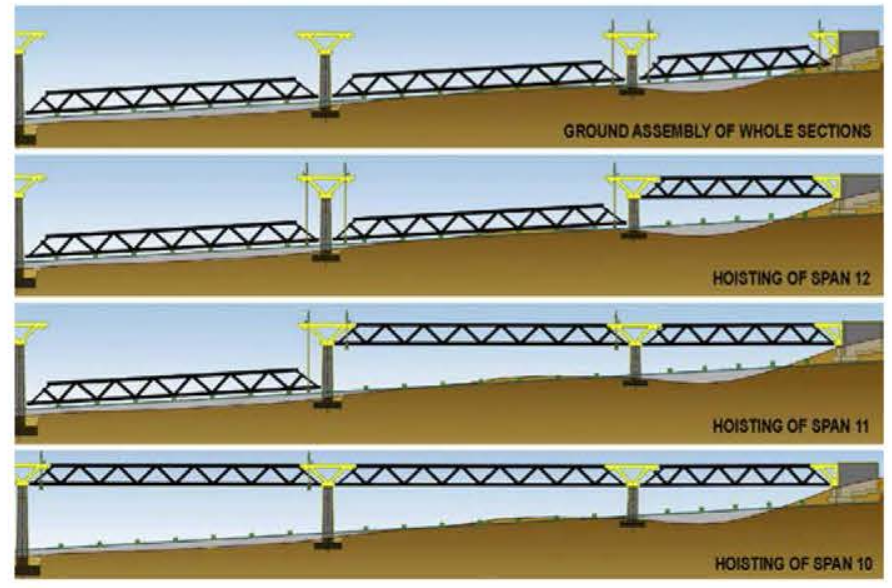

Figure 25. Hoisting sequence for approach spans 12, 11 and 10.

After the first section was hoisted, its zero segments on both ends were provisionally secured and thus braced it was welded into position, after the connections were duly fitted. Reels with extra cable were designed for each connection and positioned at the ends of the chord and diagonal for quicker and easier alignment of the components for overhead connection.

With the first section in place and segment zero on the second in position at P10, span 11 was hoisted between P10 and P11 (left photograph in Fig. 28). As this 900-t, 120-m long (centres) section had to be offset on the span 10 side with a section of similar characteristics and weight, to secure segment zero on P10, a counterweight had to be used to enhance safety and prevent possible overturn of the pierhead segment during hoisting.
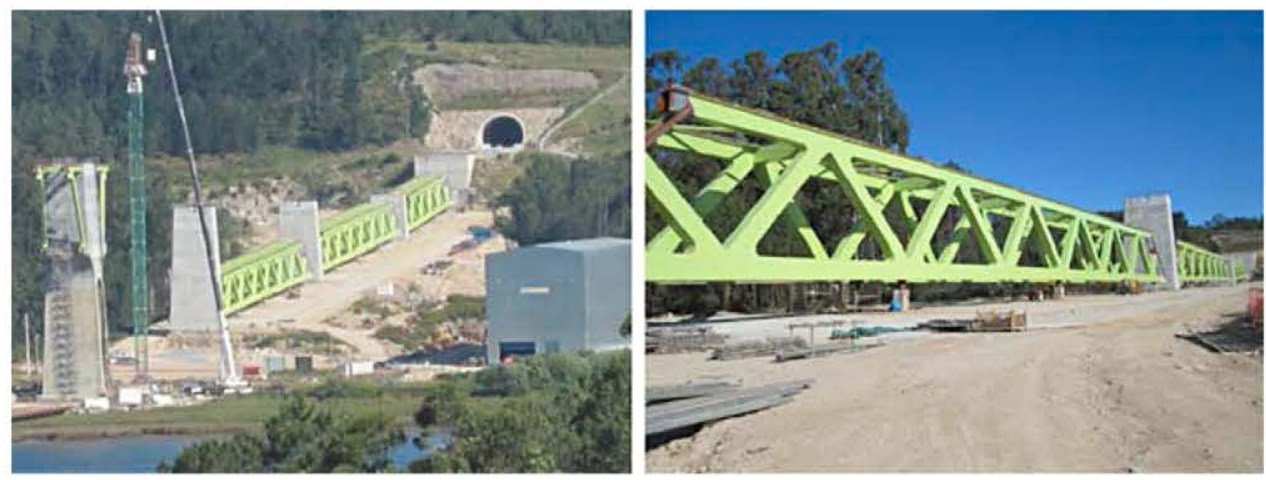

Figure 26. Sections for spans 10-12 built on the ground, ready for hoisting. 


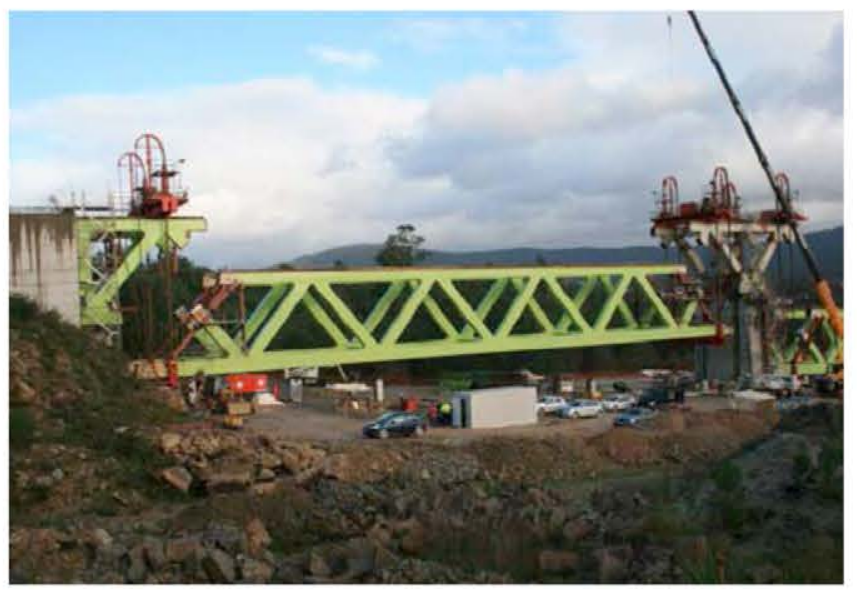

Figure 27. Hoisting sequence for 465-t approach span 12 between A2 and P11.

In the third and last hoisting operation, the 965-t section running between $\mathrm{P} 9$ and $\mathrm{P} 10$ was raised from both ends and joined by continuous welding to the two adjacent spans (photograph on the right in Fig. 28).

See Ref. [11] for a detailed description of the hoisting process for these three spans and the ancillaries needed.

\subsection{Balanced cantilevering construction of variable depth spans}

Due to the environmental protection afforded the estuary, the five variable depth centre spans, measuring a total of $930 \mathrm{~m}$, were erected with balanced cantilevering techniques from the four centre piers, as envisaged in the design (Fig. 29).

The large size and heavy weight of the steel 'W'-shaped zero segments in each pierhead added to construction complexity. Each side of the 375-t segment measured $35 \mathrm{~m}$ long and $17.5 \mathrm{~m}$ high. The standard components shipped from the plant were assembled with each side of the segment face down on the platforms resting on the sheet piles at the foot of each pier (left photograph, Fig. 30) used to build the foundations. Once all the components were welded, the two sides were uprighted simultaneously with a large (25-m high) provisional ancillary structure positioned on the pierhead (right photograph in Fig. 30). This complex operation involved simultaneously jacking the top members of the zero segment upward and sliding the bottom members, propelled by jacks and connected to skid shoes, toward the pier on tracks (Fig. 30).

The two uprighted sides of the segment were balance-hoisted (left photograph in Fig. 31) to the pierhead elevation. To position these two huge elements in their permanent location they had to be skidded inward, secured to a pin connection at a lower leve and subsequently rotated from the head of each side to slope each half-segment on a grade of $1 / 17.5$. Once in their permanent position, the two sides were inter-braced at the upper tie girders and their bottom chocks were concreted (right photograph in Fig. 31).

The concrete pierheads were subsequently cast, after which the piers and zero segment were ready for balanced cantilevering to commence.

Beginning at the zero segment, the steel structure to be cantilevered was divided into full width, $15-\mathrm{m}$ long segments, with a $7.5-\mathrm{m}$ stagger between the ends of the upper and bottom chords to facilitate overhead assembly. The following segments were cantilevered off piers P5-P8 (Fig. 32).

- Six segments were built in span $5(\mathrm{PK}-)$ and six in span 6 $(\mathrm{PK}+)$ from pier P5.

- Six segments were built in span 6 (PK-) and six in span 7 $(\mathrm{PK}+)$ from pier $\mathrm{P} 6$.
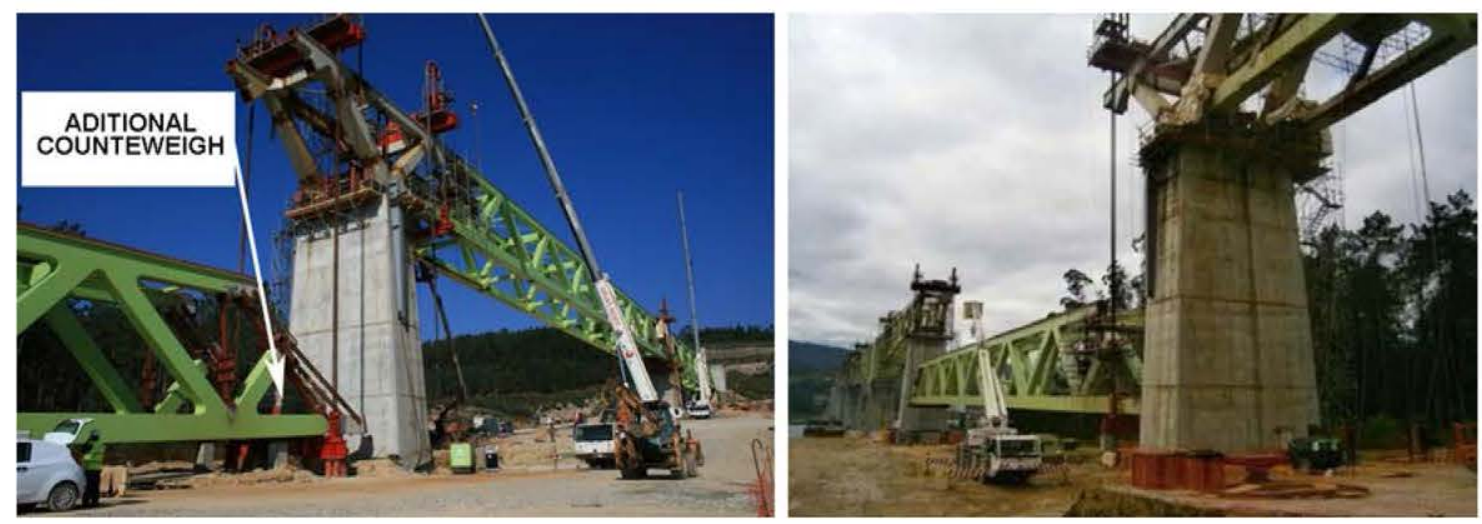

Figure 28. Hoisting sequence for spans 11 (left) and 10 (right).

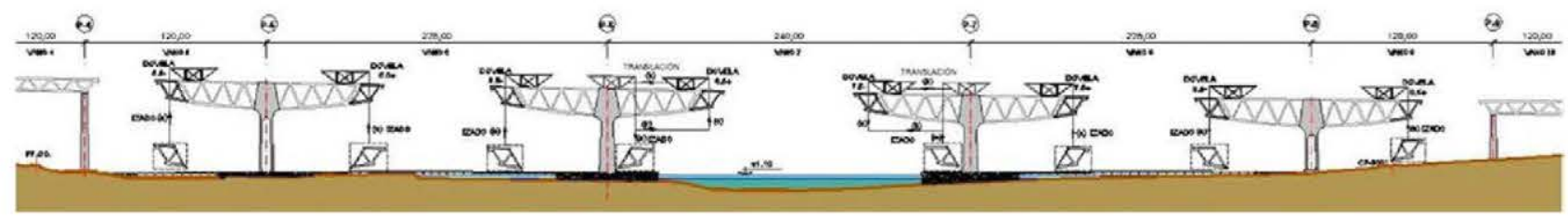

Figure 29. Balanced cantilevering in the five variable depth centre spans. 

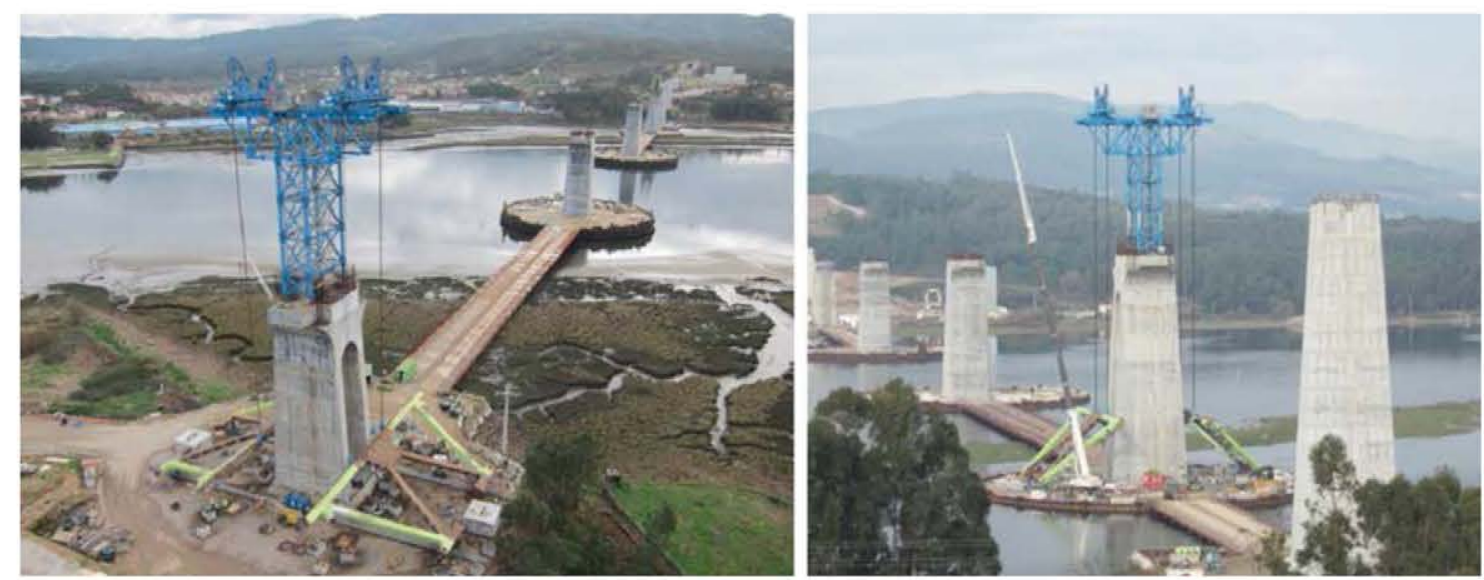

Figure 30. Zero segment construction and uprighting at the foot of the pier.
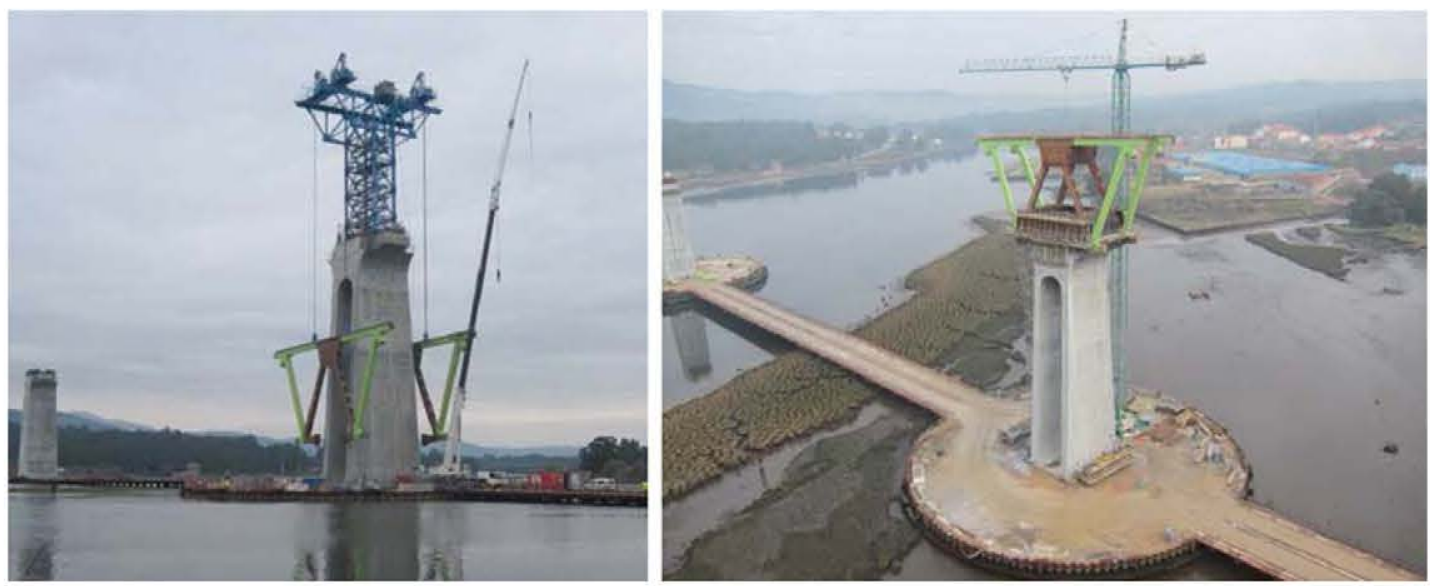

Figure 31. Hoisting zero segment and positioning on pierhead.

- Seven segments were built in span 7 (PK-) and six in span 8 $(\mathrm{PK}+)$ from pier $\mathrm{P} 7$.

- Six segments were built in span $8(\mathrm{PK}-)$ and six in span 9 $(\mathrm{PK}+)$ from pier $\mathrm{P} 8$.

\subsubsection{On-site variable depth segment assembly and transport}

The variable depth segments were assembled in two of the three assembly bays sited alongside the river banks (middle and right-hand photographs in Fig. 14). The segments measured nearly $17.50 \mathrm{~m}$ high (the biggest ones) by $7.10 \mathrm{~m}$ across at the top and nearly $10 \mathrm{~m}$ at the bottom and $22.5 \mathrm{~m}$ long from the nose of the upper chord to the rear of the bottom chord. They weighed a maximum of $300 \mathrm{t}$ each.
In light of their enormous size, for readier construction and shipping, the variable depth segments were divided into the following standard components: rear-cantilevered upper chord, front-end upper node, front-end diagonal and cantilevered rear diagonal, bottom node and cantilevered bottom chord, tie girders and cross-braces for upper and bottom nodes and chords (Fig. 33).

The segments were assembled on a production line two-bytwo and trial assembled (Fig. 33) to ensure perfect alignment of the six ends after hoisting. Where physical trial assembly was not performed, the segments were joined virtually with computer software to ensure the same degree of precision and accuracy.

The elements on the 'open' segments (with staggered upper and bottom nodes [12]), i.e., the cantilevered rear of the upper chord, the cantilevered diagonal springing from the bottom node

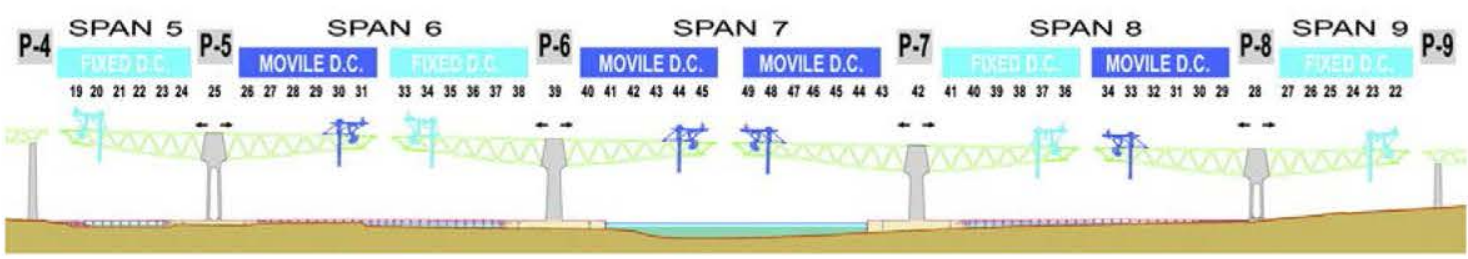

Figure 32. Division of the five variable depth spans into segments, showing derrick cranes. 

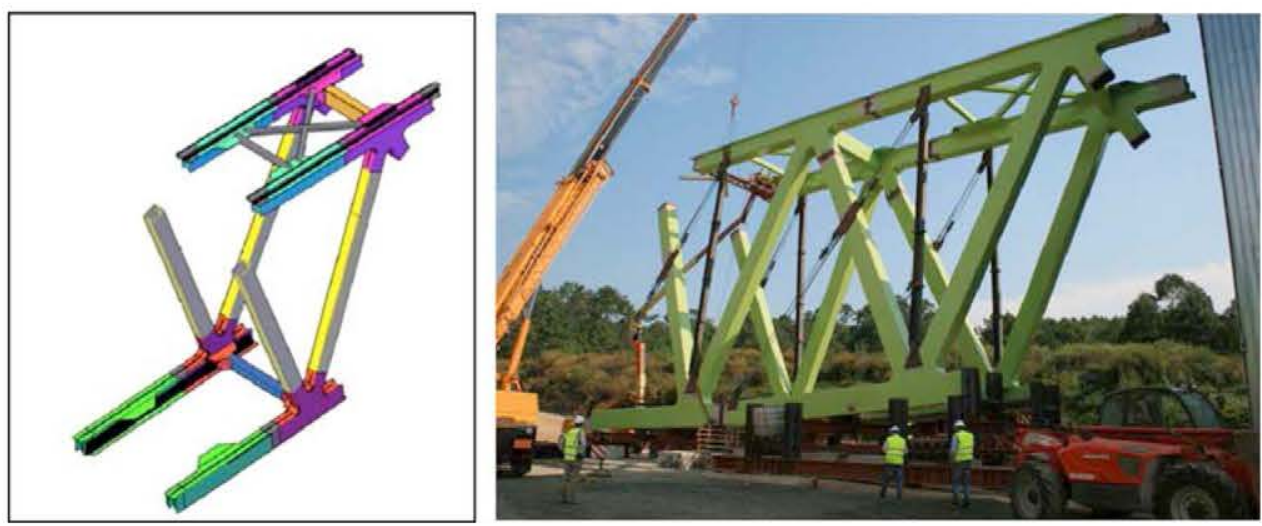

Figure 33. Segment breakdown into components and two segments assembled on the ground.

and the cantilevered bottom chord, staggered $7.5 \mathrm{~m}$ beyond the upper chord (Fig. 33), had to be provisionally secured with specially designed braces. These devices controlled strain during transport and hoisting and adjusted the cantilevered ends of the diagonal and rear of the bottom chord for alignment with the preceding segment.

The variable-depth segments were transported on site on two separate (one for each side of the segment) self-propelled, multiwheeled platforms (Fig. 34).

Span 9 was directly accessible by land and all the span 5, 6 and 8 segments could be hoisted from the wharves (Fig. 29). The section in the centre span between P6 and P7 was the sole balance-cantilevered member that was inaccessible from land.

One of the possibilities studied during the design and early into the works stage was to carry the two arms of the span 7 section in barges. That operation had to be ruled out, however, due to the difficulties involved. Ultimately, the segments for the centre span were hoisted into place over the estuary with mobile derrick cranes, as described in the following section.

With this solution, the variable-depth span sections were built with no contact with the ground below and hence without affecting the main riverbed, the marshes or the plant life on the bank, further to the project specifications.

\subsubsection{Derrick cranes for segments}

This part of the deck was built with balanced cantilevering techniques first from P5 and P8 and subsequently from centre piers P6 and P7. This called for designing and manufacturing four derrick cranes, two stationary and two mobile, on which to position a hydraulic jack-based hoisting system.

The 'stationary' derrick cranes were so called because as they hoisted the segment from a position anchored to the deck (Fig. 35), they carried no load other than their own self-weight when travelling.

The mobile derrick cranes (Fig. 36), in turn, could collect a segment at the base of the pier, hoist it partially, carry it parallel to the direction of the bridge while suspended (underneath the existing cantilever) to its location at the end of the section under construction and then hoist it into its permanent position for welding to the preceding segment. As these mobile cranes were needed in the centre span between $\mathrm{P} 6$ and $\mathrm{P} 7$, two stationary and two mobile derrick cranes were designed and manufactured.

Since piers P5 and P8 were accessible from the ground or the provisional wharves, there the mobile derrick cranes operated like their stationary counterparts, hoisting the segments vertically while anchored to the deck, with no need to move suspended segments in any other direction than upward.
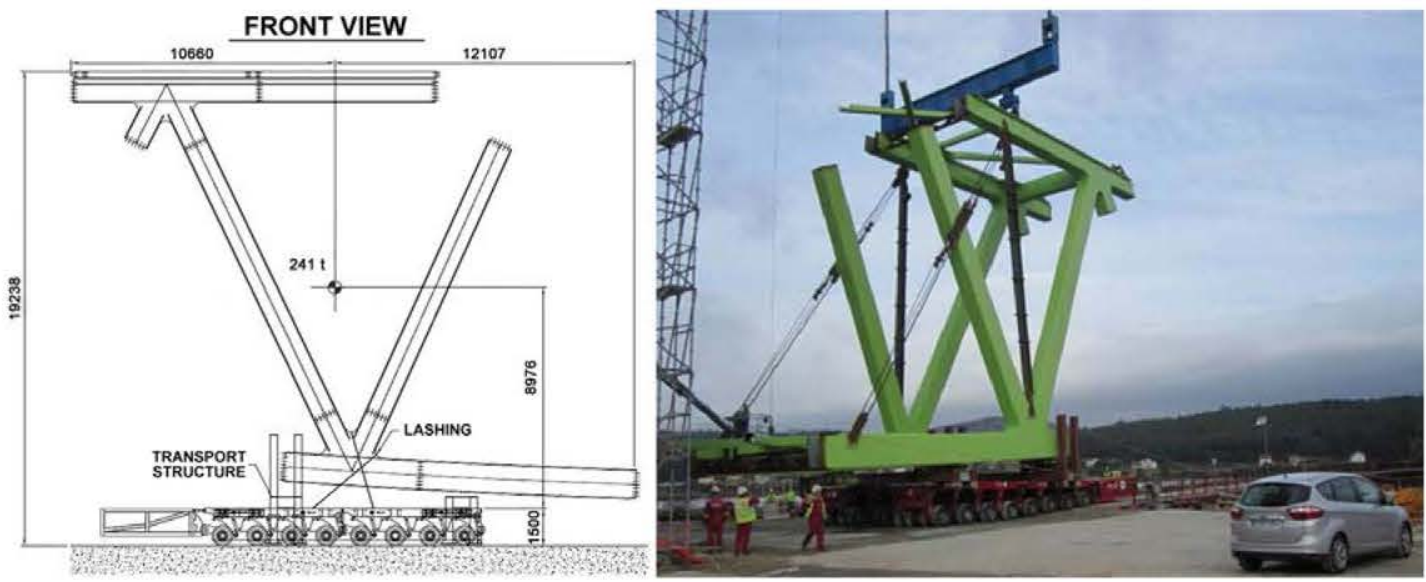

Figure 34. Drawing and photograph of self-propelled platforms. 


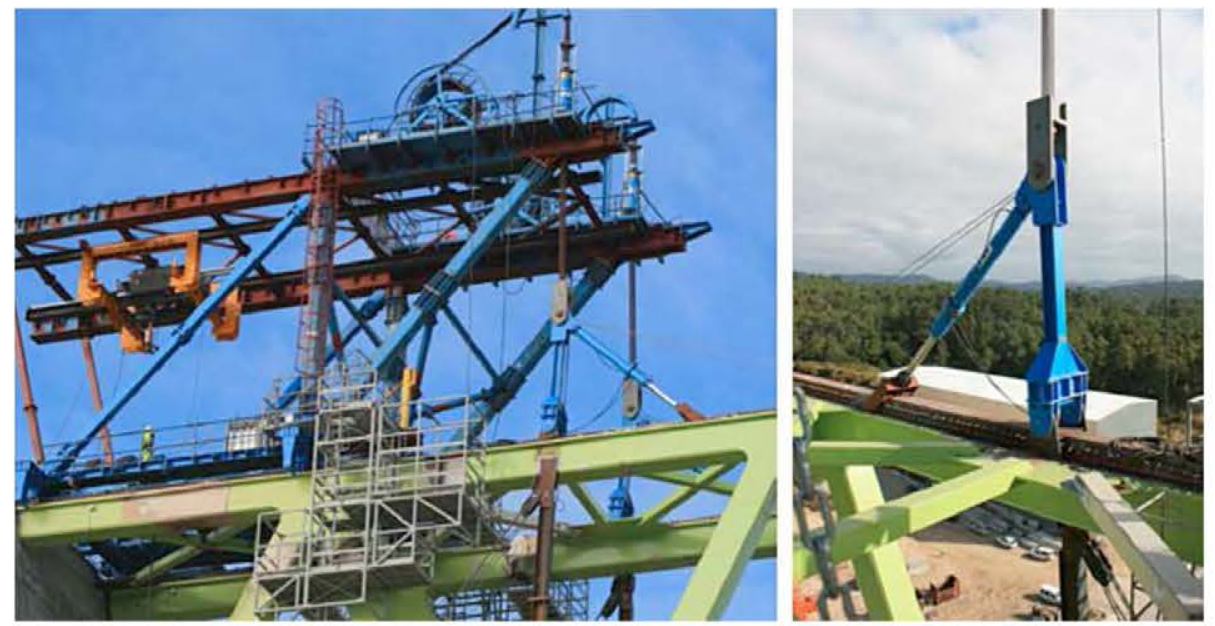

Figure 35. Hoisting a segment with a stationary derrick crane and detail of transverse hoisting beam.

Hydraulic jacks propelled the derrick cranes over the truss on skid shoes over tracks resting on lubricated neoprene-Teflon bearing pads positioned on the closed profile crowning the upper chords.

The stationary derrick cranes were fitted with hoisting jacks that were aligned vertically with the hoisting lugs on the segments (Fig. 35) very near their centre of gravity. Once hoisted to the desired elevation, the segments were aligned with a transverse hoisting beam fitted with a slanted jack (Fig. 35) hooked to the alignment lugs, which were located at in the front end. When the alignment jack was released, the segment rotated, bringing the bottom chord into position, whereas when the jack piston was
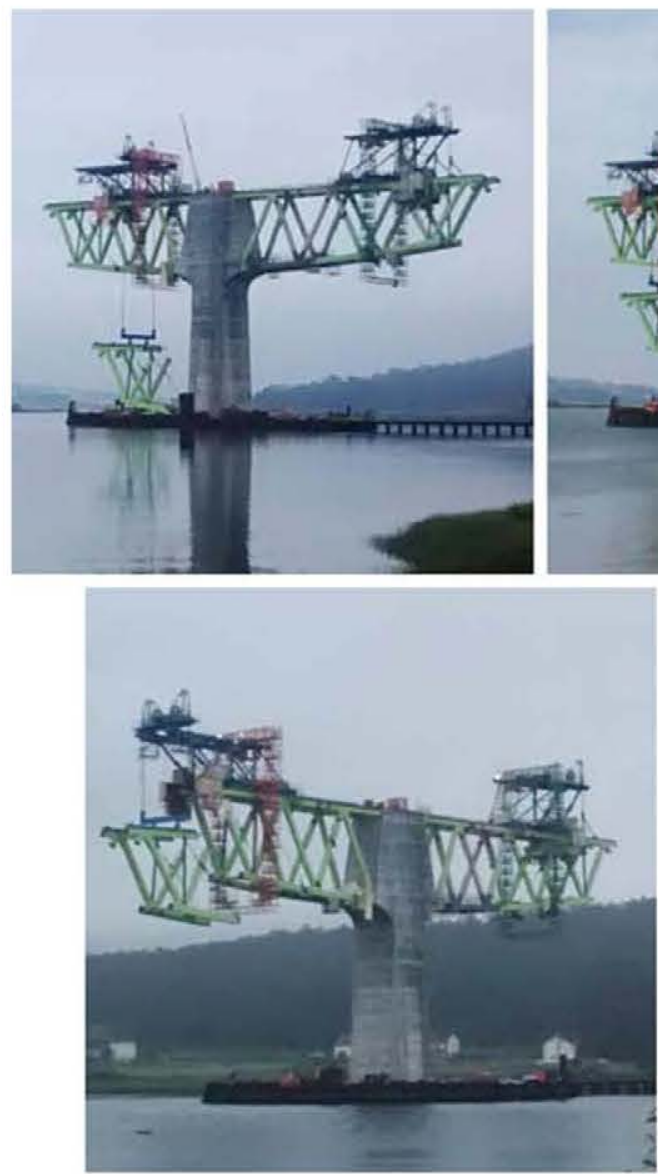
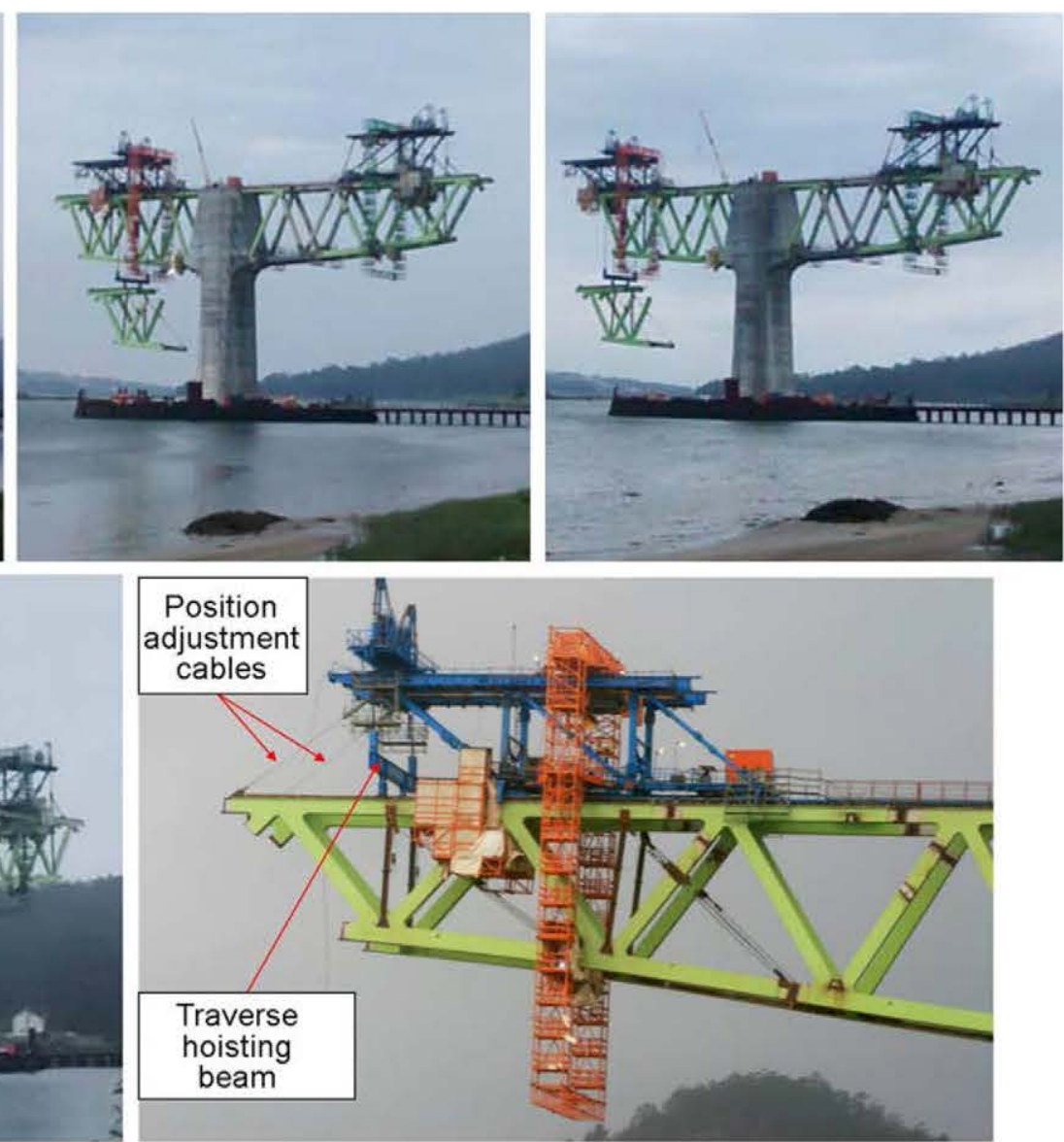

Figure 36. Hooking, hoisting and moving a segment with a mobile derrick crane; hoisting to permanent elevation and details of the transverse hoisting beam and segment alignment cables. 

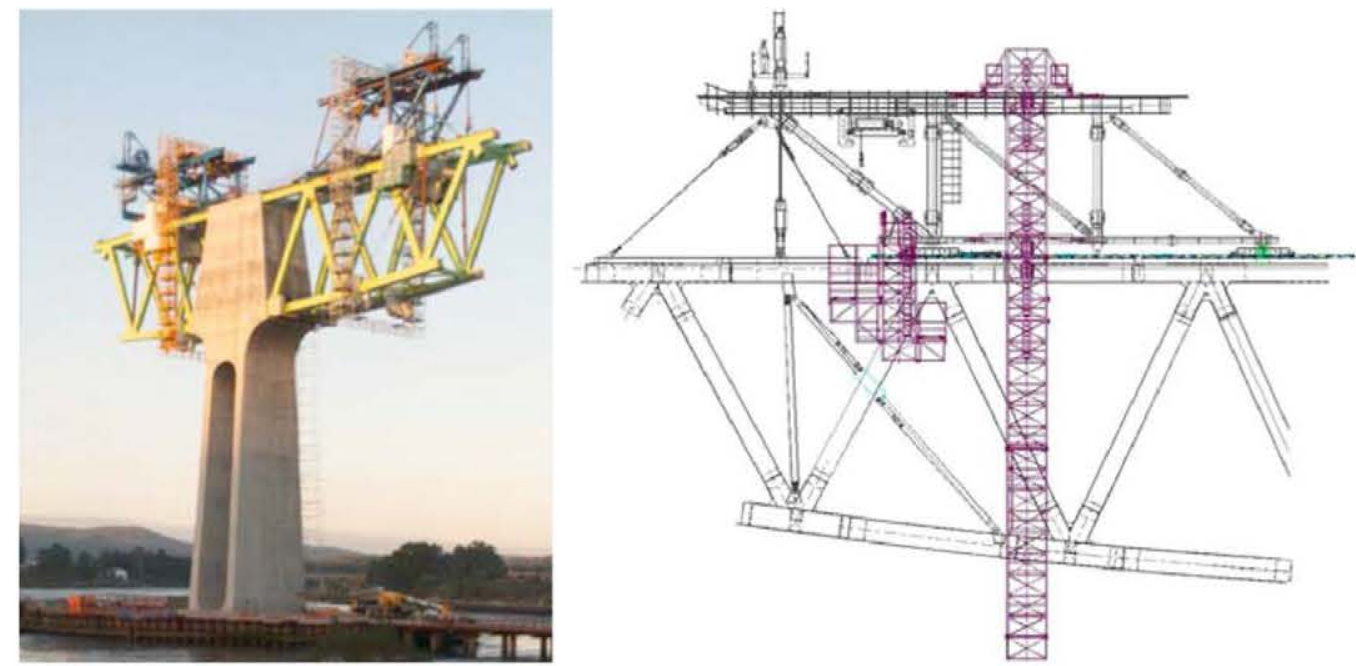

Figure 37. Photograph and drawing of access platforms and protected areas for welding.

closed, the segment rotated in the opposite direction, moving the bottom chord away from its position [12].

The main difference between the mobile and stationary derrick cranes was that in the former the hoisting jacks and cables could not be aligned vertically with the hooking lugs positioned on the top of the segment when carrying suspended segments in a horizontal direction. Rather, the cables had to be realigned outward with a cross-beam located on the derrick crane, and a transverse hoisting beam had to be placed between the segment and the hoisting cables (Fig. 36). Once the segment was at its specified elevation, alignment cables were secured to the lugs located on one end of the element for minor re-positioning prior to welding [12].

\subsubsection{Overhead segment alignment and welding}

When the segments were nearly in position, the accuracy of their alignment was verified topographically prior to welding to the preceding segment. Positioning involved the use of hoisting jacks, the transverse hoisting beam (in stationary derrick cranes) or stiffening cable tensor jacks (in mobile derrick cranes). As necessary, manual tensioners or jacks were used.

Overhead adjustment and welding were performed on specifically designed welding platforms protected from the wind and rain. Each derrick crane was fitted with four welding platforms: two at the front for adjusting and welding the upper chords, diagonal web members and braces (Fig. 37); and two at the rear, with longitudinal and vertical mobility to adjust and weld the bottom chords and braces at different elevations, depending on deck depth.

The front welding platforms were fitted with removable inner components that had to be in position to access the inner side of the chords and removed for the crane to move to the next position. The provisional stiffening was removed from the rear welding platforms when the crane was in motion.

Broadly speaking, the sequence to position a hoisted segment was as follows: assembly of the inner components on the front platforms, alignment and welding of the upper chord and upper cross-bracing, alignment and welding of the bottom chord and diagonal, change in rear welding platform position, coupling and welding of the bottom cross-brace, welding of the crowning profile on the upper chord, partial removal of the front welding platforms, and lastly, unhooking of the hoisted segment and travelling forward to the next hoisting position.

The photographs below show balanced cantilever progress from P5 (Fig. 38) and several stages of progress from other piers (Fig. 39).

\subsubsection{Joining balanced cantilevers}

With the exception of span 9, each pair of variable depth sections was joined by a segment with a special geometry. This segment was divided in two: an upper chord that was welded to the end nodes in the two adjacent cantilevers, and a lower element, consisting in two bottom chords, the mid-bottom node and two cantilevered diagonals springing from the bottom node.

The construction process was as follows in all cases.

- The bottom of the segment was hoisted and aligned with one of the two ends. The bottom chord and diagonal on the other end were fitted with cable on a reel for more accurate longitudinal alignment.

- The segment was provisionally secured with ancillary elements on the end opposite the chord and diagonal and the length of the cable was subsequently adjusted as needed to align and weld the joining segment.

- During the night, the ends of the two upper nodes were simultaneously secured with provisional tools to subsequently position the upper chord of the closing segment with the aid of adjustment cables on reels on both ends.

- Once all the components were welded and tested, the securing ancillaries were removed and the derrick cranes were released, closing the balanced pair.

The sequence followed to join the spans was as follows. 

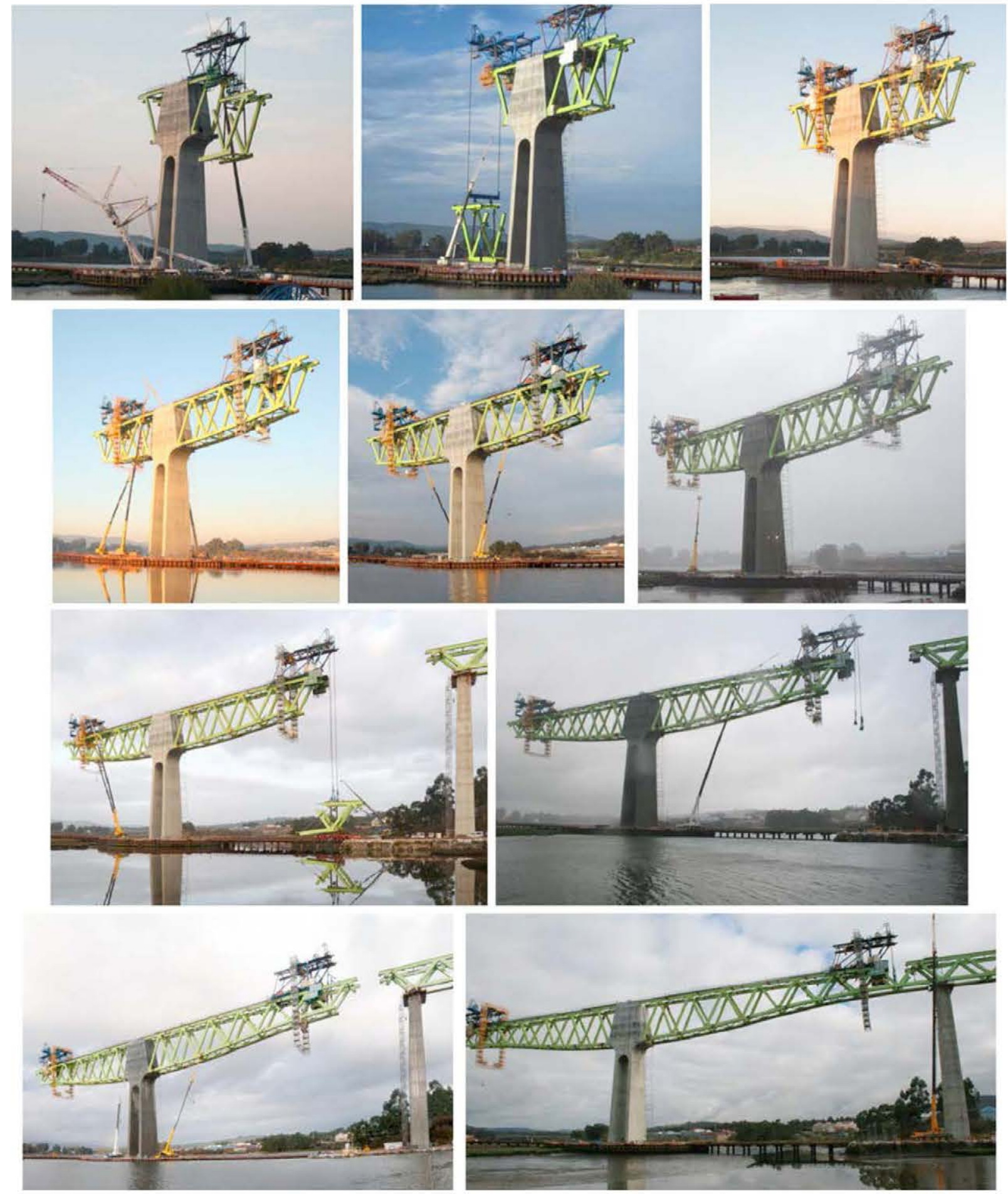

Figure 38. Balanced cantilevering from pier 5 (in foreground).

- On the P4 end of span 5, the segment was hoisted with the stationary derrick crane from the PK- end of the cantilever (Fig. 40).

- In span 9, a double segment was hoisted from the ground with cranes, one end was rested on P9 and the other welded to the PK+ side of the cantilever (Fig. 41). Span 10 was hoisted fully assembled from the end of the segment located on the pier (photograph on the right in Fig. 28).
- In span 6 the segment was hoisted with the stationary derrick crane from the PK- end of the P6 cantilever (Fig. 42).

- In span 8 the segment was hoisted with the stationary derrick crane from the $\mathrm{PK}+$ end of the P7 cantilever (Fig. 43).

- The closing segment for the $240-\mathrm{m}$ centre span (span 7) was hoisted with the mobile derrick crane from the $\mathrm{PK}+$ end of the P6 cantilever (Fig. 44). 

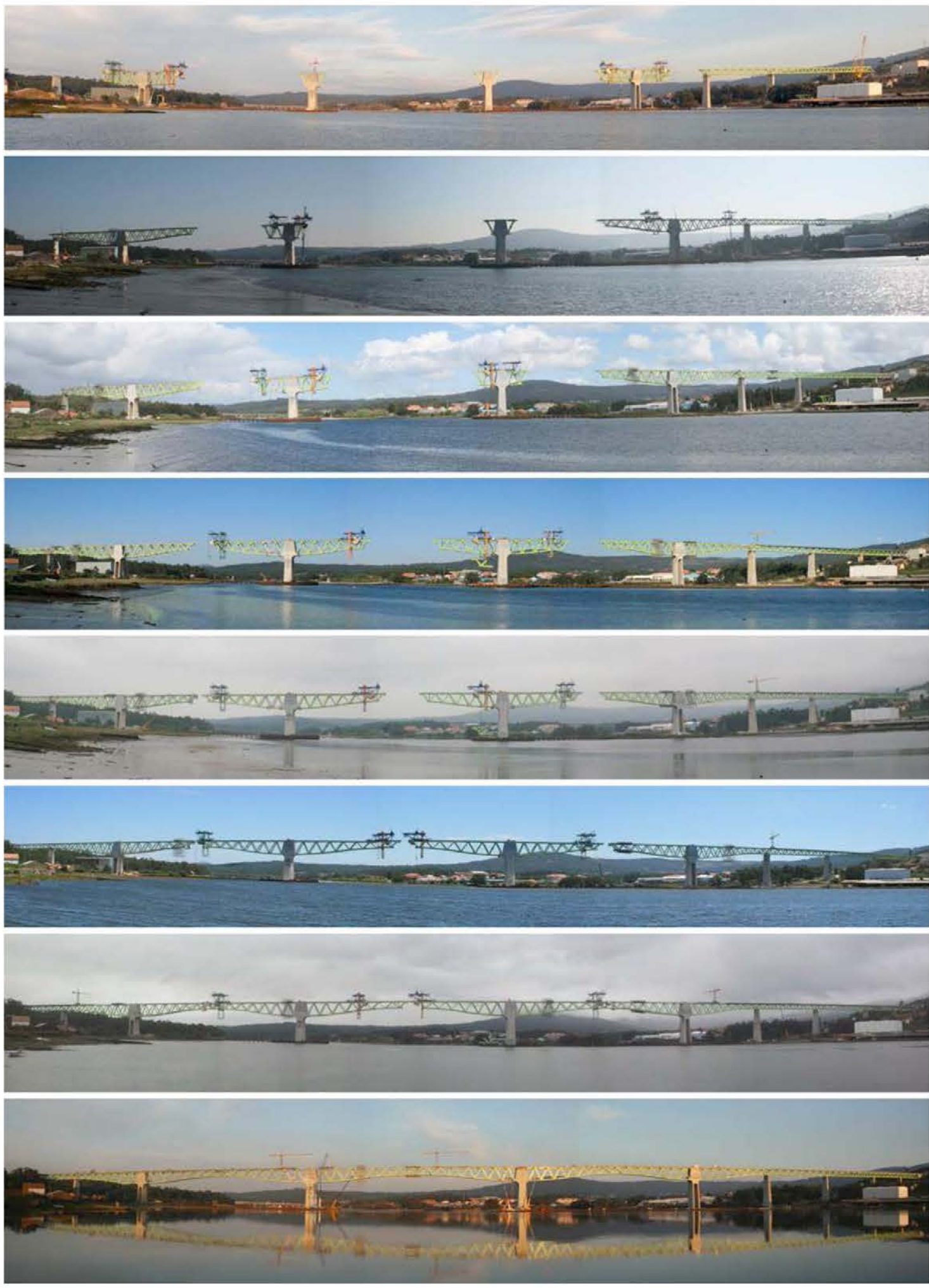

Figure 39. Balanced cantilevering from P5 (right) and P8 (left) and subsequently from P6 and P7.

\subsubsection{Casting the bottom and upper slab concrete}

Once spans 5 and 9 were completed and the deck structure was continuous from $\mathrm{A} 1$ to $\mathrm{P} 5$ and $\mathrm{A} 2$ to $\mathrm{P} 8$, the bearings on piers $\mathrm{P} 4$ and $\mathrm{P} 9$ were lowered by $25 \mathrm{~cm}$ in simultaneous and strictly controlled operations. The aim was to reduce the portal effect-induced bending moments on piers P5 and P8 resulting from the huge difference in the span lengths (225 and $120 \mathrm{~m}$ ) on either side of these two main piers. Lowering these bearings reduce the decompensated bending moments from the permanent loads on piers $\mathrm{P} 5$ and $\mathrm{P} 8$. 

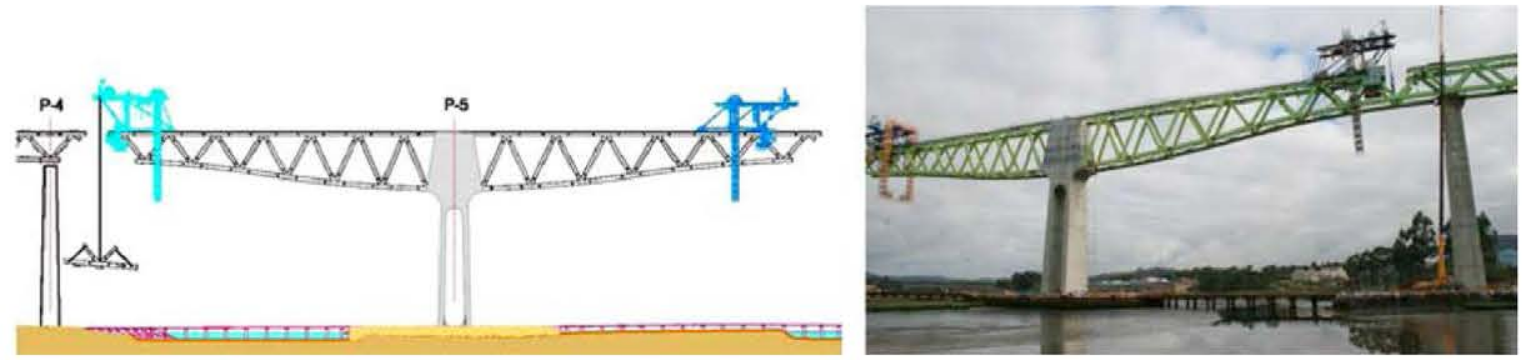

Figure 40. Closing span 5 from a stationary derrick crane.

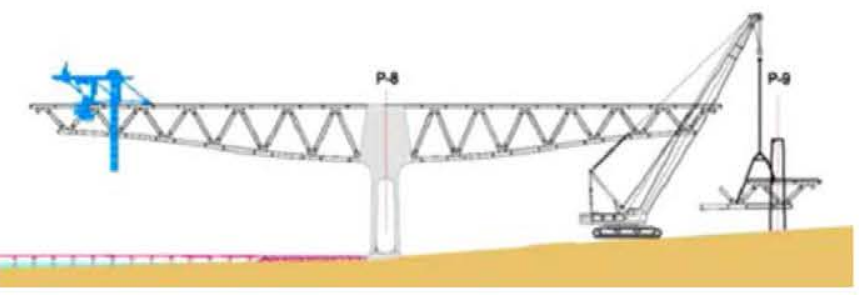

Figure 41. Closing span 5 with a stationary derrick crane and span 9 with a crane from the ground.

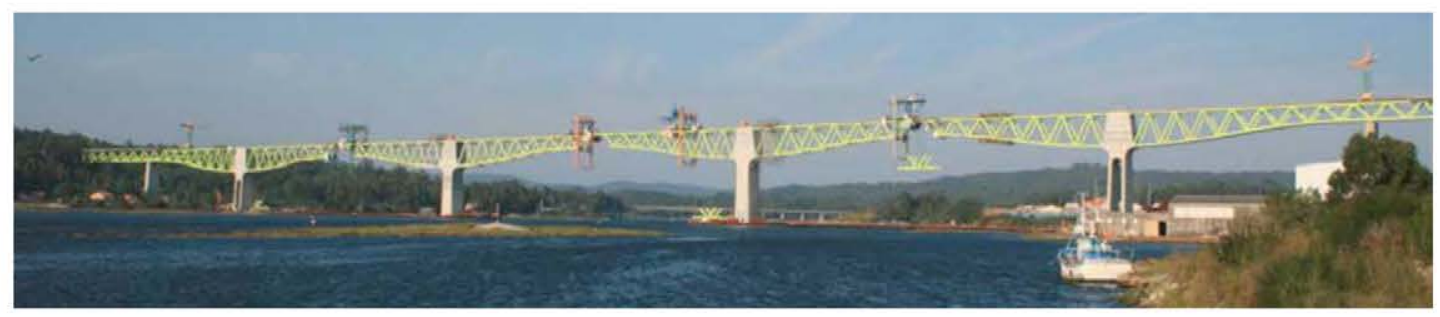

Figure 42. Hoisting the joining segment for span 6.

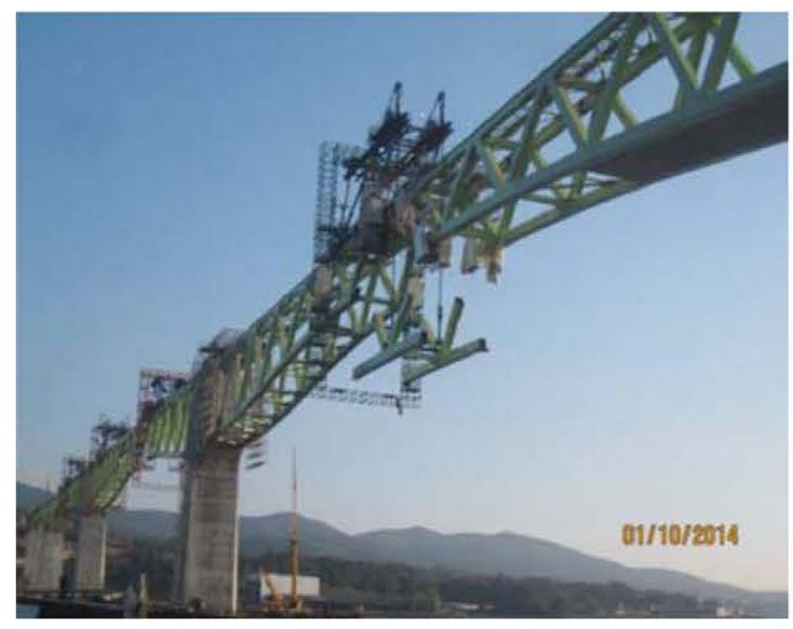

Figure 43. Hoisting the joining segment for span 8.

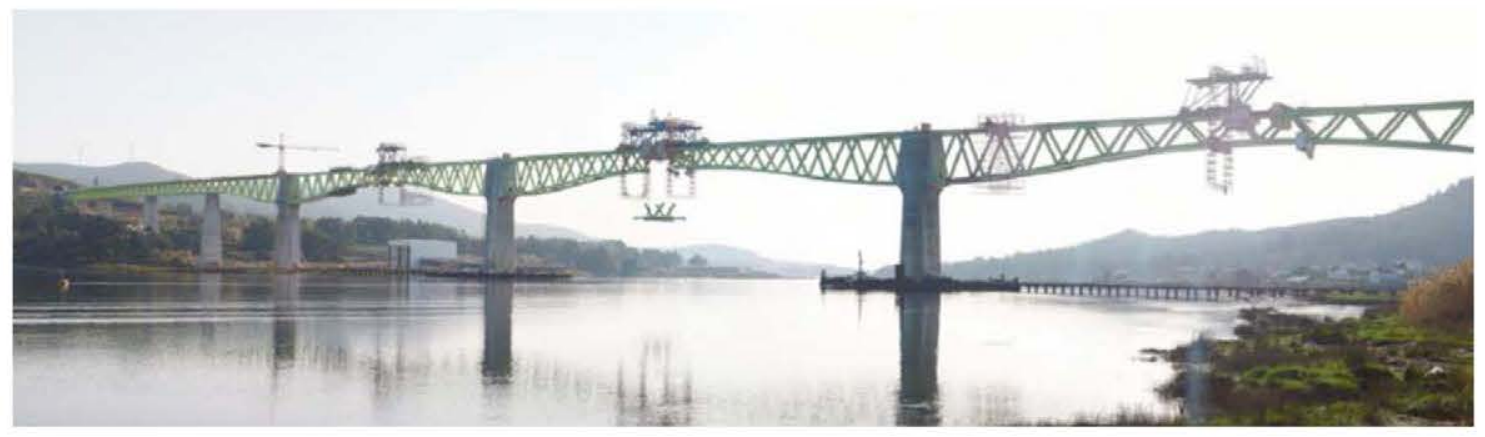

Figure 44. Hoisting the joining segment for span 7. 


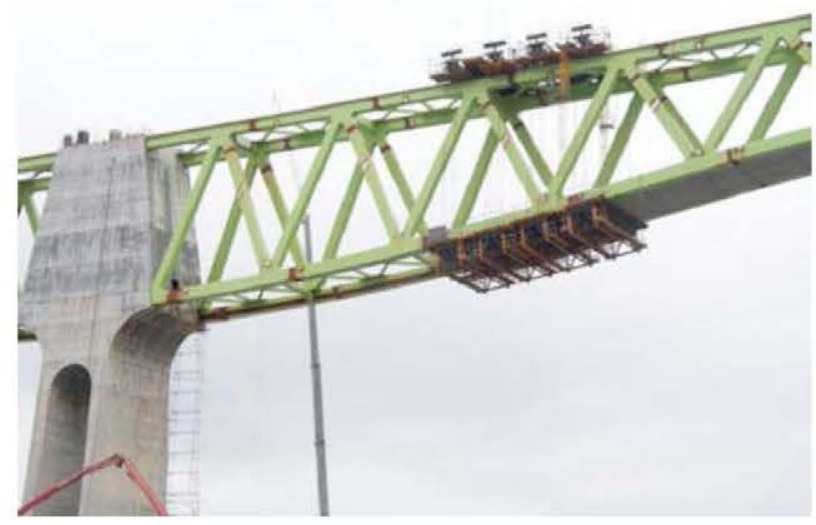

Figure 45. Travellling formwork for casting bottom slab concrete.

Lagging somewhat behind steel structure assembly, concrete was cast on the lower deck slab across the entire bridge. Composite structural concrete was cast in negative moment areas, while in positive moment areas unconnected to the truss, concrete was laid merely to ensure access to deck interiors for inspection purposes. The travelling formwork used to cast the bottom slab concrete in both the constant and variable depth areas of the viaduct (Fig. 45) hung from an upper ancillary structure attached to the crowning profile on the upper chords.

The upper slab concrete was cast on full-width precast slabs positioned with a tower crane mounted on the steel deck itself. This operation lagged behind both the steel structure assembly and bottom slab casting (Fig. 46).

Pursuant to standard practice in composite bridges with reinforced slabs, the upper slab was cast to a sequence that avoided self-weight-induced cracking in hooging areas (Fig. 46). Continuous watering and geotextile protection were deployed to prevent drying shrinkage-induced cracking in the slab.

\section{Conclusions}

With the commissioning of the high speed Atlantic railway between Pontevedra and A Coruña in spring 2015, the viaduct over the River Ulla (Figs. 47 and 48) set a new world record for span length in composite high speed truss typology bridges. Its three $225+240+225-\mathrm{m}$ spans are longer than the 208-m span on the Nantenbach Bridge, which had held the record since its completion in 1993.

As noted in the foregoing, a number of circumstances make the River Ulla viaduct a unique structure, including:

- the use of three very complex construction processes: hoisting in place of whole spans, incremental launching and balanced cantilevering in the main spans;

- the use of unique ancillary resources manufactured explicitly to build the viaduct;

- the use of large-scale (up to $68 \mathrm{~m}$ in diameter) sheet pile enclosures to dry-build the riverbed foundations;

- the face-down assembly of the zero segments for the centre riverbed piers (measuring $35 \mathrm{~m}$ long by $17.5 \mathrm{~m}$ high and weighing $2 \times 375 \mathrm{t}$ ), followed by complex and unprecedented uprighting and hoisting operations;

- the use of high thermo-mechanical performance steel uncommon in Spanish bridges (S-460-M and S-460-ML);
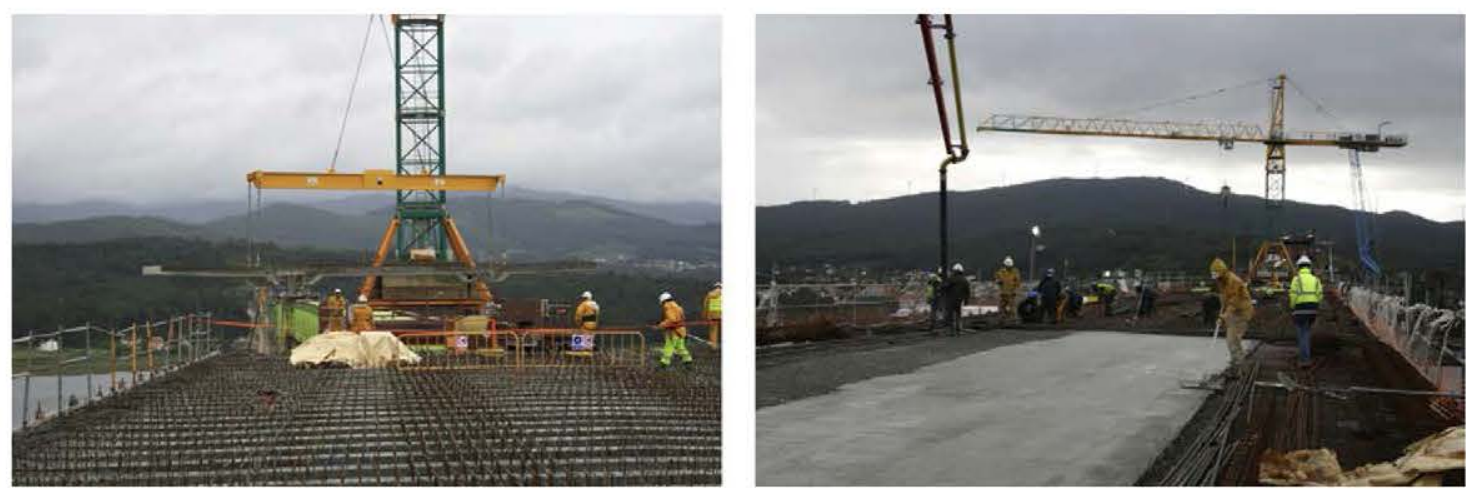

Figure 46. Laying full-width precast slabs and concrete casting on the upper slab.

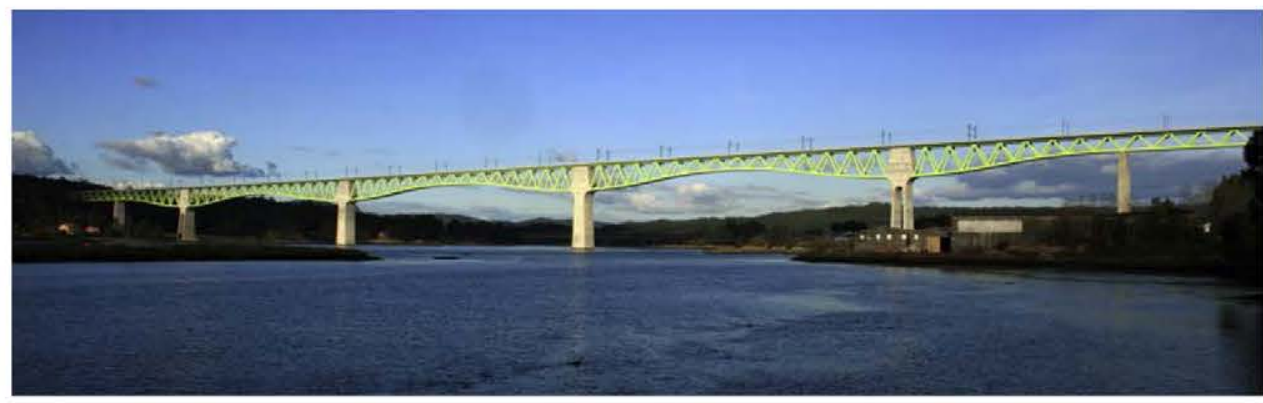

Figure 47. Finished viaduct. 


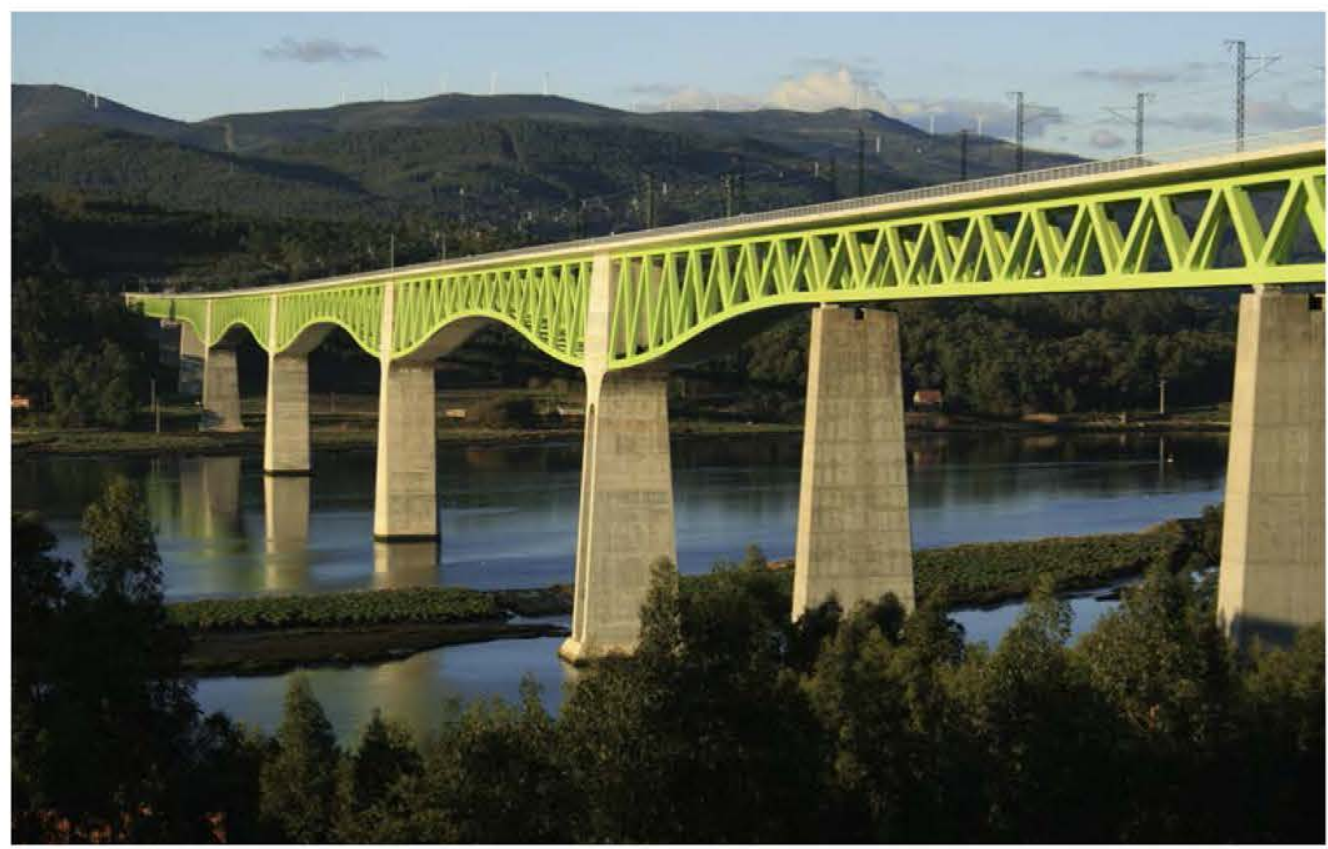

Figure 48. Finished viaduct, side view.

- the construction of three large on-site segment assembly bays with an indoor clearance of $20 \mathrm{~m}$;

- the on-site transport and hoisting of 300-t segments up to $17.5 \mathrm{~m}$ tall with self-propelled multi-wheeled platforms;

- the use of high-strength (RC-70) and self-consolidating concrete on the centre piers;

- the $>70-\mathrm{h}$ uninterrupted casting of over $5000 \mathrm{~m}^{3}$ of concrete in foundations, with thermocouple-controlled setting temperatures;

- the deployment of a standard-setting steel structure quality control system unprecedented in Spain for controlling steel and composite bridges and viaducts.

These unique features earned the River Ulla viaduct the 2015 edition of the Construmat award for the best civil works, 2015 edition of the San Telmo award, price awarded by the Galicia Demarcation of the College of Civil Engineers, and the accesit of the VII Aqueduct of Segovia 2015 prize awarded by the College of Civil Engineers and Fundación Caminos.

\section{Appendix A. Main participants in the design and works}

\section{River Ulla viaduct design}

Developer:

Directorate General of Railways. Ministry of Internal Development

Design manager: Ángel Lanzarote

River Ulla Viaduct conceptual and detailed design:

IDEAM: Francisco Millanes, Miguel Ortega, Luis Matute, Carlos Gordo, Jesus Martín, Alicia de Frutos.

Technical assistance for design drafting:

UTE IDEAM-Euroestudios

Author of the design: Francisco Millanes (IDEAM)

\section{Construction of River Ulla viaduct}

Developer and Site Management:
ADIF Alta Velocidad/Directorate General of Railways, Ministry of Internal Development

Site Managers: Rubén A. Estévez (ADIF AV)/Marina Soler (Ineco) Technical assistance, site management:

TYPSA: Luis Conde, Carmen Casas, Mar Cariño, Jaime Losada Specialised structural consultants for the site management and its technical assistance team:

IDEAM: Francisco Millanes, Miguel Ortega, Pedro Atanasio, Adrián Sánchez, Juan Luis Mansilla, Jokin Ugarte, Carlos Gordo.

Site management's steel structure quality control body:

Applus.

Site management's quality control body for the rest of the works:

Enmacosa

Builders:

UTE río Ulla. Dragados-TECSA

Manager: Pedro Chico/Jorge Sánchez; Site supervisor: Imanol Güemes; Steel structure construction coordinator: David Mouriño; Quality supervisor: Susana Cuevas

Builder's technical assistance

Dragados Technical Services: Luis Peset, Felipe Tarquis, Susana López Manzano, Pilar Hue

Fhecor, consultant engineers: Hugo Corres, Julio Sánchez, Javier de Cabo, Miriam Alonso

Steelworks:

Ascamón-Joama/Martifer/Emesa/Dizmar

Builder's steel structure quality control bodies:

Alfainstant and AMT

Spherical bearings:

VSL

Launch of spans 3 and 4 , and hoisting of segments in variable spans 5 , 6, 7, 8 and 9:

VSL

Hoisting of spans 10, 11 and 12 and on-site segment transport: ALE

Foundations subcontractor:

Cimentalia, Geocisa, Rodio-Kronsa, Pilotes Posada, Amilibia.

Precast slab subcontractor:

Precon 


\section{References}

[1] F. Millanes, L. Matute, M. Ortega, D. Martínez, E. Bordó, Desarrollo de soluciones mixtas y metálicas para viaductos de las líneas de alta velocidad españolas, Hormigón Acero 62 (259) (2011) 7-27.

[2] O. Schwarz, W. Zellner, R. Saul, Three bridges with the longest spans for the new high-speed railway lines, SEI 2 (2) (1992) 87-92.

[3] F. Millanes, L. Matute, M. Ortega, C. Gordo, El viaducto sobre el río Ulla en el Eje Atlántico de Alta Velocidad: una celosía mixta de $240 \mathrm{~m}$ de luz, in: V Congreso ACHE, Barcelona, 2011.

[4] S. López, I. Muñoz, F. Tarquis, F. Millanes, M. Ortega, R. Estévez, et al., Ejecución de las cimentaciones del viaducto del río Ulla en la ría de Arosa, in: V Congreso ACHE, Barcelona, 2011.

[5] R.A. Estévez, F. Millanes, M. Ortega, A. Ferrandis, Nuevos planteamientos en el control de la ejecución de puentes metálicos: el viaducto mixto sobre el río Ulla, in: V Congreso ACHE, Barcelona, 2011.

[6] F. Millanes, M. Ortega, P. Atanasio, J.L. Mansilla, D. Mouriño, Concepción de los detalles de la estructura metálica del viaducto del río Ulla, in: VI Congreso ACHE, Madrid, 2014.
[7] AENOR AEN/CTN 140 (2008): Eurocódigo 3 "Proyecto de estructuras de acero". Parte 1-9: "Fatiga”. UNE-EN 1993-1-9.

[8] F. Millanes, J. Pascual, M. Ortega, Viaducto 'Arroyo las Piedras'. Primer viaducto mixto de las Líneas de Alta Velocidad Españolas, Hormigón Acero (243) (2007) 5-38.

[9] F. Millanes, E. Bordó, J. Martín, J.L. Mansilla, Viaducto de Archidona (L.A.V. Antequera-Granada), $3.150 \mathrm{~m}$ de dintel mixto sin juntas de dilatación, Hormigón Acero 270 (2013) 7-32.

[10] S. López, F. Tarquis, D. Mouriño, J. Sanchez, R.A. Estévez, M. Ortega, Ejecución de los vanos de acceso del viaducto del río Ulla de la margen izquierda mediante lanzamiento, in: VI Congreso ACHE, Madrid, 2014.

[11] D. Mouriño, S. López, J. Sanchez, F. Tarquis, R.A. Estévez, M. Ortega, Izado de los vanos de acceso de la margen derecha del viaducto del río Ulla, in: VI Congreso ACHE, Madrid, 2014.

[12] M. Ortega, J.A. Becerra, R.A. Estévez, P. Chico, H. Corres, J. Sanchez, Ejecución de los vanos de canto variable por avance en voladizos sucesivos del Viaducto del río Ulla, in: VI Congreso ACHE, Madrid, 2014. 